\title{
Short-time transport properties in dense suspensions: from neutral to charge-stabilized colloidal spheres
}

\author{
Adolfo J. Banchio \\ CONICET and FaMAF, \\ Universidad Nacional de Córdoba, Ciudad Universitaria, X5000HUA Córdoba, Argentina \\ and \\ Gerhard Nägele \\ Institut für Festkörperforschung, Forschungszentrum Jülich, D-52425 Jülich, Germany
}

(accepted for publication in The Journal of Chemical Physics, February 2008)

${ }^{*}$ Corresponding author: Adolfo J. Banchio, electronic mail: banchio@famaf.unc.edu.ar 


\begin{abstract}
We present a detailed study of short-time dynamic properties in concentrated suspensions of charge-stabilized and of neutral colloidal spheres. The particles in many of these systems are subject to significant many-body hydrodynamic interactions. A recently developed accelerated Stokesian Dynamics (ASD) simulation method is used to calculate hydrodynamic functions, wavenumber-dependent collective diffusion coefficients, self-diffusion and sedimentation coefficients, and high-frequency limiting viscosities. The dynamic properties are discussed in dependence on the particle concentration and salt content. Our ASD simulation results are compared with existing theoretical predictions, notably those of the renormalized density fluctuations expansion method of Beenakker and Mazur, and earlier simulation data on hard spheres. The range of applicability, and the accuracy of various theoretical expressions for short-time properties, are explored through comparison with the simulation data. We analyze in particular the validity of generalized StokesEinstein relations relating short-time diffusion properties to the high-frequency limiting viscosity, and we point to the distinctly different behavior of de-ionized charge-stabilized systems in comparison to hard spheres.
\end{abstract}

PACS: $87.15 . V v$ - Diffusion

83.20.Jp - Computer simulation

82.70.Dd - Colloids 


\section{INTRODUCTION}

This paper is concerned with the calculation of transport properties characterizing the short-time dynamics of suspensions of charge-stabilized and electrically neutral colloidal spheres.

The dynamics of model dispersions of spherical particles has been the subject of continuing research both in experiment and theory (see [1-4]), motivated by the aim to understand it on a microscopic basis. Experimentally well-studied examples comprise PMMA (i.e., plexiglass) spheres immersed in an apolar organic solvent [5], aqueous suspensions of highly charged polystyrene latex spheres [2], and less strongly charged, nano-sized particle systems such as globular apoferritin proteins [6] and spherical microemulsion micelles in water [7]. Charge-stabilized colloidal dispersions, in particular, occur ubiquitously in chemical, environmental and food industry, and in many biological systems. The investigation of the dynamics in model systems of colloidal spheres is not only interesting in its own right, but might help additionally to improve our insights into transport properties of more complex colloidal or macromolecular particles relevant to industry and biology.

Due to the large size and mass disparity between colloidal particles and solvent molecules, there is a separation of time scales between the slow positional changes of the colloidal particles, and the fast relaxation of their momenta into the Maxwellian equilibrium. The momentum relaxation of the particles is characterized by the time $\tau_{B}=m /\left(6 \pi \eta_{0} a\right)$, where $m$ and $a$ are, respectively, the mass and radius of a suspended colloidal sphere, and $\eta_{0}$ is the solvent shear viscosity. The momentum relaxation time is of the same order of magnitude as the viscous relaxation time, $\tau_{\eta}=a^{2} \rho_{s} / \eta_{0}$, where $\rho_{s}$ is the solvent mass density. The time $\tau_{\eta}$ characterizes the time scale up to which solvent inertia matters. For times $t \gg \tau_{B} \sim \tau_{\eta}$, the particles and solvent move quasi-inertia-free. This characterizes the so-called Brownian dynamics time regime, wherein the stochastic time evolution of the colloidal spheres obeys a pure configuration space description as quantified by the many-particle Smoluchowski equation or the positional many-particle Langevin equation, in conjunction with the creeping flow equation describing the stationary solvent flow $[3,4]$.

In the Brownian dynamics regime, one distinguishes the short-time regime, with $\tau_{B} \ll t \ll \tau_{I}$, from the long-time regime where $t \gg \tau_{I}$. The interaction time $\tau_{I}$ can be usually estimated by $a^{2} / D_{0}$, where $D_{0}=k_{B} T /\left(6 \pi \eta_{0} a\right)$ is the translational diffusion coefficient at infinite dilution. It provides a rough estimate of the time span when direct particle interactions become important. For long times $t \gg \tau_{I}$, the motion of particles is diffusive due to the incessant bombardment by the solvent molecules, 
and the interactions with other surrounding particles originating from configurational changes. Within the colloidal short-time regime explored in this paper, the particle configuration has changed so little that the slowing influence of the direct electro-steric interactions is not yet operative. However, the short-time dynamics is influenced by the solvent mediated hydrodynamic interactions (HI) which, as a salient remnant of the solvent degrees of freedom, acts quasi-instantaneously in the Brownian time regime. In unconfined suspensions of mobile particles, the HI are very long-range and decay with the interparticle separation $r$ as $1 / r$. The inherent many-body character of the HI causes challenging problems in the theory and computer simulation studies of short-time and, to an even larger extent, of longtime dynamic properties. The HI strongly influence the diffusion and rheology in dense suspensions, and they can give rise to unexpected dynamic effects such as the enhancement of self-diffusion [8-10], and the non-monotonic density dependence [11] of long-time self-diffusion in low-salinity chargestabilized suspensions. Long-time diffusion transport quantities are in general smaller than the corresponding short-time quantities, since the dynamic caging of particles by neighboring ones, operative at longer times $\left(t \geq \tau_{I}\right)$, has a slowing influence on the dynamics. Short-time properties, on the other hand, are not subject to the dynamic caging effect since the cage has hardly changed for $t \ll \tau_{I}$. Therefore, short-time properties are more simply expressed in terms of equilibrium averages, and the direct interactions enter only indirectly through their influence on the equilibrium microstructure.

In this work, we study the short-time dynamics of dense, in the sense of strongly correlated, suspensions of charge-stabilized and neutral colloidal particles with strong (and many-body) hydrodynamic interactions. Charged colloidal particles are described on the basis of the one-component macroion fluid (OMF) model of dressed spherical macroions that interact, for non-overlap distances, by an effective screened Coulomb potential of DLVO-type. We are not concerned here with the ongoing discussion on how this state-dependent pair potential can be justified on the basis of more fundamental models of charged colloids, where the microions causing electrostatic screening are treated on equal footing with the colloids, and how the effective macroion charge, $Z e$, and the effective electrostic screening parameter, $\kappa$, are related to the microion parameters and the colloid density (see subsection 2.1 for a short discussion). For the purpose of this paper, the OMF suffices as a convenient and well-established model that captures the main features of charge-stabilized suspensions, and allows for a general discussion of their short-time dynamic properties. The OMF model spans the range from systems with long-range repulsive interactions when small values of $\kappa$ are used (i.e., low-salinity sus- 
pensions), to suspensions of neutral hard spheres in the limit of zero screening length or zero colloid charge. The freedom in selecting the screening parameter will allow us to explore the interplay of the long-distance and the short-distance parts of the $\mathrm{HI}$ in their influence on the colloid dynamics. The OMF model disregards electro-hydrodynamic effects caused by the dynamic response of the microion atmosphere. However, this is a fair approximation for colloidal particles much larger than the electrolyte ions $[6,11,12]$. Furthermore, attractive dispersion forces and possibly existing hydration forces are not considered in this model.

For the calculations of short-time properties of dense charge-stabilized suspensions, methods are required which account for many-body $\mathrm{HI}$ and proceed well beyond the pairwise-additive level that is valid at high dilution only. At larger densities, however, one needs to account for lubrication effects. For these reasons, we use here a novel accelerated Stokesian dynamics (ASD) simulation code for Brownian spheres, developed by Banchio and Brady [13], which accounts both for many-body HI and lubrication effects. Quite recently, this method has been extended in its applicability to chargestabilized systems modelled on the OMF level $[6,14,15]$. The ASD simulation method has been shown to describe short-time diffusion data for charged latex spheres, and nano-sized globular proteins, on a quantitative level of accuracy. The simulation data in $[6,14,15]$ are the only ones available so far for charge-stabilized suspensions with a full account of many-body HI.

Earlier applications of the non-accelerated (see, e.g., [16-19]) and accelerated Stokesian dynamics method $[13,20]$ pioneered by Brady and coworkers, have dealt with systems of neutral hard spheres. In addition, short-time properties of colloidal hard spheres have been computed in earlier simulation work based on the hydrodynamic force multipoles method [21], and on the fluctuating Lattice-Boltzmann (LB) method [5,22-26]. These two alternative simulation methods, both pioneered by Ladd in their application to colloids [27], have not been applied so far to charge-stabilized suspensions. In some earlier attempts [28-30] to include many-body HI into the standard Brownian dynamics simulation method of Ermak and McCammon, and into analytic calculations of short-time diffusion properties [31], pairwise-additive screened mobility tensors have been used which contain one or two concentrationdependent screening parameters that are adjusted empirically. However, whereas the hydrodynamic screening concept is well-founded in porous media with positionally fixed obstacles, no justification of its presence exists in fluid suspensions where all particles are mobile. Many-body HI in mobilesphere suspensions merely enlarge the effective suspension viscosity without reducing the range of 
the HI $[32,33]$. In fact, one of the first applications of the ASD simulation tool has been, in combination with experiment and theory, to show that the collective diffusion in low-salt charge-stabilized suspensions can be understood without the assumption of hydrodynamic screening [14, 15,34].

On the theoretical side, the explicit inclusion of $\mathrm{HI}$ into short-time dynamic properties has been achieved so far only up to hydrodynamic three-body terms, for hard spheres in the form of truncated virial expansions, and for charged spheres using truncated rooted cluster expansions. Numerically exact results for the translational and rotational short-time self-diffusion coefficients [35], for the shorttime collective diffusion coefficient [36], and for the high-frequency limiting shear viscosity of hard spheres [37], valid up to second and third order in the particle volume fraction, respectively, have been derived recently by Cichocki and collaborators. With regard to the rooted cluster expansion method for charge-stabilized particles truncated at the three-body level, approximate expressions for the equilibrium triplet distribution functions are required, since these are not known analytically [3840]. The most comprehensive theoretical scheme available so far to calculate short-time properties is the renormalized density fluctuations (named $\delta \gamma$ ) expansion approach of Beenakker and Mazur [32, 41-44], commonly referred to as the $\delta \gamma$ scheme. This method includes many-body HI in an approximate way through the consideration of so-called ring diagrams. Originally, it had been applied to hard spheres only, but in later work it's zeroth-order version was used also to predict short-time diffusion properties of charge-stabilized systems [3,34,45-50].

We will provide a comprehensive discussion of the range of applicability and the accuracy of the $\delta \gamma$ scheme, and of other theoretical and semi-empirical expressions describing short-time properties that are handy to use in the experimental data analysis. This is achieved through comparison with our ASD simulations over an extended concentration range. For hard spheres, we also compare with earlier simulation data obtained using the LB and force multipoles simulation methods, and SD simulations. While the present study is restricted in its scope to short-time properties, we note that these constitute an essential input in theories dealing with long-time dynamics [51-54].

The paper is organized as follows: Section 2 describes the one-component model of dressed spherical macroions, and the essentials of the ASD simulation scheme for neutral and charged colloidal spheres are explained. Furthermore, we give a summary of approximate theoretical expressions for various transport properties, whose range of validity will be explored in this work. The results of our ASD simulations of short-time properties are presented in section 3, and discussed in comparison 
with theory, experiment and already existing simulation data. In subsections $3.1-3.7$, we successively discuss results for the static structure factor, translational and rotational short-time self-diffusion coefficients, high-frequency limiting shear viscosity, short-time sedimentation coefficient, wave-number dependent collective diffusion functions, and generalized Stokes-Einstein (GSE) relations. Our conclusions are presented in section 4 .

\section{THEORETICAL BACKGROUND}

\subsection{One-component model}

In calculating static and dynamic properties of charge-stabilized colloidal spheres from computer simulations and analytic theories, we need to model the direct interaction potential, and to specify the properties of the embedding solvent. All our computer simulation and analytical calculations are based on the one-component macroion fluid (OMF) model. In this continuum model, the many-sphere potential of mean force obtained from averaging over the microionic and solvent degrees of freedom is supposed to be pairwise additive. The colloidal particles are described as uniformly charged hard spheres interacting by the effective pair potential of Derjaguin-Landau-Verwey-Overbeek (DLVO) type

$$
\frac{u(r)}{k_{B} T}=L_{B} Z^{2}\left(\frac{e^{\kappa a}}{1+\kappa a}\right)^{2} \frac{e^{-\kappa r}}{r}, \quad r>2 a .
$$

On assuming a closed system not in contact with an electrolyte reservoir, the electrostatic screening parameter, $\kappa$, appearing in this potential is given by

$$
\kappa^{2}=\frac{4 \pi L_{B}\left[n|Z|+2 n_{s}\right]}{1-\phi}=\kappa_{c i}^{2}+\kappa_{s}^{2},
$$

where $n$ is the colloid number density, $n_{s}$ is the number density of added 1-1 electrolyte, and $\phi=$ $(4 \pi / 3) n a^{3}$ is the colloid volume fraction of spheres with radius $a$. Furthermore, $Z$ is the charge on a colloid sphere in units of the elementary charge $e$, and $L_{B}=e^{2} /\left(\epsilon k_{B} T\right)$ is the Bjerrum length for a suspending fluid of dielectric constant $\epsilon$ at temperature $T$. The solvent is thus described as a structureless continuum characterized solely by $\epsilon$ and the shear viscosity $\eta_{0}$. We note that $\kappa^{2}$ comprises a contribution, $\kappa_{c i}^{2}$, due to surface-released counter-ions, which are assumed here to be monovalent, and a contribution, $\kappa_{s}^{2}$, arising from the added electrolyte (e.g. $\mathrm{NaCl}$ ). The factor $1 /(1-\phi)$ has been introduced to correct for the free volume accessible to the microions, owing to the presence of colloidal spheres [55]. It is of relevance for dense suspensions only. Eq. (1) can be derived from the linearized 
Poisson-Boltzmann theory [56] or the linear mean spherical approximation [3,57] on assuming pointlike microions and a weak colloid charge. Eq. (2) follows from a Poisson-Boltzmann spherical cell model calculation when the electrostatic potential is linearized around its volume average $[58,59]$.

For strongly charged spheres where $L_{B}|Z| / a>1, Z$ in Eq. (1) needs to be interpreted as an effective or renormalized charge number smaller than the bare one, that accounts to some extent for the non-linear counterion screening close to the colloid surface. Likewise, a renormalized value for $\kappa$ must be used. Several schemes have been developed to relate the effective $Z$ and $\kappa$ to the bare ones [59-63]. The outcome of these schemes depends to some extent on the approximation made for the (grand)-free energy functional, and on additional simplifying model assumptions (e.g., spherical cell or macroion jellium models).

We are not concerned here with the ongoing discussion on how the effective charge and screening parameters are related to their bare counterparts, and under what conditions three-body and higherorder corrections to the OMF pair potential come into play $[64,65]$. We merely use the OMF model as a well-defined and well-established model that captures essential features of charge-stabilized suspensions, allowing thus for a general study of their short-time dynamic properties.

In analytic calculations of short-time properties, the key input required is the colloidal static structure factor, $S(q)$, in dependence on the scattering wavenumber $q$. It is related to the radial distribution function by [66]

$$
g(r)=1+\frac{1}{2 \pi^{2} n r} \int_{0}^{\infty} d q q \sin (q r)[S(q)-1],
$$

where $g(r)$ quantifies the conditional probability of finding the center of a colloid sphere at separation $r$ from another one. To calculate $S(q)$ and $g(r)$ based on the OMF pair potential with $\kappa$ determined by Eq. (2), we have solved the Ornstein-Zernike integral equation using the well-established RogersYoung (RY) and rescaled mean spherical approximation (RMSA) schemes [3,67-69]. The RY scheme is known for its excellent structure factor predictions within the OMF model (see subsection 3.1). The RMSA results for $S(q)$ are in most cases nearly identical to the RY predictions, provided a somewhat different value of $Z$ is used. For neutral hard spheres, the RMSA becomes identical to the PercusYevick (PY) approximation. RMSA calculations, in particular, are very fast and can be efficiently used when extensive structure factor scans are needed over a wide parameter range. 


\subsection{Short-time dynamic quantities}

In the following, we summarize salient colloidal short-time expressions. Numerical results for these expressions will be discussed in section 3.

In photon correlation spectroscopy and in neutron spin echo experiments on colloidal spheres, the dynamic structure factor,

$$
S(q, t)=\left\langle\frac{1}{N} \sum_{l, j=1}^{N} \exp \left\{i \mathbf{q} \cdot\left[\mathbf{R}_{l}(0)-\mathbf{R}_{j}(t)\right]\right\}\right\rangle .
$$

is probed. In this expression, $N$ is the number of spheres in the scattering volume, $\mathbf{R}_{j}(t)$ is the vector pointing to the center of the $j$-th colloidal sphere at time $t, \mathbf{q}$ is the scattering wave vector, and $\langle\cdots\rangle$ denotes an equilibrium ensemble average. At short correlation times where $\tau_{B} \ll t \ll \tau_{I}, S(q, t)$ decays exponentially according to $[2,3]$

$$
\frac{S(q, t)}{S(q)} \approx \exp \left[-q^{2} D(q) t\right]
$$

with a wavenumber-dependent short-time diffusion function $D(q)$. An application of the generalized Smoluchowski equation leads to the well-known expression [3]

$$
D(q)=D_{0} \frac{H(q)}{S(q)}
$$

relating $D(q)$ to the hydrodynamic function $H(q)$, and to the static structure factor $S(q)=S(q, t=0)$. The microscopic expression for $H(q)$ is given by [70]

$$
H(q)=\left\langle\frac{k_{B} T}{N D_{0}} \sum_{l, j=1}^{N} \hat{\mathbf{q}} \cdot \mu\left(\mathbf{R}^{N}\right)_{l j} \cdot \hat{\mathbf{q}} \exp \left\{i \mathbf{q} \cdot\left[\mathbf{R}_{l}-\mathbf{R}_{j}\right]\right\}\right\rangle .
$$

Here, $\hat{\mathbf{q}}$ is the unit vector in the direction of $\mathbf{q}$, and the $\mu\left(\mathbf{R}^{N}\right)_{l j}$ are the hydrodynamic mobility tensors relating a force on sphere $j$ to the velocity of sphere $l$. The mobilities depend in general on the positions, $\mathbf{R}^{N}$, of all particles which makes an analytic calculation of $H(q)$ intractable, unless approximations are introduced.

The positive-valued function $H(q)$ contains the influence of the HI on the short-time diffusion. It can be expressed as the sum of a $q$-independent self-part, and a $q$-dependent distinct-part according to

$$
H(q)=\frac{D_{s}}{D_{0}}+H_{d}(q)
$$


where $D_{s}$ is the short-time translational self-diffusion coefficient. The coefficient $D_{s}$ gives the initial slope of the particle mean squared displacement $W(t)$, defined in three dimensions by

$$
W(t)=\frac{1}{6}\left\langle[\mathbf{R}(t)-\mathbf{R}(0)]^{2}\right\rangle .
$$

In the large- $q$ limit, the distinct part vanishes and $H(q)$ becomes equal to $D_{s} / D_{0}$. Without HI, $H(q)$ is equal to one for all values of $q$. Any variation in its dependence on the scattering wave number is thus a hallmark of the influence of HI. In the experiment, $H(q)$ is determined by short-time dynamic measurement of $D(q)$ in combination with a static measurement of $S(q)[15,71]$. In physical terms, $H(q)$ can be interpreted as the (reduced) short-time generalized mean sedimentation velocity in a homogeneous suspension subject to weak force field collinear with $\mathbf{q}$ and oscillating spatially as $\cos (\mathbf{q}$. r). As a consequence,

$$
\lim _{q \rightarrow 0} H(q)=\frac{U_{s}}{U_{0}}
$$

is equal to the concentration-dependent short-time sedimentation velocity, $U_{s}(\phi)$, of a slowly settling suspension of spheres measured relative to the sedimentation velocity, $U_{0}$, at infinite dilution. Thus the long wavelength limit of $H(q)$ can be determined in an alternative way by means of macroscopic sedimentation experiments [72].

Furthermore, for $q a \ll 1, D(q)$ reduces to the short-time collective or gradient diffusion coefficient, $D_{c}$, given by

$$
D_{c}=\frac{D_{0}}{S(0)} \frac{U_{s}}{U_{0}}
$$

The collective diffusion inherits its concentration dependence from $U_{s}(\phi)$ and the thermodynamic factor $S(0):=\lim _{q \rightarrow 0} S(q)$. At non-zero concentration, $U_{s}(\phi)<U_{0}$, and $D_{c}(\phi)$ is different from $D_{0}$. Likewise, $D_{s}(\phi>0)$ is smaller than $D_{0}$ in the presence of HI. Because of configurational changes taking place for times $t>\tau_{I}$, long-time transport properties are smaller than the short-time ones. A case in point is self-diffusion, where the long-time coefficient $D_{l}$ can be substantially smaller than $D_{s}$. By contrast, sedimentation is hardly affected by dynamic caging so that the long-time sedimentation velocity $U_{l}$ in a fluid-like suspension is only slightly smaller than $U_{s}$. The difference is less than $6 \%$ even when a concentrated hard-sphere suspension is considered [73]. This is explained by the fact that the equilibrium microstructure of identical spheres settling under a constant force is only slightly perturbed by many-body HI, and not affected at all by the pairwise additive two-body part of the HI. 
Up to this point only translational diffusion has been discussed. For optically anisotropic spheres, characterized in their orientation by a unit vector $\hat{\mathbf{u}}(t)$, rotational dynamics can be probed by experimental techniques sensitive to their orientation $[40,74]$. In a depolarized dynamic light scattering measurement, e.g., the rotational self-dynamic correlation function [39],

$$
S_{r}(t)=\left\langle P_{2}(\hat{\mathbf{u}}(t) \cdot \hat{\mathbf{u}}(0))\right\rangle,
$$

is probed, with $P_{2}$ denoting the Legendre polynomial of second order. At short times, $S_{r}(t)$ decays exponentially according to

$$
S_{r}(t) \approx \exp \left\{-6 D_{r} t\right\}
$$

where the short-time rotational self-diffusion coefficient $D_{r}$ is the rotational analogue of $D_{s}$. At finite colloid concentration, rotational diffusion is slowed by the HI so that $D_{r}(\phi)<D_{0}^{r}$. Here, $D_{0}^{r}=$ $k_{B} T /\left(8 \pi \eta_{0} a^{3}\right)$ is the rotational diffusion coefficient at infinite dilution.

Many attempts have been made in the past to identify generalized Stokes-Einstein (GSE) relations between diffusional and viscoelastic suspension properties. These relations are of interest also from an experimental point of view since, provided they are valid, rheological properties are probed more easily and for smaller probe volumes using scattering techniques. Out of several GSE proposals, we will explore the following three short-time relations, namely $[52,76]$

$$
D_{s}(\phi)=\frac{k_{B} T}{6 \pi \eta_{\infty}(\phi) a},
$$

and $[40,77]$

$$
D_{r}(\phi)=\frac{k_{B} T}{8 \pi \eta_{\infty}(\phi) a^{3}}
$$

and $[52,77]$

$$
D\left(q_{m} ; \phi\right)=\frac{k_{B} T}{6 \pi \eta_{\infty}(\phi) a},
$$

which relate the high-frequency limiting viscosity $\eta_{\infty}$ to $D_{s}, D_{r}$, and to the short-time diffusion function $D(q)$, respectively, the latter evaluated at the wave number $q_{m}$ where $S(q)$ and $H(q)$ attain their maximum. In what follows, we will refer to $D\left(q_{m}\right)$ as the (short-time) cage diffusion coefficient, since $2 \pi / q_{m}$ characterizes the radius of the next-neighbor shell of particles. The short-time viscosity $\eta_{\infty}$ reflects the bulk dissipation in a suspension subject to a high-frequency, low amplitude shear oscillation of circular frequency $\omega$ with $\left(\tau_{I}\right)^{-1} \ll \omega \ll\left(\tau_{\eta}\right)^{-1}$. We will expose these relations to a stringent test using our ASD simulation data for $\eta_{\infty}(\phi)$, in combination with simulation results for $D_{s}, D_{r}$, and $H\left(q_{m}\right)$. 


\subsection{Computational methods}

The main tool for calculating the short-time quantities discussed previously will be accelerated Stokesian dynamics simulations, using a code for Brownian particles developed by Banchio and Brady [13], and extended by us to charged spheres interacting via the OMF pair potential of Eqs. (1) and (2). This code enables us to simulate the short-time properties of a larger number of spheres, typically up to 1000 placed in a periodically replicated simulation box, which leads to an improved statistics. The details of this simulation method have been explained in Ref. [13] and will not be repeated here. To speed up the computation of short-time quantities such as $H(q)$, which require for their computation a single-time equilibrium averaging only, we have generated a set of equilibrium configurations using a Monte-Carlo (MC) simulation code in the case of charge-stabilized spheres, and a Molecular Dynamics (MD) simulation code for neutral hard spheres. The many-body HI have been computed using the ASD scheme. To correct for finite-size effects arising from the periodic boundary conditions, the ASD simulations have been extrapolated to the thermodynamic limiting form of $H(q)$ by using the finite-size scaling correction

$$
H(q)=H_{N}(q)+1.76 S(q) \frac{\eta_{0}}{\eta_{\infty}(\phi)}(\phi / N)^{1 / 3},
$$

which, for $q \rightarrow \infty$, includes the finite-size correction for $D_{s}$ as a special case. This correction formula was initially proposed by Ladd in the framework of LB simulations of colloidal hard spheres [5,21,22]. The finite-size scaling form used to extrapolate to the $H(q)$ of an infinite system requires thus to compute the high-frequency limiting viscosity $\eta_{\infty}(\phi)$. Whereas empirical expressions for $\eta_{\infty}(\phi)$ are available in the case of hard spheres (see the following subsection), simulations of $\eta_{\infty}$ are required in general for charge-stabilized spheres. In section 3, we will show that the simulation data obtained for various $N$ collapse neatly on a single master curve once Eq. (17) has been used. The resulting master curve is identified with the finite-size corrected form of $H(q)$.

The only analytic method available to date allowing to predict the $H(q)$ of dense suspensions of neutral or charge-stabilized spheres, is the (zeroth order) renormalized density fluctuation expansion method of Beenakker and Mazur [32]. This so-called $\delta \gamma$ method is based on a partial resummation of the many-body HI contributions. According to this scheme, $H(q)$ is obtained to leading order in the renormalized density fluctuations from $[32,45]$

$$
H_{d}(q)=\frac{3}{2 \pi} \int_{0}^{\infty} d(a k)\left(\frac{\sin (a k)}{a k}\right)^{2}\left[1+\phi S_{\gamma_{0}}(a k)\right]^{-1} \times \int_{-1}^{1} d x\left(1-x^{2}\right)[S(|\mathbf{q}-\mathbf{k}|)-1],
$$


and

$$
\frac{D_{s}(\phi)}{D_{0}}=\frac{2}{\pi} \int_{0}^{\infty} d t\left(\frac{\sin t}{t}\right)^{2}\left[1+\phi S_{\gamma_{0}}(t)\right]^{-1}
$$

where $x$ is the cosine of the angle extended by the wave vectors $\mathbf{q}$ and $\mathbf{k}$, and $S_{\gamma_{0}}(t)$ is a known function independent of the particle correlations and given in Refs. [32,45]. The only input required is the static structure factor $S(q)$, which we calculate using the RY and RMSA integral equation schemes. As can be noted from Eq. (18), $S(q)$ enters only into the distinct part of $H(q)$ since to lowest order in the renormalized density fluctuations expansion, the self-part is independent of $S(q)$. For charged spheres, the short-time self-diffusion coefficient is thus more roughly approximated by the value for neutral hard spheres at the same $\phi$, independent of the sphere charge and screening parameter. To include the actual pair correlations into the calculation of $D_{s}$ requires to go one step further in the fluctuating density expansion, which severely complicates the scheme. Yet, from comparing the $\delta \gamma$ scheme predictions with ASD simulations and experimental data of charge-stabilized systems, we find that $H_{d}(q)$ is in general well captured by Eq. (18). This observation allows us to improve the $\delta \gamma$ scheme through replacing the $\delta \gamma-D_{s}$ by the simulation prediction. The $\delta \gamma-H(q)$ is then shifted upwards by a small to moderately large amount, owing to the fact that the $D_{s}$ of charged spheres is larger than for neutral ones $[3,75]$. Even without a correction for $D_{s}$, the $\delta \gamma$ scheme remains useful in predicting, on a semi-quantitative level, general trends in the behavior of $H(q)$.

\subsection{Analytic short-time expressions}

Virial expansions for short-time properties of colloidal hard spheres are meanwhile available up to the hydrodynamic three-body level. These results are summarized in the following and compared to semiempirical expressions that apply to volume fractions up to $\phi=0.5$. The equilibrium microstructure of charge-stabilized particles depends on several system parameters including $\phi, \kappa a$ and $L_{B}|Z|^{2} / a$. Therefore, for these systems, general analytic expressions describing the $\phi$ dependence of short-time properties are not available, except for suspensions of strongly charged spheres at low salinity, where screening is dominated by surface-released counterions. In these systems, part of the transport coefficients reveal a concentration dependence of fractional order. 


\subsubsection{Hard spheres}

The following truncated virial expansion results for hard spheres have been derived by Cichocki and collaborators [35-37]

$$
\begin{aligned}
& D_{s} / D_{0}=1-1.832 \phi-0.219 \phi^{2}+\mathcal{O}\left(\phi^{3}\right) \\
& D_{r} / D_{0}^{r}=1-0.631 \phi-0.726 \phi^{2}+\mathcal{O}\left(\phi^{3}\right) \\
& U_{s} / U_{0}=1-6.546 \phi+21.918 \phi^{2}+\mathcal{O}\left(\phi^{3}\right) \\
& \eta_{\infty} / \eta_{0}=1+2.5 \phi+5.0023 \phi^{2}+9.09 \phi^{3}+\mathcal{O}\left(\phi^{4}\right) .
\end{aligned}
$$

These results fully account for HI up to the three-body level, and they include lubrication corrections. The virial expression for $\eta_{\infty}$ contains a term proportional to $\phi^{3}$, since the viscosity is influenced by particle correlations only when at least terms of quadratic order in $\phi$ are considered. From the virial expressions for $D_{s}$ and $\eta_{\infty}$, we note that the GSE expressions in Eqs. (14) and (15) are violated to a certain extent to quadratic order in $\phi$.

Eq. (22) for the sedimentation coefficient divided by the expansion, $S(0)=1-8 \phi+34 \phi^{2}+$ $\mathcal{O}\left(\phi^{3}\right)$, for the reduced osmotic compressibility of hard spheres, gives the 2nd-order virial result

$$
\frac{D_{c}}{D_{0}}=1+1.454 \phi-0.45 \phi^{2}+\mathcal{O}\left(\phi^{3}\right)
$$

for the collective diffusion coefficient valid up to quadratic order in $\phi$. This expression describes a weak initial increase in $D_{c}$ when $\phi$ is increased. From the simulation results discussed in section 3) we will see that this slow increase of $D_{c}$ extends up to the concentration $\phi \approx 0.5$ where the system starts to freeze. For charged spheres, the initial of $D_{c}(\phi)$ at small $\phi$ is typically far steeper. Moreover, on further increasing $\phi, D_{c}$ passes through a maximum. This maximum is due to the competing influences of $U_{s}$ and $S(0)$. At small $\phi, S(0)$, decreases faster in $\phi$ than the sedimentation coefficient, whereas this trend is reversed at larger $\phi[6,45,69]$ where the slowing influence of the HI becomes strong. Although the OMF potential used in this simulation study is purely repulsive, a few comments on the influence of attractive interactions are in order here. For particles subject to a significant short-range attractive interaction part such as lysozyme globules [78], $D_{c}(\phi)$ can decrease with increasing $\phi$ and attain values well below $D_{0}$ [79]. Attractive forces tend to enlarge both the sedimentation velocity and the compressibility, but the raise in the compressibility is larger and causes $D_{c}$ to decrease with increasing $\phi[79]$. Attractive forces tend to increase the probability of neighboring particles to be 
found at close proximity, causing thus a decrease in the short-time self-diffusion coefficient through the stronger passive hindrance of the self-motion by neighboring particles [80]. This corresponds to an attraction-induced increase in the high-frequency limiting viscosity [81].

A few semi-empirical expressions for short-time properties of hard spheres are frequently used in the literature. Here we quote a formula for the self-diffusion coefficient proposed by Lionberger and Russel [82]

$$
\frac{D_{s}}{D_{0}}=(1-1.56 \phi)(1-0.27 \phi)
$$

This expression conforms overall well, up to $\phi=0.3$, with the truncated virial expansion result for $D_{s}$ in Eq. (20), it reduces to the correct low-density limit $1-1.83 \phi+\mathcal{O}\left(\phi^{2}\right)$, and it diverges at the value $\phi_{\text {rcp }} \approx 0.64$ where random close packing occurs. For volume fractions exceeding 0.3 , the second order virial expression for $D_{s}$ ceases to be applicable [69].

The bulk of experimental data on $\eta_{\infty}$ conforms overall well with another empirical expression of Lionberger and Russel [82],

$$
\frac{\eta_{\infty}}{\eta_{0}}=\frac{1+1.5 \phi\left(1+\phi-0.189 \phi^{2}\right)}{1-\phi\left(1+\phi-0.189 \phi^{2}\right)} .
$$

This formula has built in the exact Einstein limiting law at very small concentrations, and it diverges for random close packing. The force multipole simulation data of $\eta_{\infty}$ obtained by Ladd are well fitted by the formula [21]

$$
\frac{\eta_{\infty}}{\eta_{0}}=\frac{1+1.5 \phi(1+S(\phi))}{1-\phi(1+S(\phi))}
$$

with $S(\phi)=\phi+\phi^{2}-2.3 \phi^{3}$. In section 3 we will show that our ASD simulation data for the shorttime viscosity conform to the simulation results of Ladd, and that the third-order virial expression for $\eta_{\infty}$ in Eq. (23) is applicable up to $\phi \approx 0.25$. Instead of showing a comparison with experimental data for $\eta_{\infty}$, we will merely compare the simulation data to the outcome of Eq. (26), used as a representative of the experimental data. For a Saito-like expression for $\eta_{\infty}$ based on two-body HI see [83].

The principal peak value, $H\left(q_{m}\right)$, of $H(q)$ occurs practically at the same wave number, $q_{m}$, where $S(q)$ attains its largest value. The peak height is well represented by the linear form [52],

$$
H\left(q_{m}\right)=1-1.35 \phi
$$

which is valid for all concentrations up to the freezing concentration of hard spheres. This remarkable expression should be compared to the prediction of the $\delta \gamma$ scheme, with PY input for $S(q)$, which is 
well represented, for densities $\phi<0.45$, by the quadratic form [48]

$$
H\left(q_{m}\right)=1-2.03 \phi+1.94 \phi^{2}
$$

As we will show, the $\delta \gamma$ scheme underestimates the correct peak height described by Eq. (28). For $\phi<0.35, H\left(q_{m}\right)$ is moderately underestimated, but it is strongly overestimated for $\phi>0.4$.

On dividing Eq. (28) for $H\left(q_{m}\right)$ by an empirical expression for the peak height of the Verlet-Weiss corrected PY-S $(q)$, that is of good accuracy up to $\phi \leq 0.5$, namely by [84]

$$
S\left(q_{m}\right)=1+0.644 \phi g_{\mathrm{CS}}\left(2 a^{+}\right),
$$

where

$$
g_{\mathrm{CS}}\left(2 a^{+}\right)=\frac{(1-0.5 \phi)}{(1-\phi)^{3}},
$$

we obtain an analytic expression for $D\left(q_{m}\right)$ which is in perfect agreement with experimental data on hard spheres [85], LB simulations of Behrend [5], and with our ASD simulation results. This expression for $D\left(q_{m}\right)$, can be used to scrutinize for hard spheres the validity of the GSE relation (16) relating $\eta_{\infty}$ to $D\left(q_{m}\right)$. In Eq. (31), $g_{\mathrm{CS}}\left(2 a^{+}\right)$is the Carnahan-Starling contact value for the radial distribution function of hard spheres which is found to be in good agreement with computer simulations $[66,86]$. According to Eq. $(30), S\left(q_{m} ; \phi=0.494\right)=2.85$, in agreement with the empirical Hansen-Verlet rule for the onset of freezing [66].

Simulation data for the collective diffusion coefficient, $D_{c}(\phi)$, are readily obtained from the force multipole [21] and the LB simulation [5] data for $U_{s} / U_{0}$, on dividing these data through $S(0)$ as described by the rather accurate Carnahan-Starling expression,

$$
S_{\mathrm{CS}}(0)=\frac{(1-\phi)^{4}}{(1+2 \phi)^{2}+\phi^{3}(\phi-4)} .
$$

The so-obtained simulation data can be compared to the second order virial expression for $D_{c}$. This comparison shows that the latter is applicable up to surprisingly large concentrations $\phi \leq 0.4$. At larger values $\phi>0.4, D_{c}$ is underestimated. Note here that $S(0)$ calculated both in the CS and PY approximations remains exact up to quadratic order in $\phi$. For $\phi=0.5$, the Carnahan-Starling predicted value for $S(0)$ exceeds the PY value by only $12 \%$.

\subsubsection{Charge-stabilized spheres}

In contrast to hard spheres, a regular virial expansion is not applicable to determine the diffusion transport coefficient of strongly charged spheres in low salinity suspensions [3]. 
Instead, for de-ionized systems, the following non-linear concentration dependencies have been predicted by theory $[3,46,47,87]$

$$
\begin{aligned}
& D_{s} / D_{0}=1-a_{t} \phi^{4 / 3} \\
& D_{r} / D_{0}^{r}=1-a_{r} \phi^{2} \\
& U_{s} / U_{0}=1-a_{s} \phi^{1 / 3},
\end{aligned}
$$

with parameters $a_{t} \approx 2.5$ and $a_{r} \approx 1.3$ which depend only weakly on the particle charge and size. The coefficient $a_{s} \approx 1.8$ in the expression for the sedimentation coefficient is nearly constant. The present expressions for $D_{r}$ and $D_{s}$ have been confirmed experimentally $[69,75]$, and we will show that they conform with existing LB and our ASD simulation data up to $\phi \approx 0.35$ and $\phi \approx 0.1$, respectively.

The peak height of $H(q)$ in these systems is well approximated, for densities $\phi \leq 10^{-2}$, by the non-linear form [52]

$$
H\left(q_{m}\right) \approx 1+p_{m} \phi^{0.4}
$$

The coefficient $p_{m}>0$ in this expression is moderately depending on $Z$ and $\kappa a$. It is larger for more strongly structured suspensions characterized by a higher peak in $S(q)$. The exponent 0.4 , however, is independent of the system parameters, as long as the particle charge is large enough that the physical hard core of the spheres remains totally masked by a sufficiently strong and long-range electrostatic repulsion. The values of the exponents in the short-time expressions of Eqs. (33-36) can be attributed essentially to the fact that, in de-ionized suspensions of strongly charge spheres, the location, $r_{m}=$ $2 \pi / q_{m}$, characterizing the location of the principal peak in $g(r)$ that characterizes the location of the next-neighbor shell coincides, within $5 \%$ of accuracy (see [87] and Fig. 5), with the geometric mean distance $\bar{r}=n^{-1 / 3}=a(4 \pi / 3 \phi)^{1 / 3}$ of two spheres.

To calculate $H(q)$ for very dilute de-ionized suspensions, it suffices to account for the leadingorder far-field form of the hydrodynamic mobilities only, which amounts to the neglect of hydrodynamic reflections with constant force densities on the sphere surfaces (so-called Rotne-Prager approximation of the HI). Then a simple analytic expression is obtained $[3,88]$,

$$
H_{\mathrm{RP}}(y)=1-15 \phi \frac{j_{1}(y)}{y}+18 \phi \int_{1}^{\infty} d x x[g(x)-1]\left(j_{0}(x y)-\frac{j_{1}(x y)}{x y}+\frac{j_{2}(x y)}{6 x^{2}}\right),
$$

where $y=2 q a, x=r /(2 a)$, and $j_{n}$ is the spherical Bessel function of order $n$. Experimental results for the hydrodynamic functions of highly dilute dispersions of strongly charged particles are well described by this expression [90]. However, Eq. (37) necessarily fails for larger particle densities, when 
near-field $\mathrm{HI}$ contributions come into play [3]. It is then necessary to resort to $\delta \gamma$ scheme calculations or to simulations of $H(q)$. In Rotne-Prager (RP) approximation, the short-time sedimentation coefficient is given by,

$$
U_{s} / U_{0}=1+\phi+12 \phi \int_{0}^{\infty} d x x h(x)=1+\phi+12 \phi \widetilde{H}(z \rightarrow 0),
$$

where $\widetilde{H}(z)$ is the Laplace transform of $x h(x)$ and $h(x)=g(x)-1$. Using the analytic PercusYevick expression for the function $\widetilde{H}(z)$ of hard spheres given by Wertheim [91], the RP sedimentation coefficient is

$$
U_{s} / U_{0}=\frac{(1-\phi)^{3}}{1+2 \phi}+\frac{1}{5} \phi^{2} \approx 1-5 \phi+\frac{66}{5} \phi^{2}+\mathcal{O}\left(\phi^{3}\right)
$$

where we have accounted for the extra term $\phi^{2} / 5$ omitted by Brady and Durlofsky [33] in their original derivation of $U_{s}$ in RP approximation. At large values of $\phi$, this correction term spoils to some extent the reasonably good agreement with the simulation data of hard spheres, that would be observed otherwise (see subsection 3.4). On first sight one might expect the RP approximation to be very poor for hard spheres. With regard to sedimentation, however, it actually captures the main effect since, as discussed thoroughly by Brady and co-workers $[16,17,33]$, the contribution of near-field HI to the sedimentation velocity, in the form of induced stresslets which are of $\mathcal{O}\left(r^{-4}\right)$ and lubrication is quite small, unless $\phi$ is so large that near-contact configurations become non-negligible.

The short-time viscosity in dilute suspensions of strongly repelling spheres with prevailing twobody HI can be computed from $[1,89]$

$$
\frac{\eta_{\infty}}{\eta_{0}}=1+\frac{5}{2} \phi(1+\phi)+\frac{15}{2} \phi^{2} \int_{2}^{\infty} d x x^{2} g(x) J(x)
$$

where the rapidly and monotonically decaying function $J(x)$ accounts for two-body HI. It is known numerically, and as an expansion in $a / r$, with long-distance asymptotic form $J(x)=(15 / 2) x^{-6}+$ $\mathcal{O}\left(x^{-8}\right)$, for $x$ defined here and in the following as $x=r / a \gg 1$. The $(5 / 2) \phi^{2}$ term arises from regularizing the integral, which is a summation over induced hydrodynamic force dipoles. The integral involving the radial distribution function is at most of order one. In dilute and deionized systems, where $r_{m} \sim \phi^{-1 / 3}$, the integral is a small quantity of $\mathcal{O}(\phi)$. For these systems, $\eta_{\infty}$ is well approximated by the single-body part in Eq. (40). If we account in Eq. (40) for the leading-order long-distance part of $J(x)$ only, then

$$
\frac{\eta_{\infty}}{\eta_{0}} \approx 1+\frac{5}{2} \phi(1+\phi)+\frac{75}{256} \phi^{2} \int_{0}^{\infty} d u u^{4} \widetilde{G}(u)
$$


where $\widetilde{G}(u)$ is the Laplace transform of $x g(x)$. This expression is handy to use, since the RMSA provides an analytic expression for $\widetilde{G}(u)$. Next, we schematically approximate $g(x)$ in Eq. (40) by the form [52]

$$
g(x) \approx(\bar{r} / a) A\left(\phi_{\mathrm{eff}}\right) \delta(x-\bar{r} / a)+\Theta(x-\bar{r} / a)
$$

consisting of a step function describing the correlation hole formed around each sphere, and a deltadistribution term describing the first maximum in $g(r)$. To determine the amplitude factor $A\left(\phi_{\text {eff }}\right)$, with $\phi_{\text {eff }}=\phi(\bar{r} / a)^{3}=\pi / 6$, we use the compressibility equation

$$
1+3 \phi_{\mathrm{eff}} \int_{0}^{\infty} d x x^{2}[g(x)-1]=\chi_{T}\left(\phi_{\mathrm{eff}}\right),
$$

where $\chi_{T}\left(\phi_{\mathrm{eff}}\right)=k_{B} T(\partial n / \partial p)_{T}$ is the reduced isothermal compressibility of hard spheres, evaluated at $\phi_{\text {eff }}$, for which we employ the Carnahan-Starling equation of state. As a result, we obtain $A\left(\phi_{\text {eff }}\right) \approx$ 0.25 , which follows also from neglecting the very small $\chi_{T}$ of deionized systems as compared to one. Using this value for $A$ leads to [52]

$$
\frac{\eta_{\infty}}{\eta_{0}} \approx 1+\frac{5}{2} \phi(1+\phi)+7.9 \phi^{3}
$$

which includes a positive-valued correction term, $7.9 \phi^{3}$, with a coefficient that is smaller than the coefficient, 9.09, of the third-order density contribution in the virial expansion of hard spheres in Eq. (23). The correction term describes the viscosity contribution arising from binary particle correlations, and we emphasize that it is of cubic order in $\phi$ since $\bar{r} \sim \phi^{-1 / 3}$ and $J(x) \sim 1 / x^{6}$ in deionized systems. As we will show, Eq. (44) is in good accord with our ASD simulation data on deionized suspensions, for the concentration range considered (see subsection 3.3).

\section{RESULTS AND DISCUSSION}

The ASD simulation and $\delta \gamma$ theory results for the colloidal short-time properties of charge-stabilized spheres shown is this section are based on the one-component macroion fluid model (OMF), with the effective pair potential given by Eqs. 1 and 2. This model contains neutral hard spheres as the limiting case of zero colloid charge or infinitely strong screening. The solvent is described as a structureless continuum of dielectric constant $\epsilon=10$ and temperature $T=298.15 \mathrm{~K}\left(25^{\circ} \mathrm{C}\right)$, with a Bjerrum length of $L_{B}=5.617 \mathrm{~nm}$. The colloidal sphere radius is selected as $a=100 \mathrm{~nm}$, and the effective colloid charge number is $Z=100$, corresponding to $L_{B}|Z| / a=5.62$ and a low-salt contact potential of 
$\beta u\left(2 a^{+}\right)=281$ valid for $\kappa a \ll 1$. Most of our simulation and theoretical results on charge-stabilized systems discussed in the following have been obtained using these interaction parameters, and it will be noted explicitly if other system parameters have been used. The selected system parameters are representative of suspensions of strongly charged colloidal spheres. We focus our discussion on deionized charge-stabilized suspensions, where all excess electrolyte ions have been removed, and on suspensions of neutral colloidal spheres. However, for a selected number of properties we will also discuss the transition from salt-free to neutral hard-sphere suspensions as the salt content is increased. The short-time properties of systems with finite amount of added salt are bounded by the values for these two limiting model systems, as we will illustrate by examples. All short-time properties are explored over an extended range of volume fractions where the suspensions show fluid-like order.

\subsection{Static structure factor}

In Fig. 1, we discuss the $q$-dependence of the static structure factor of hard spheres, for three selected volume fractions as indicated in the figure. The RY predictions of $S(q)$ are nearly coincident with the MD simulation results, and with the Verlet-Weiss corrected PY structure factor, even for the largest volume fraction considered. The uncorrected PY approximation, however, noticeably overestimates the structure factor peak height, $S\left(q_{m}\right)$, for concentrations larger than $\phi=0.4$. This can be clearly seen from Fig. 2, which shows the PY-S $\left(q_{m}\right)$ in comparison with the accurate VW-PY peak height that is well parameterized by the analytic expression in Eq. (30). Fig. 3 displays the corresponding hard-sphere radial distribution functions $g(r)$. The RY scheme is in better agreement with the MD simulation results than the PY approximation. However, it also underestimates the contact value of $g(r)$ for volume fractions near the freezing transition value. The Verlet-Weiss corrected PY scheme, on the other hand, has been designed to agree well with the simulation data.

The static structure factor of a salt-free suspension of highly charged spheres is shown in Fig. 4 for volume fractions as indicated. The RY-calculated $S(q)$, obtained using the same system parameters as in the simulation, nearly coincides with the simulation data over the whole displayed range of wave numbers. Small differences are observed in $S\left(q_{m}\right)$ only, which at larger $\phi$ is slightly underestimated by the RY scheme. The agreement between RY theory and simulation in the peak height becomes even better for systems with added electrolyte. In the figure, we do not show the $S(q)$ obtained from the linear RMSA scheme. This scheme requires usually somewhat larger values of the effective col- 


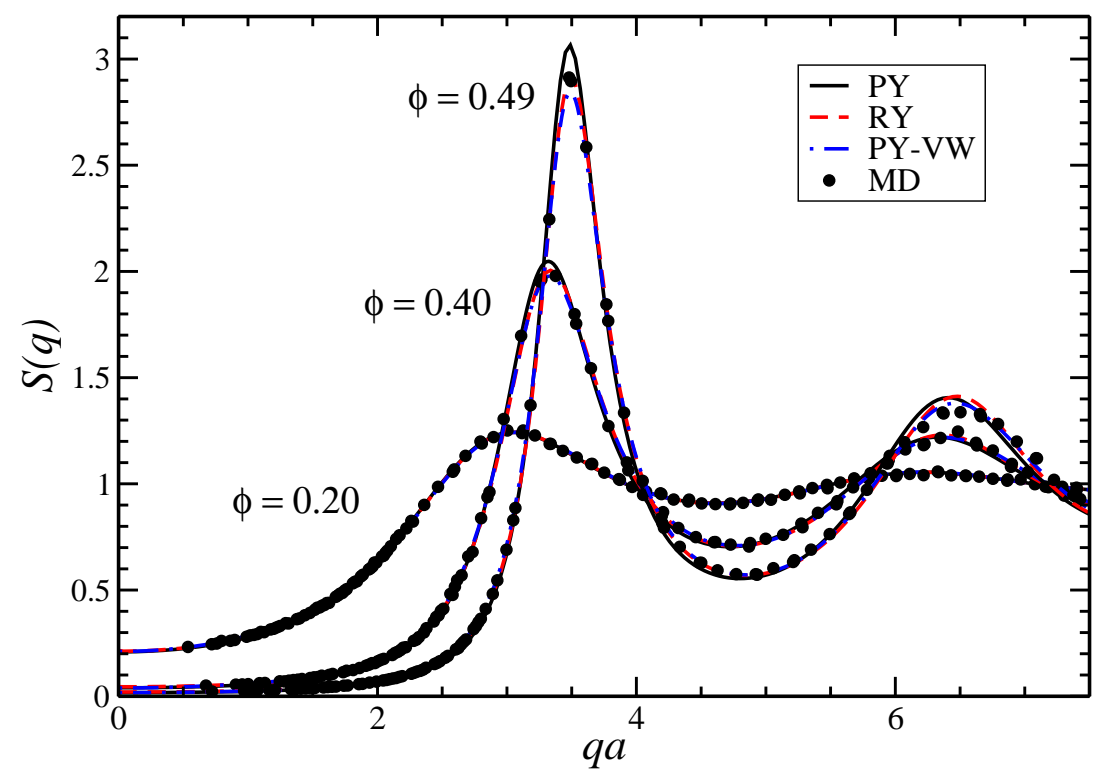

Figure 1: (Color online) Static structure factor of a hard-sphere suspension at various volume fractions $\phi$ as indicated. Comparison between MD simulation data and Rogers-Young (RY), Percus-Yevick (PY) and VerletWeiss corrected Percus-Yevick (PY-VW) integral equation schemes.

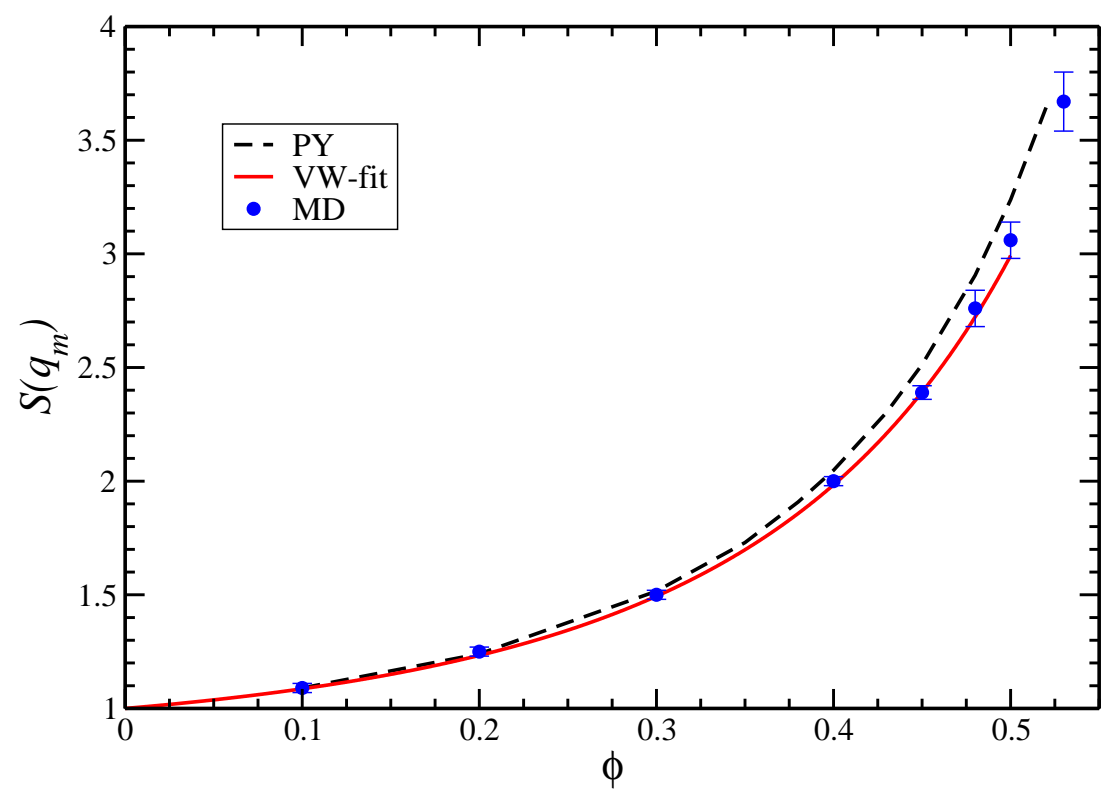

Figure 2: (Color online) Peak value, $S\left(q_{m}\right)$, of the hard-sphere static structure factor. MD simulation data (symbols) are compared with PY calculations and the PY-VW fitting formula in Eq. (30). 


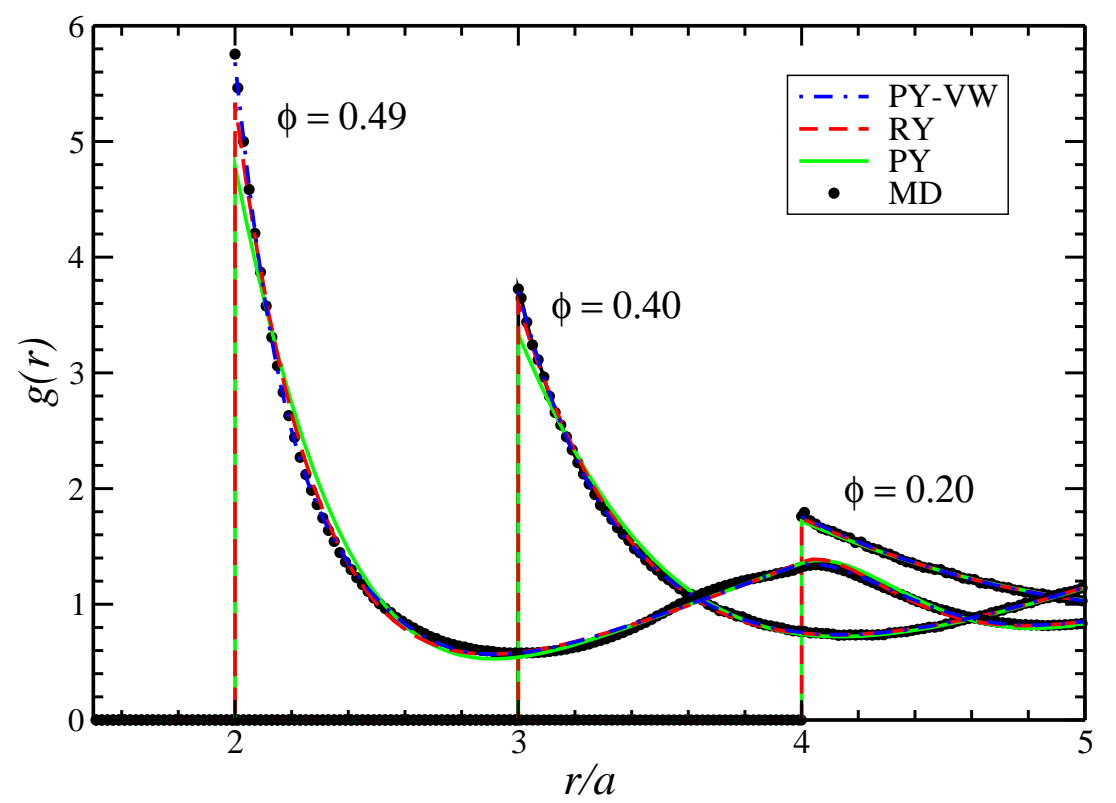

Figure 3: (Color online) Hard-sphere radial distribution function corresponding to Fig. 1. The curves for $\phi=0.40$ and 0.20 are shifted to the right by $a$ and $2 a$, respectively.

loid charge, $Z e$, to match the peak height of the simulated $S(q)$. This reflects the well-known fact that in RMSA the pair correlations are somewhat underestimated for suspensions of strongly charged particles. However, once the RMSA effective charge has been adjusted accordingly, remarkably good agreement is observed between the RMSA and RY structure factors [6]. Moreover, even for a nonadjusted effective charge, the position, $q_{m}$, of the structure factor peak is very well described by the RMSA scheme. The position, $r_{m}$, of the principal peak in the radial distribution function in these systems coincides within $5 \%$ with the average geometric distance, $\bar{r}=n^{-1 / 3}$, of two particles [87], which is here the only physically relevant static length scale. This feature has been used to derive the concentration-scaling predictions quoted in Eqs. (33-36) and (44). That the peak position, $q_{m}$, for these systems scales indeed as $2 \pi / \bar{r}=\left(6 \pi^{2} \phi\right)^{1 / 3} / a$, up to a factor of about 1.1 , can be seen from Fig. 5 which displays the RMSA prediction for $q_{m}$, in comparison with the VW-PY calculated peak position of hard spheres. The two curves tend to converge at large $\phi$ since $r_{m}$ approaches $2 a$ for increasing concentration. The red circles describe a system at $\phi=0.15$, where $n_{s}$ is increasing from $1 \times 10^{-6}$ to $1 \times 10^{-4} \mathrm{M}$ (i.e., from $\kappa a=2$ to 10 ). This illustrates the transition, for a fixed $\phi$, from a low-salt to a hard-sphere-like system. 


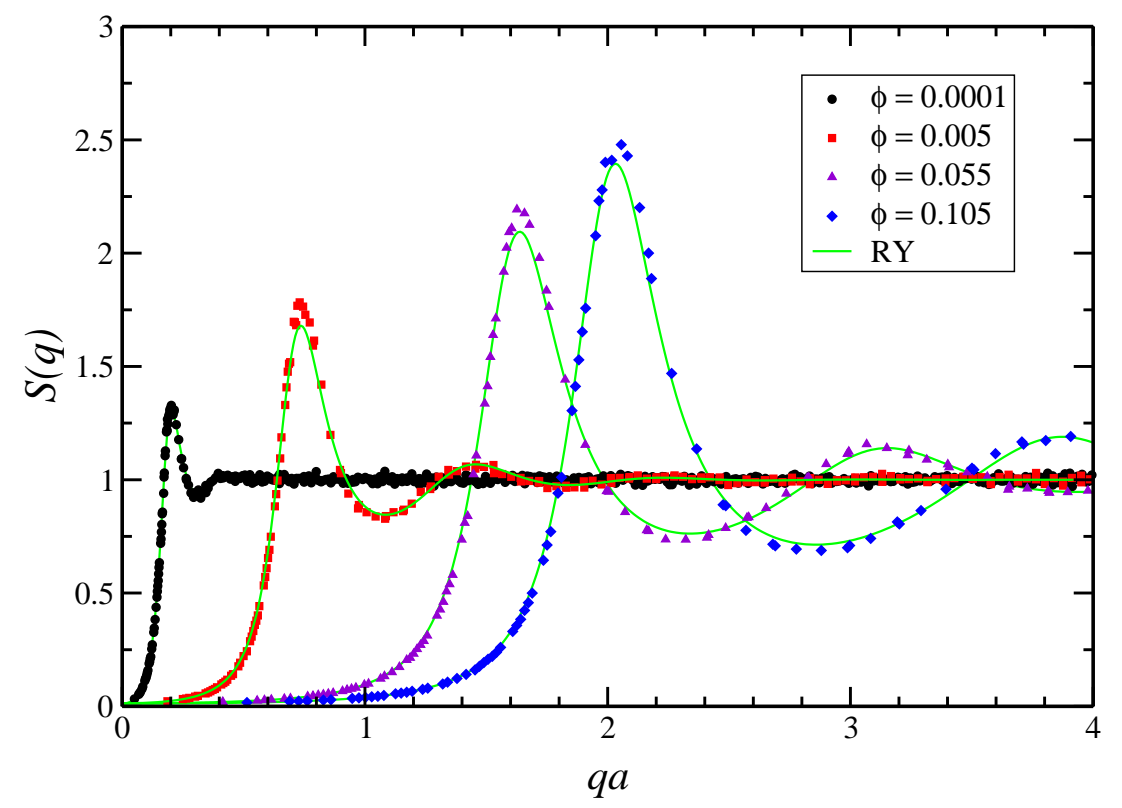

Figure 4: (Color online) Static structure factor of a de-ionized charge-stabilized suspension at volume fractions as indicated. The MC simulation data (symbols) are compared with Rogers-Young calculations (solid lines) using identical system parameters, i.e., $Z=100, L_{B}=5.62 \mathrm{~nm}$ and $n_{s}=0$.

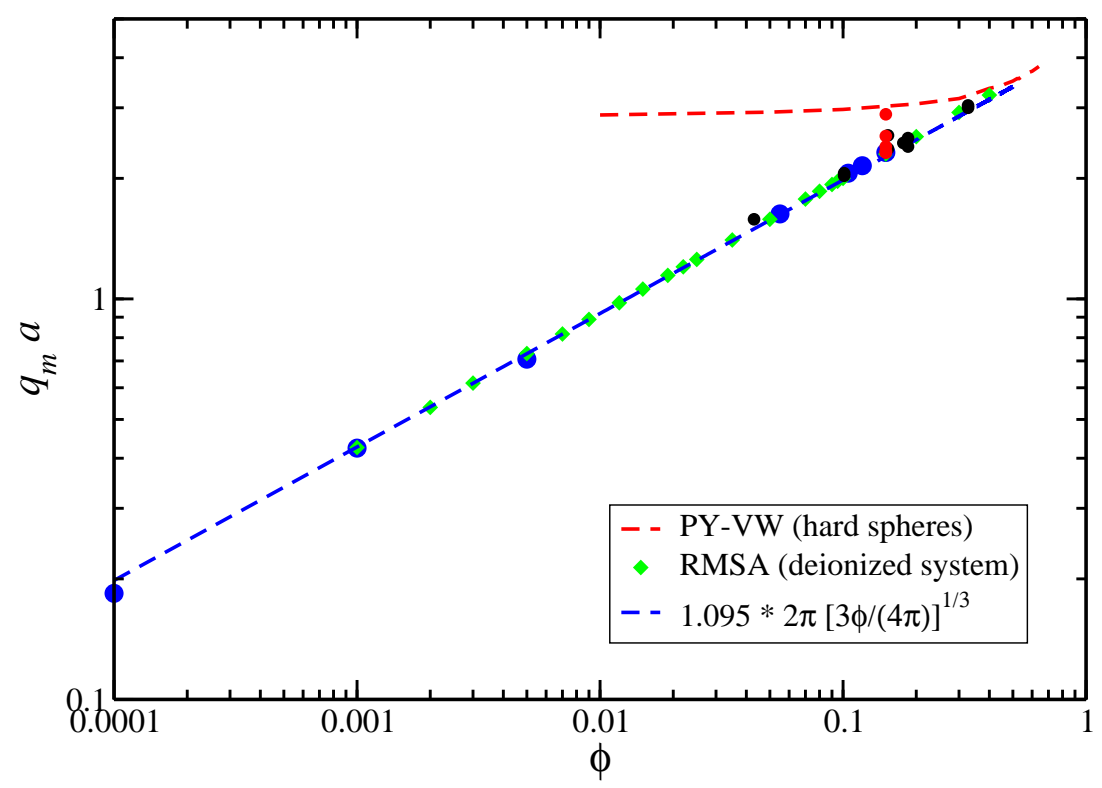

Figure 5: (Color online) Concentration dependence of the peak position, $q_{m}$, of the static structure factor of de-ionized suspension in units of the particle radius. The green diamonds are the RMSA result for $n_{s}=0$. Blue circles: MC data for $n_{s}=0$; Black circles: MC result for a collection of systems with varying amount of added salt. Red circles: MD data for a system at $\phi=0.15$ with $n_{s}$ varying from $1 \times 10^{-6}$ to $1 \times 10^{-4} \mathrm{M}$. Additionally shown is the peak-position wave number of hard spheres, as predicted by the Verlet-Weiss corrected PY scheme. 


\subsection{Short-time translational and rotational self-diffusion}

The short-time self-diffusion coefficient is a purely hydrodynamic quantity that reflects the local smalldisplacement mobility of a tracer sphere in the equilibrium dispersion. Without $\mathrm{HI}, D_{s}(\phi)$ would be equal to $D_{0}$ at any $\phi$, non-affected at all by the direct interactions. Even with $\mathrm{HI}$ included, the influence of the direct interaction forces on $D_{s}$, and on the other short-time properties, is only indirect through the effect on the equilibrium microstructure. The concentration dependence of the translational shorttime self-diffusion coefficient of neutral hard spheres is shown in Fig. 6. Our ASD simulation data, the force multipole simulation data of Ladd [21], and the experimental data of Segrè et al. [5] conform overall well with the empirical expression in Eq. (25) proposed by Lionberger and Russel. We have

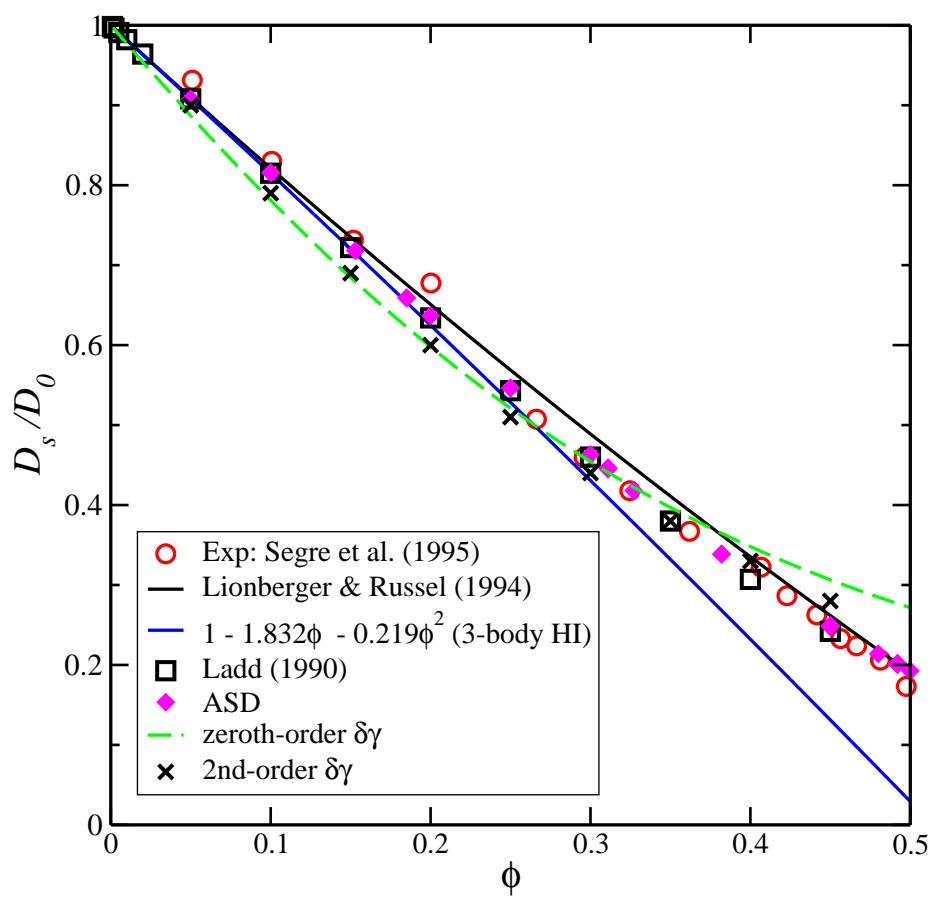

Figure 6: (Color online) Translational short-time self-diffusion coefficient of hard spheres versus volume fraction $\phi$. Comparison of the ASD and force multipole simulation data with the zeroth order and second order $\delta \gamma$ theory prediction, the 2nd order virial expansion result of Cichocki et al., the semi-empirical expression of Lionberger and Russel in Eq. (25), and the experimental data of Segrè et al. [5].

compared our ASD data with earlier ASD simulation data for $D_{s}$ by Sierou and Brady [20], and Phillips et al. [16], and we find good agreement. The 2nd-order virial expression of Cichocki et al., quoted in Eq. (20), visibly underestimates $D_{s}$ when $\phi$ is larger than 0.3 . The zeroth-order $\delta \gamma$ scheme result for $D_{s}$, which does not depend on $S(q)$, underestimates the diffusion coefficient for smaller 
values of $\phi$, whereas $D_{s}$ is overestimated for $\phi$ exceeding 0.4. Beenakker and Mazur have determined 2nd order correction terms for $D_{s}$ based on the PY input for $S(q)$ (see Fig. 7, and table III in [32]). These corrections improve the agreement of the $\delta \gamma$ scheme with the simulation data for $\phi>0.3$. In Fig. 7, we compare the concentration dependence of the $D_{s}$ for charged spheres in a de-ionized

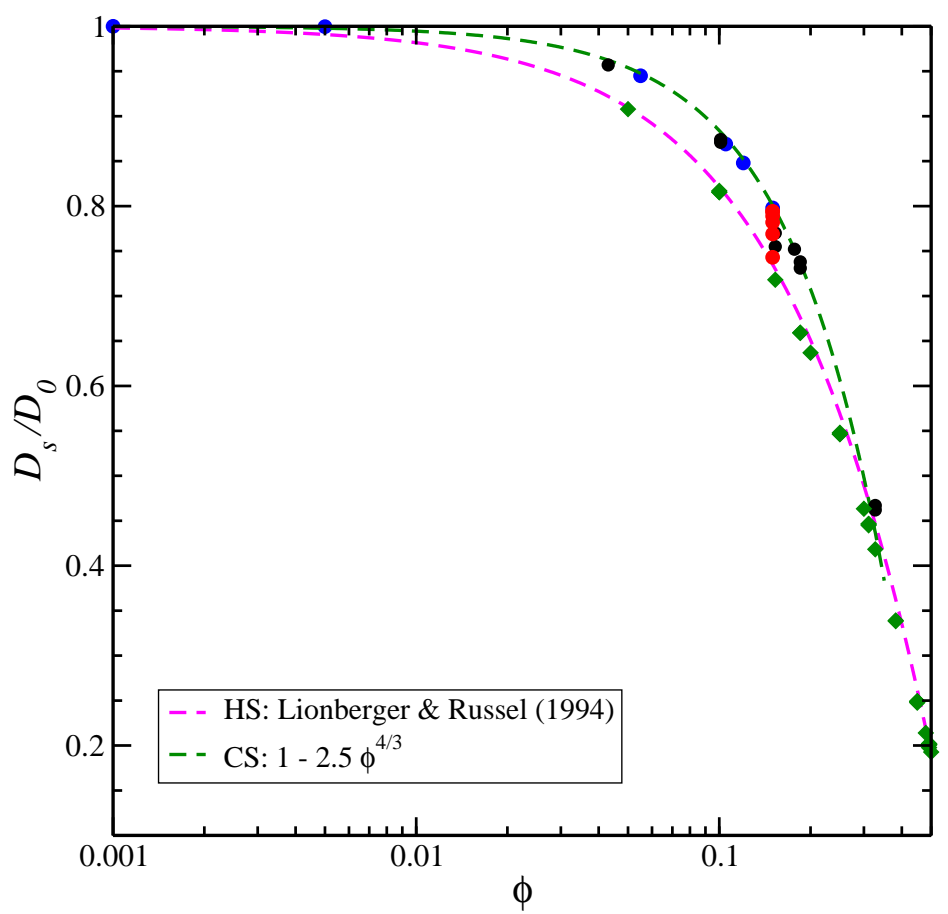

Figure 7: (Color online) Translational short-time self-diffusion coefficient of charged spheres in a salt-free suspension (labelled by CS) versus the hard-sphere result (HS). The symbols are our ASD simulation results. Green diamonds: hard spheres; blue circles: de-ionized suspension; red circles: transition from a de-ionized system, at $\phi=0.15$, to a hard-sphere-like system on increasing $n_{s}$ from $1 \times 10^{-6}$ to $1 \times 10^{-4} \mathrm{M}$; black circles: collection of systems with varying amount of added salt.

suspension with that of neutral hard spheres. The symbols denote our ASD simulation data. For all volume fractions considered, the simulation data of the de-ionized systems which are of fluidlike order conform well with the fractional concentration-scaling in Eq. (33), for a parameter value $a_{t}=2.5$. The $\phi^{4 / 3}$-dependence of $D_{s}$ has been experimentally confirmed in recent dynamic light scattering experiments on charge-stabilized suspensions treated by an ion exchange resin to remove residual salt ions [75]. The hydrodynamic self-mobility function which enters into the expression for $D_{s}$ is rather short-ranged, with a long-distance asymptotic form proportional to $r^{-4}$. Therefore, $D_{s}$ increases with decreasing ionic strength (i.e., decreasing $\kappa$ ), since the hydrodynamic slowing is weaker 
for charged spheres that repel each other over longer distances. As can be noticed from the red symbols representing a system at $\phi=0.15$ with various amounts of added salt, the addition of salt reduces $D_{s}$ towards the lower boundary set by the hard-sphere value reached in the limit of strong electrostatic screening. This generic salt-dependence of $D_{s}$ in our calculations conforms with experimental results obtained for charge-stabilized suspensions $[76,92]$. The ASD results for $D_{s}$ in all explored systems are located in between the two limiting curves for zero added salt and neutral hard spheres (see, e.g., the black circles representing a collection of very different systems with varying salt content).

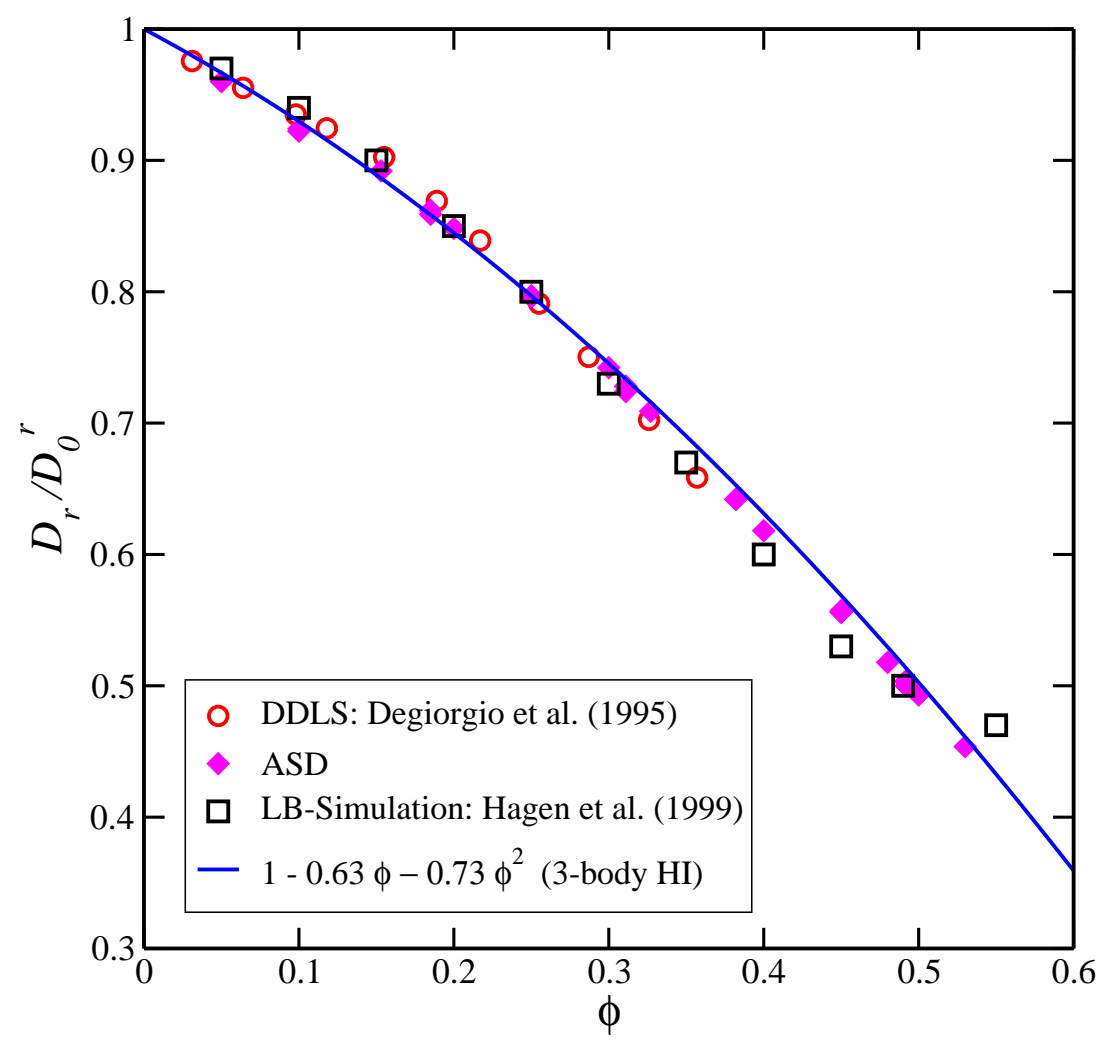

Figure 8: (Color online) Short-time rotational self-diffusion coefficient of hard spheres versus volume fraction. The symbols are our ASD results and correspond to the ones in Figs. 6 and 7. The ASD data are compared with Lattice-Boltzmann simulation data of Hagen et al. [25], the truncated virial expansion (in Eq. (21)), and the experimental data of Degiorgio et al. [74]. The 2nd-order virial expression remains valid up to $\phi=0.45$.

Our ASD simulation results for the short-time rotational self-diffusion coefficient of hard spheres are depicted in Fig. 8, and compared with earlier LB simulation data of Hagen et al., depolarized dynamic light scattering data of Degiorgio et al., and the 2nd-order virial form in Eq. (21) derived by Cichocki and coworkers [35]. We have checked our ASD data for the hard-sphere $D_{r}$ against earlier SD simulation data of Phillips et al. [16] and find good agreement. The 2nd-order virial form remains 
valid up to remarkably large volume fractions that extend to the freezing transition concentration. This suggests that all higher-order virial coefficients in $D_{s}$ are small or mutually cancel each other. In determining short-time properties such as $D_{s}, D_{r}$ and $\eta_{\infty}$, only small distance changes are probed that amount typically to a small fraction of the particle diameter. Therefore, short-time quantities are rather insensitive to qualitative changes in the microstructure, and cross over smoothly into the liquid-solid coexistence regime. This should be contrasted to long-time dynamic properties, which may change drastically in their behavior at equilibrium and non-equilibrium transition points.

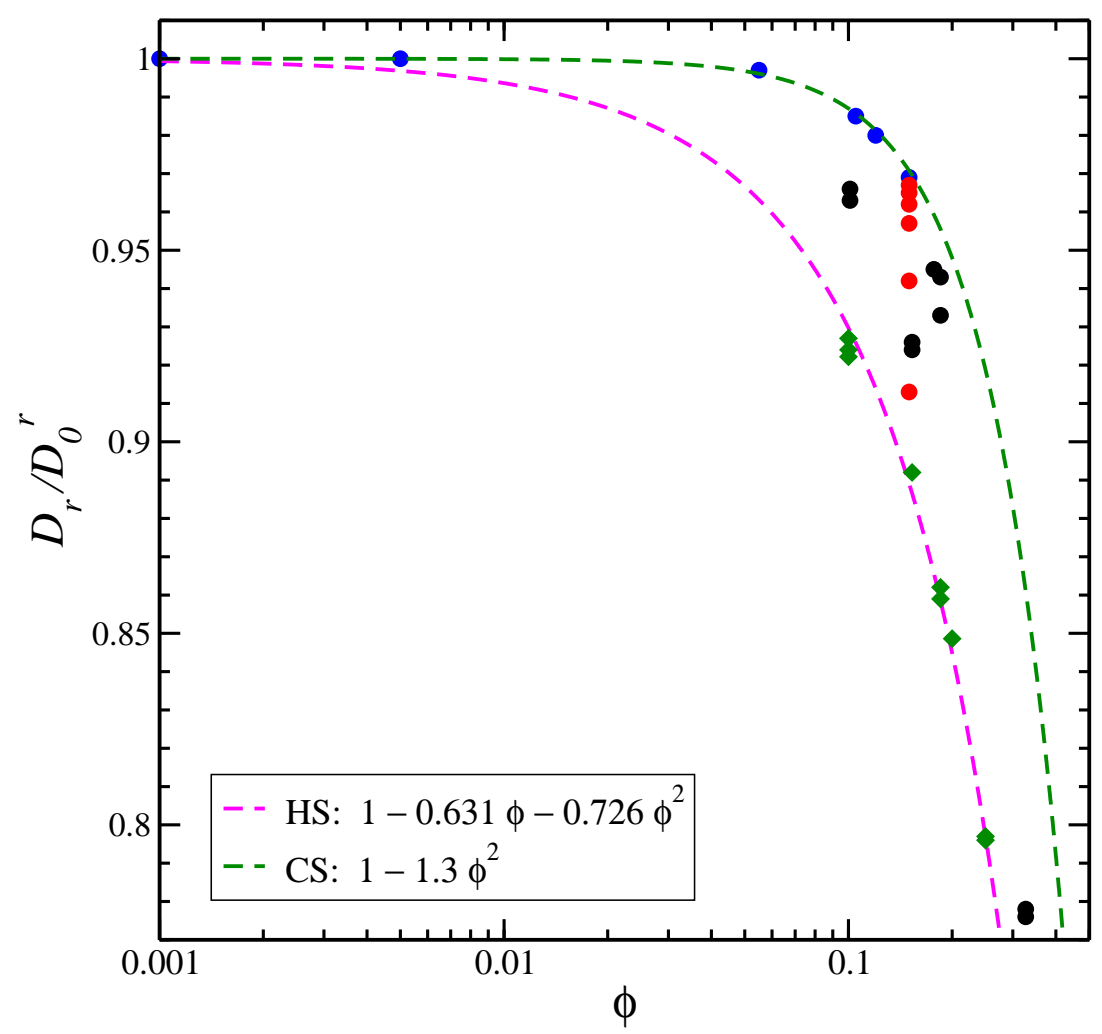

Figure 9: (Color online) Short-time rotational self-diffusion coefficient of a de-ionized suspension of charged spheres (CS), in comparison to the $D_{r}$ of neutral spheres (HS). The symbols are our ASD simulation results, with same symbols and color coding as in Fig. 7. The quadratic scaling form in Eq. (34), which accounts for far-field 3-body HI corrections, remains valid up to remarkably large $\phi$. The $D_{r}$ for systems with added salt is bounded from above and below, respectively, by the two limiting curves describing de-ionized charged-sphere and neutral hard-sphere systems.

Our ASD simulation data (symbols) for the short-time rotational diffusion coefficient of chargestabilized and neutral colloidal spheres are shown in Fig. 9. The quadratic scaling form in Eq. (34), valid for deionized systems and accounting for far-field 3-body HI, is seen to apply up to remarkably 
large values of $\phi$. It should be stressed here that Eq. (34) is not the result of a standard virial expansion, since for zero added salt the system is dilute only regarding the hydrodynamic interactions, which can be described thus on the two-body and leading-order three-body level, but non-dilute regarding direct interactions. The radial distribution function in these systems has pronounced oscillations typical for strong fluid-like ordering. The scaling prediction in Eq. (34) has been confirmed additionally by LB simulations of Hagen et al. [25], which show that it applies accurately up to $\phi \approx 0.3$. The coefficient $D_{r}$ of charge-stabilized suspensions decreases with increasing amount of added salt ions, for the same reason as discussed earlier in the context of $D_{s}$. For short-time rotational diffusion, the hydrodynamic self-mobility tensor associated with $D_{r}$ decays asymptotically as $r^{-6}$, i.e., by two powers in $r$ stronger than the hydrodynamic mobility related to translational self-diffusion. This is the reason why $D_{r}$ is quite sensitive to the ionic strength, so that a small residual amount of excess ions leads to a curve for $D_{r}$ located below the upper limiting curve described by Eq. (34). The pronounced sensitivity of $D_{r}$ on the ionic strength has been observed also experimentally [40]. For larger amounts of added salt, the lower limiting curve for $D_{r}$ describing neutral hard spheres is reached (see red symbols in Fig. 9).

\subsection{High-frequency limiting viscosity}

We discuss next the high-frequency limiting suspension viscosity measured by high-frequency and low-amplitude shear oscillation rheometers in the Newtonian regime where shear-thinning is absent. Under these conditions, the equilibrium microstructure remains unaffected by the imposed shear flow. Computer simulation and theoretical results for the $\eta_{\infty}$ of colloidal hard spheres are displayed in Fig. 10. The expression in Eq. (27) given by Ladd, which fits his simulation data obtained up to $\phi=0.45$ (see table IV in [21]), is seen to apply to even larger values of $\phi$ where it conforms also with our ASD simulation data, and the ones of Sierou and Brady [20]. There is a wealth of experimental data available for $\eta_{\infty}$ with show a significant stray due to statistical errors and size polydispersity. Instead of including experimentala data, we refer to the Lionberger-Russel formula in Eq. (26) as an representative for the average of these data. The empirical Lionberger-Russel form for $\eta_{\infty}$ agrees overall well with the simulation data. The 3rd-order virial expansion result in Eq. (23) is applicable up to $\phi>0.3$. At larger $\phi$, the uprise in $\eta_{\infty}$ is underestimated. The 2nd-order $\delta \gamma$ theory result for $\eta_{\infty}$, which has been calculated by Beenakker using the PY-S(q) as input, agrees overall well with the ASD simulation data in the full concentration range up to $\phi \approx 0.45$ where it is applicable. 
This is remarkable since the $\delta \gamma$ theory accounts only approximately for near-field HI and disregards lubrication. Moreover, in Fig. 10 we show the result for $\eta_{\infty}$ described by the modified Saito expression

$$
\frac{\eta_{\infty}}{\eta_{0}}=1+\frac{5}{2} \phi \frac{1+1.0009 \phi+0.63 \Phi^{2}}{1-\phi-1.0009 \phi^{2}-0.63 \Phi^{3}}
$$

which has been derived by Cichocki et al. using their third-order virial expression result for the shorttime viscosity [37]. This expression strongly overestimates $\eta_{\infty}$ when $\phi$ exceeds 0.4 . Out of the present comparison of analytic viscosity expressions, Eq. (27) emerges as a handy formula which describes the overall $\phi$-dependence of $\eta_{\infty}$ very well. Our ASD simulation data for the $\eta_{\infty}$ of a de-ionized

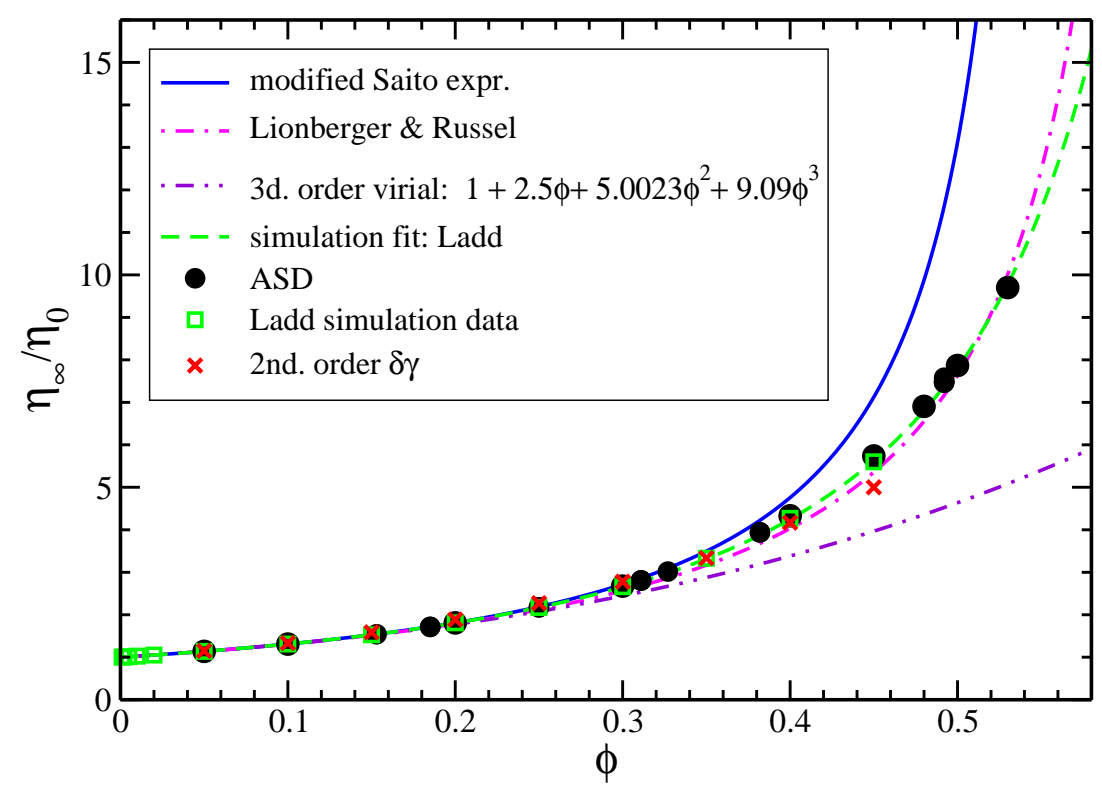

Figure 10: (Color online) High-frequency limiting viscosity, $\eta_{\infty}$, of colloidal hard spheres versus $\phi$. Displayed are our ASD simulation data in comparison with the force multipole simulation data of Ladd [21], the third-order truncated virial expansion result in Eq. (23), the semi-empirical Lionberger-Russel expression in Eq. (26), the simulation fitting formula of Ladd in Eq. (27), the modified Saito expression of Cichocki et al. in Eq. (45), and the 2 nd order $\delta \gamma$-PY result taken from [43].

charge-stabilized suspension are shown in Fig. 11, and compared with the analytic expression in Eq. (44) based on a schematic model calculation. This expression conforms with the simulation data overall quite well for the liquid-state volume fractions considered (note that $S\left(q_{m}\right) \approx 2.8$ at $\phi=0.15$ ). At smaller $\phi<0.1$, the first-order Einstein term part in Eq. (44) dominates. Furthermore, we display the result for $\eta_{\infty}$ described in Eq. (40) which fully accounts of 2-body HI and is based on the RMSA input for $g(r)$. This result fully conforms with Eq. (44) in the whole $\phi$-range considered. 
The modest increase of $\eta_{\infty}$ with increasing volume fraction, and its very weak dependence on the ionic strength noticed from ASD simulations for varying $n_{s}$ (not shown here) are features consistent with the experimental findings of Bergenholtz et al. [76]. In fact, as can be noticed in the inset of Fig. 11 where ASD viscosity data for hard spheres are compared with those of two deionized system, the differences in the viscosities are quite small. The differences are largest at intermediate volume fractions where two-body HI prevail. They become smaller at larger $\phi$ where the particles are close to each other and near-field many-body HI is strong. The curves for $\eta_{\infty}(\phi)$ in systems with added salt are

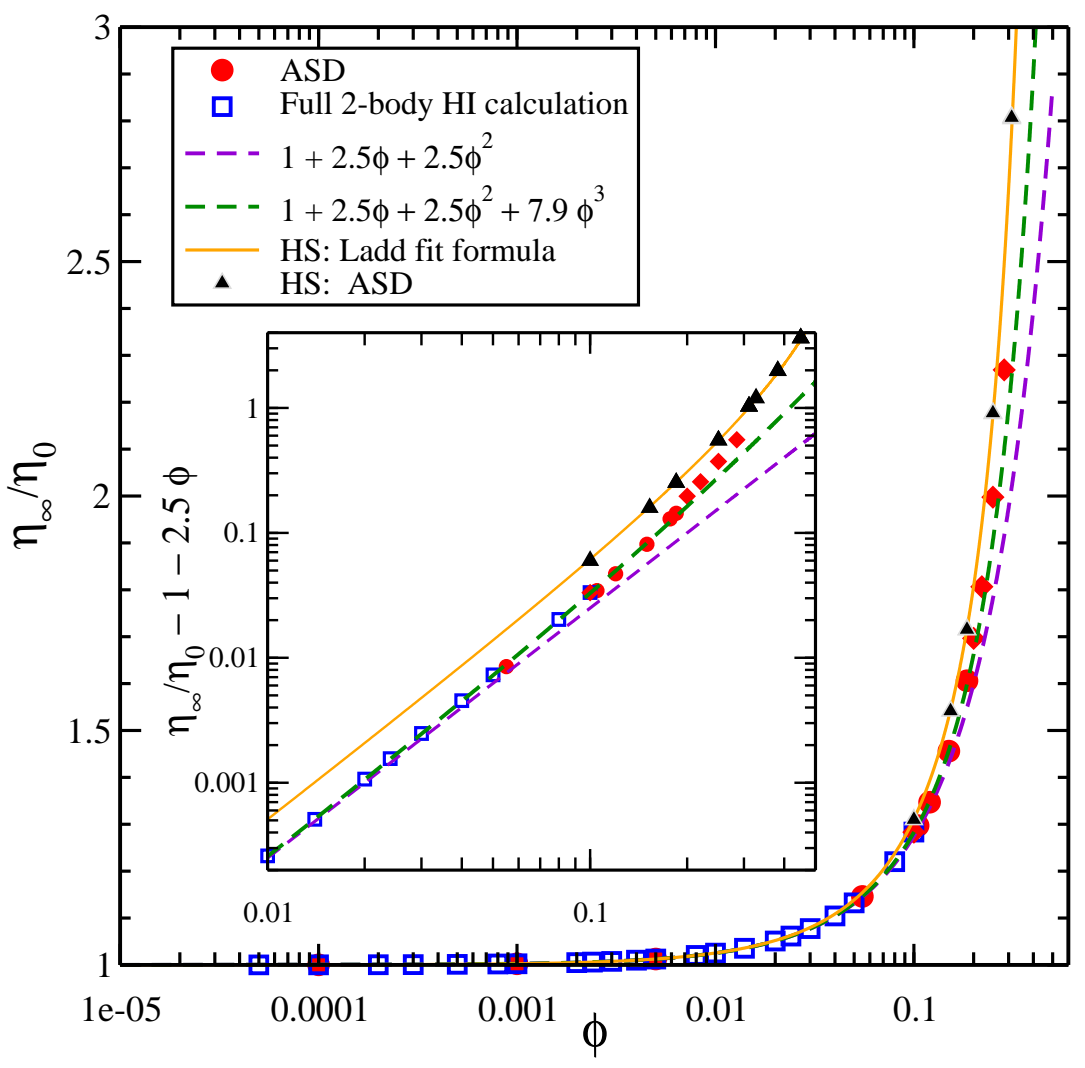

Figure 11: (Color online) High-frequency limiting viscosity of two de-ionized suspensions of charged spheres (Circles: $Z=100, a=100 \mathrm{~nm}, L_{B}=5.62 \mathrm{~nm}, n_{s}=0$; diamonds: $Z=70, a=25 \mathrm{~nm}, L_{B}=0.71 \mathrm{~nm}$, $n_{s}=0$ ). The ASD simulation data are overall well described by Eq. (44) which derives from a schematic model for $g(r)$ using the leading-order far-field HI contribution. For an expanded view of the differences, the inset shows the excess short-time viscosity.

all located in between the two limiting curves for zero and infinite amount of added salt. Consistent with a corresponding behavior of $D_{s}(\phi)$, the $\eta_{\infty}$ of a dilute charge-stabilized suspension is smaller than that of a hard-sphere system at the same $\phi$ (compare Eq. (23) with Eq. (44)), reflecting the weaker 
hydrodynamic dissipation of in charged-sphere systems due to the depletion of neighboring spheres near contact caused by electrostatic repulsion. The experimental data in [76] and [92] conform with the theoretically predicted trends even at large values of $\phi$. At very large volume fractions, however, many-body near-field HI come into play even in low-salt systems. Then, a hard-sphere-like behavior of $\eta_{\infty}$ is approached, and Eqs. (40) and (44) do not apply any more (see the inset in Fig. 11).

\subsection{Short-time sedimentation coefficient}

We notice from comparing Eq. (22) with (35), that there is a remarkable difference at lower $\phi$ in the concentration dependence of the short-time sedimentation velocity, $U_{s}$, between hard spheres and deionized charged-sphere systems. Results for the sedimentation coefficient of hard spheres obtained by various methods are included in Fig. 12. As discussed earlier, near-field HI only have a small influence on the sedimentation coefficient. This is the reason why the long-time sedimentation coefficient is only slightly smaller than the short-time one, and why the $\delta \gamma$ theory result, with its approximate account of near-field HI without lubrication correction, agrees decently well with the force multipoles simulation result of Ladd [21], and the LB simulation result of Segrè et al. [5]. The LB data shown in the figure have been obtained from multiplying the LB data for $D_{C} / D_{0}$ in [5] with $S_{\mathrm{CS}}(0)$. The RotnePrager approximation for $U_{s}$ given in Eq. (39) overestimates the simulated sedimentation velocity, with growing difference for increasing concentration. Yet, the Rotne-Prager $U_{s}$ compares reasonably well overall with the simulation data for small to intermediate values of $\phi$, which reflects the weak near-field $\mathrm{HI}$ dependence of $U_{s}$ for volume fractions which are not very large. The 2nd-order virial result in Eq. (22) for $U_{s}$ is valid for $\phi \leq 0.1$ only, as signalled by the sign change in going from the first to the second virial coefficient. Whereas the sedimentation velocity is little affected by memory effects for reasons discussed already before, the zero-shear static viscosity, $\eta(\phi)$, and the long-time self-diffusion coefficient, $D_{l}(\phi)$, differ substantially from their short-time counterparts. At long times, direct forces influence the transport coefficients directly through a perturbation of the equilibrium microstructure caused by the motion of a tagged sphere in the case of self-diffusion, and the shear flow distortion in the case of the viscosity. To illustrate the pronounced difference between short-and long-time viscosities, in Fig. 13 we have also included the result for $\eta_{0} / \eta_{\infty}$ according to Eq. (27), and the inverse of the static viscosity $\eta_{0} / \eta$ as predicted by the hydrodynamically rescaled mode-coupling scheme (MCS). The latter compares well with experimental viscosity data on hard spheres [52]. For a 


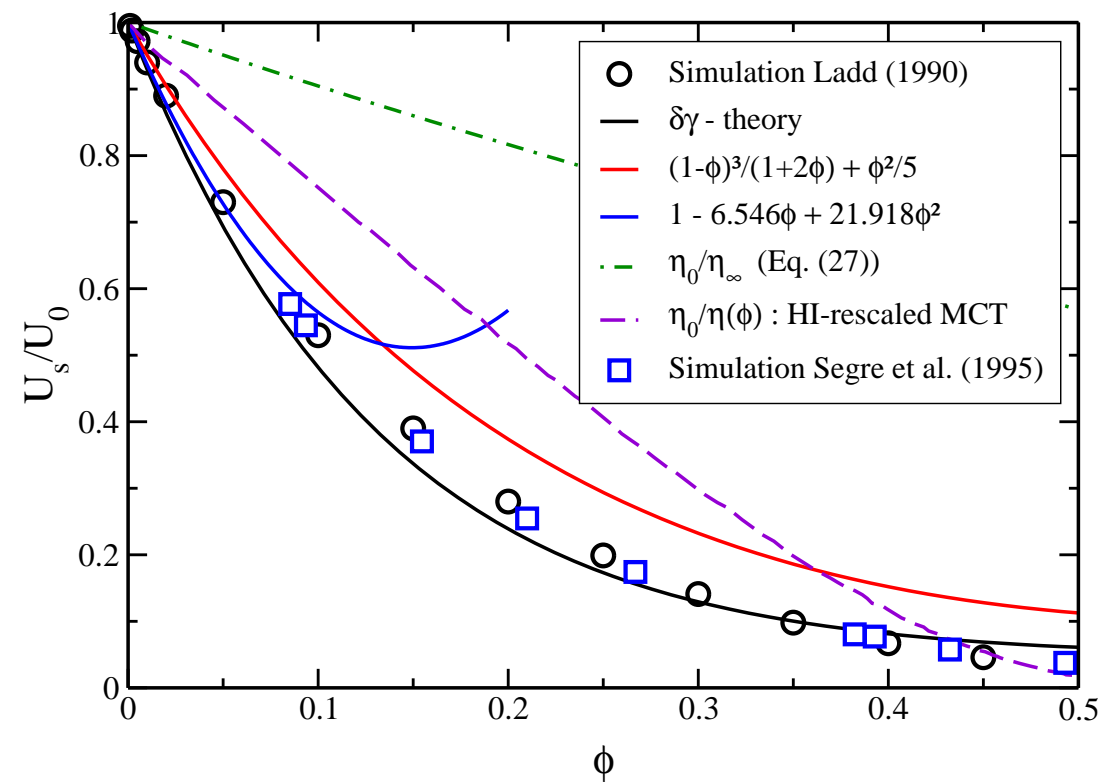

Figure 12: (Color online) Short-time sedimentation coefficient, $U_{s} / U_{0}$, of a homogeneous hard-sphere suspension. The 2nd-order virial and Rotne-Prager approximation results, and the zeroth-order $\delta \gamma$ scheme prediction are compared with the accurate computer simulation results of Ladd [21], and LB simulations of Segrè et al. [5]. Note here the strong difference between $\eta_{0} / \eta_{\infty}(\phi)$ and $\eta_{0} / \eta(\phi)$. 
discussion of the static viscosity of colloidal hard spheres see also $[18,93]$. In linear response theory, the self-diffusion coefficient is obtained by considering a weak external force applied to a single tagged sphere. For hard spheres, the influence of surrounding neutrally buoyant spheres can be then described approximately by the high-frequency (in case of $D_{s}$ ) or the static viscosity (in case of $D_{l}$ ), giving rise to the approximate validity of a generalized Stokes-Einstein relation between the self-diffusion coefficient and the viscosity (see subsection 3.7 for a discussion of this relation). It is apparent from Fig. 13 that such a simple mean-field-type picture is not valid in the case of sedimentation, since it would imply that $U_{s} / U_{0} \approx \eta_{0} / \eta_{\infty}$.

Recent experimental data for the sedimentation coefficient of a de-ionized charge-stabilized suspension, obtained from a small- $q$ scattering experiment [50], are shown in Fig. 13. These data are in good agreement with Eq. (35) for $a_{s}=1.8$, whose validity is a consequence of the dominating far-field 2-body HI, and the scaling relation $\bar{r} \sim \phi^{-1 / 3}$ obeyed in low-salinity systems for $\phi \leq 0.1$ [87]. That charged spheres sediment more slowly than neutral ones can be rationalized as follows: For charged spheres at low salinity, near-contact configurations are very unlikely due to the strong electric repulsion. On the average, this causes an enlarged laminar friction between the backflowing solvent part which has its origin in the non-zero total force on the spheres, and the solvent layers adjacent to the sedimenting spheres which are dragged along. Hasimoto [94] and Saffman [95] have shown that a simple cubic lattice of widely separated spheres sediment with a velocity in accord with Eq. (13), but for a slightly smaller parameter of $a_{s}=1.76$. For the scaling relation in Eq. (35) to be valid, however, a long-range periodicity of the particle configuration is not necessary. What is required only is a strong and long-range inter-particle repulsion, which creates around each sphere a well-developed shell of next neighbors of radius scaling as $n^{-1 / 3}$.

\subsection{Collective diffusion coefficient}

On dividing $U_{s} / U_{0}$ by $S(0)$, the short-time collective diffusion coefficient, $D_{c} / D_{0}$, is obtained. This coefficient can be measured in a low- $q$ dynamic scattering experiment or, alternatively, in a macroscopic gradient diffusion experiment on ignoring in the latter case the small difference between longtime and short-time collective diffusion. The simulation results of Ladd [21] and Segre et al. [5] for hard spheres show a weak concentration dependence of $D_{c}$ (see Fig. 14), which reflects very similar $\phi$-dependencies of $U_{s}$ and $S(0)$. Up to $\phi=0.4$, the simulation data follow closely the 2nd-order virial 


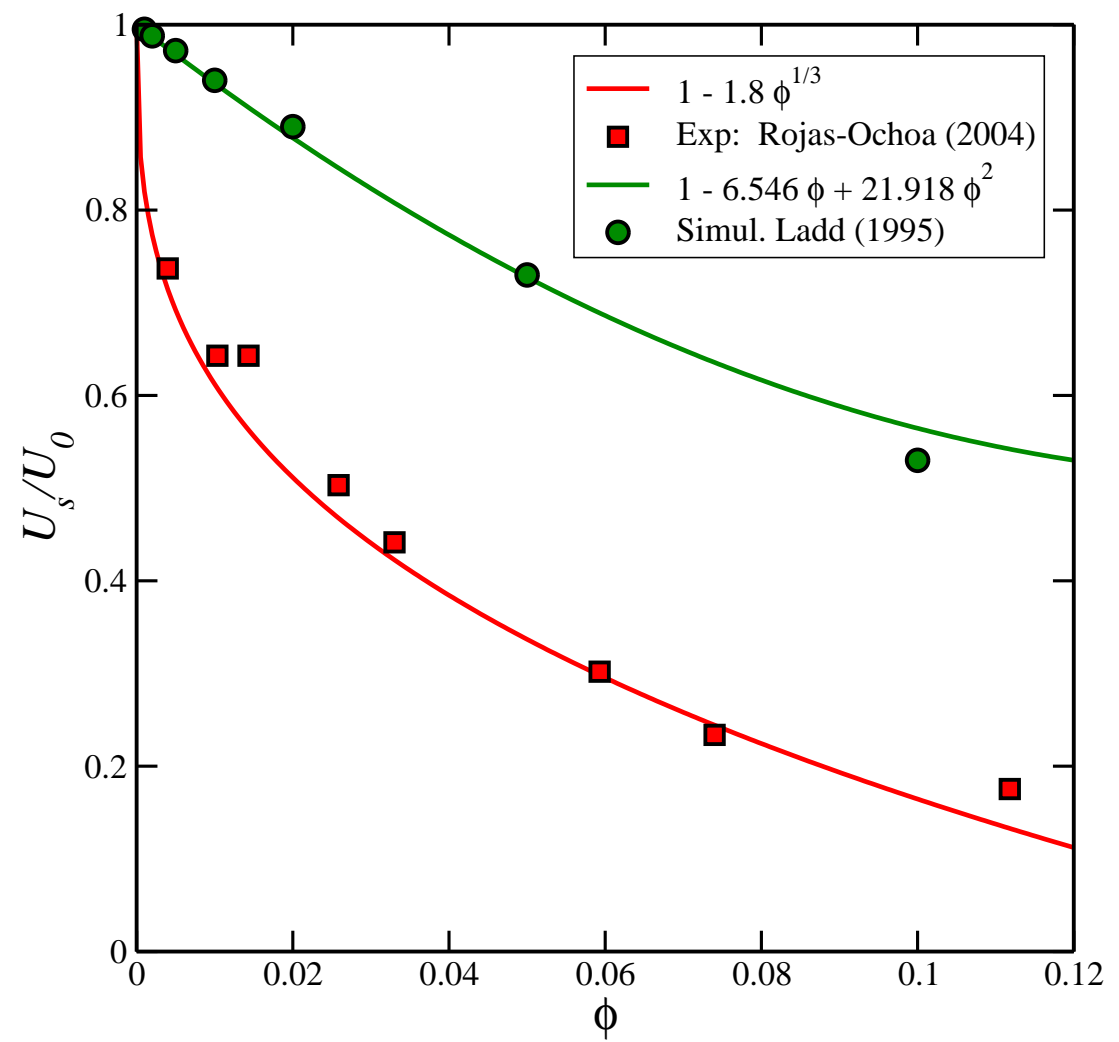

Figure 13: (Color online) Sedimentation coefficient, $U_{s} / U_{0}$, of a de-ionized charge-stabilized suspension versus $\phi$. Experimental data are taken from [50], and compared with the scaling form in Eq. (35) using $a_{s}=1.8$. The sedimentation coefficient of hard spheres as described by Eq. (22) is shown for comparison. 
result in Eq. (24) for $D_{c}$ which, unlike to $U_{s}$, points to a strong mutual cancellation of higher-order virial contributions in the case of $D_{c}$. The $\delta \gamma$ result for $D_{c}$ shown in Fig. 14 has been obtained from dividing the $\delta \gamma-U_{s} / U_{0}$ depicted in Fig. 12 through $S_{\mathrm{CS}}(0)$. The underestimation of $D_{c}$ at small $\phi$,

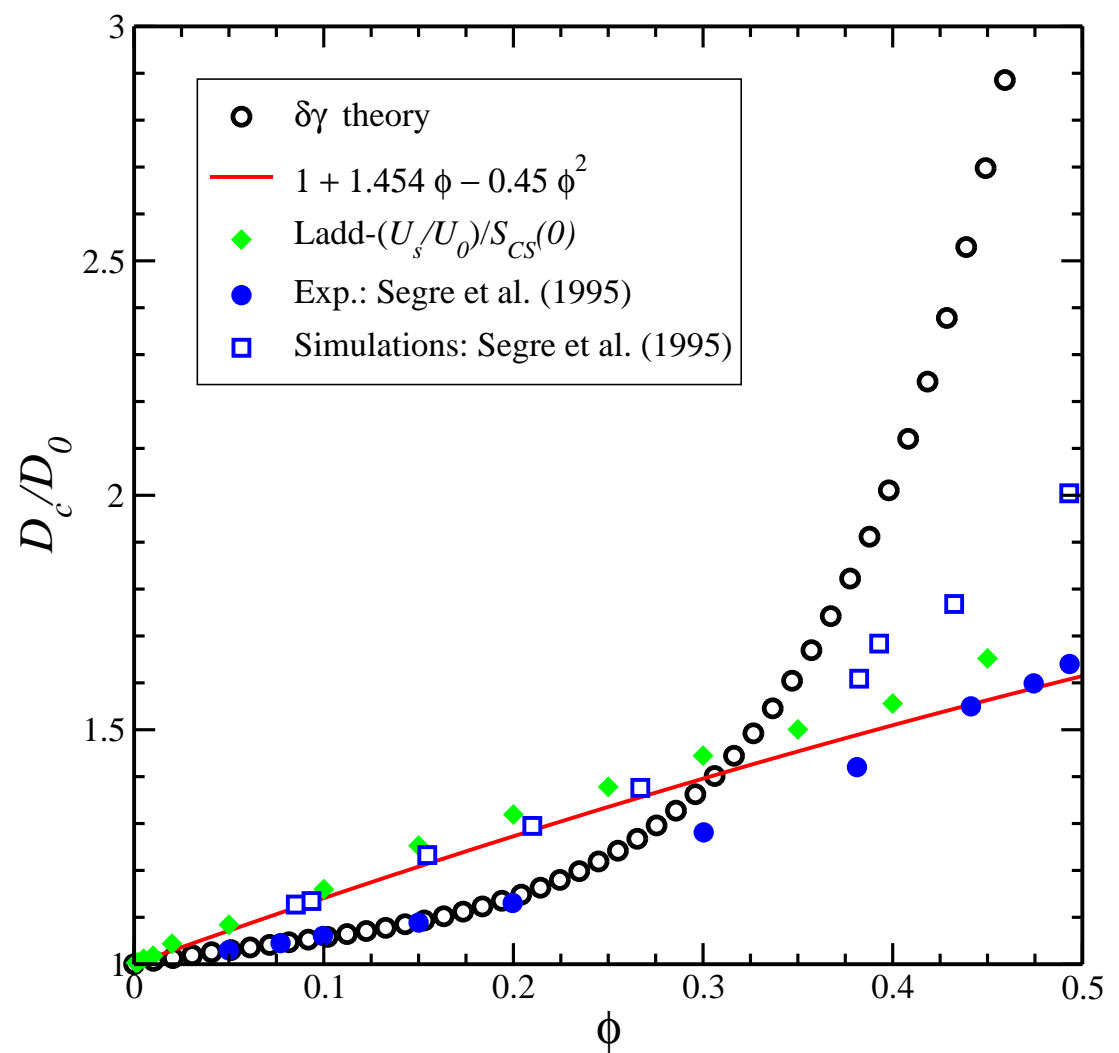

Figure 14: (Color online) Short-time collective diffusion coefficient, $D_{c}=D(q \rightarrow 0)$, of hard spheres. Comparison between the simulation data of Ladd [21], obtained from dividing the simulated $U_{s} / U_{0}$ by $S_{\mathrm{CS}}(0)$, LB simulation data and dynamic light scattering data of Segrè et al. [5], zeroth-order $\delta \gamma$ theory prediction, and the 2nd-order virial result in Eq. (24).

and its gross overestimation for $\phi>0.4$, reflects a corresponding behavior in the $\delta \gamma$ result for $U_{s}$, but is here more visible due to the division by $S_{\mathrm{CS}}(0)$. We note here that the $\delta \gamma$ result for $U_{s}$ remains practically unchanged when, in place of the PY $S(q)$, the Verlet-Weiss corrected structure factor is used [44]. Significant deviations between experimental and simulation data are visible even at smaller concentrations where the 2nd-order virial expansion applies. As noted in [5], these deviations might result from the lack of experimental data at low enough wavenumbers to provide reliable extrapolations to $q=0$.

In charge-stabilized suspensions, $D_{c}$ increases more rapidly with concentration and typically passes 
through a distinct maximum which increases (decreases) and shifts to larger (smaller) values of $\phi$ for growing particle charge (salt content). The observed maximum arises since, at larger $\phi$, the hydrodynamic hindrance determined by $U_{s}$ overcompensates the small osmotic compressibility proportional to $S(0)$. For recent $\delta \gamma$ theory calculations of the $D_{c}$ for charge-stabilized systems describing experimental results for globular charged proteins, we refer to [6].

\subsection{Hydrodynamic and short-time diffusion functions}

Our ASD simulation results for the wave-number dependent hydrodynamic function $H(q)$ have been obtained using the finite-size correction scheme in Eq. (17), initially used for hard-sphere suspensions $[21,22]$. We have verified that this scheme gives a unique master curve also for charged spheres, over the whole explored range of system sizes $N$, volume fractions, salt contents and effective charges. Fig. 15 shows the result of the finite-size correction for a low-salt suspension using an ascending number $N=125-860$ of spheres in the basis simulation box. A unique hydrodynamic function $H(q)$ is obtained that is practically independent of $N$.

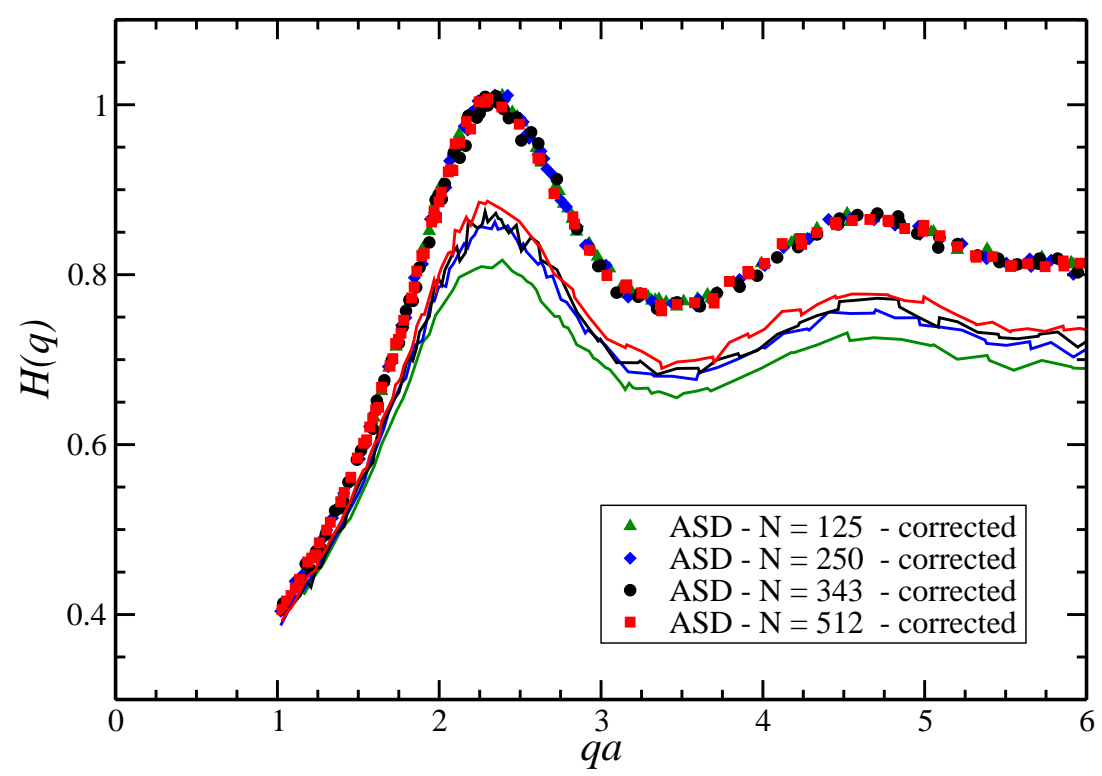

Figure 15: (Color online) Uncorrected ASD-simulated hydrodynamic function, $H_{N}(q)$, of a charge-stabilized suspension (lines) with $\phi=0.123, Z=1400, a=82.5 \mathrm{~nm}$, and $L_{B}=0.71 \mathrm{~nm}$, for various numbers $N$ of simulated spheres as indicated. The finite-size corrected functions (symbols), obtained using Eq. (17), collapse on a single curve that is identified as $H(q)$.

In Fig. 16 we compare the zeroth-order $\delta \gamma$ scheme results for the $H(q)$ of hard spheres, calculated 


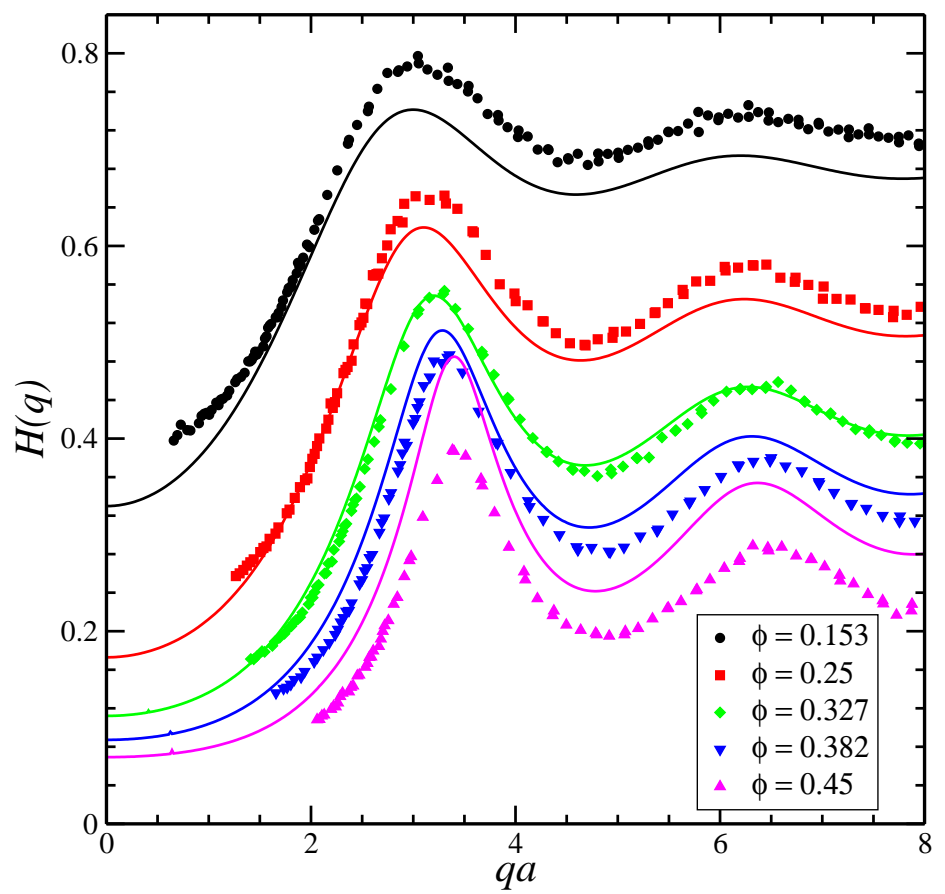

Figure 16: (Color online) ASD simulation results for the hydrodynamic function of hard spheres in comparison with the $\delta \gamma$ theory predictions. 
using Eqs. 18 and 19 and the PY input for $S(q)$, with our ASD simulation data. The simulated $H(q)$ are overall underestimated by the $\delta \gamma$ scheme for volume concentrations $\phi<0.3$, and overestimated for $\phi>0.4$. This reflects a similar behavior of the sedimentation coefficient depicted in Fig. 12. The ASD simulation results for the corresponding short-time diffusion function $D(q)$ are shown in Fig. 17. For $q \ll q_{m}$, the diffusion function is larger than the free diffusion coefficient due to the speed-up of collective diffusion by the low osmotic compressibility. The diffusion function attains its minimal value smaller than $D_{0}$ at the position of the principal peak in $S(q)$, corresponding to the slowest decay of density fluctuation of wavelength $2 \pi / q_{m}$. With increasing $\phi$ the next-neighbor cage stiffens and $D\left(q_{m}\right)$ gets smaller. For $q \gg q_{m}$, only small distances are probed corresponding to single-particle motion: Here, $D(q)$ becomes equal to the short-time self-diffusion coefficient. The principal peak

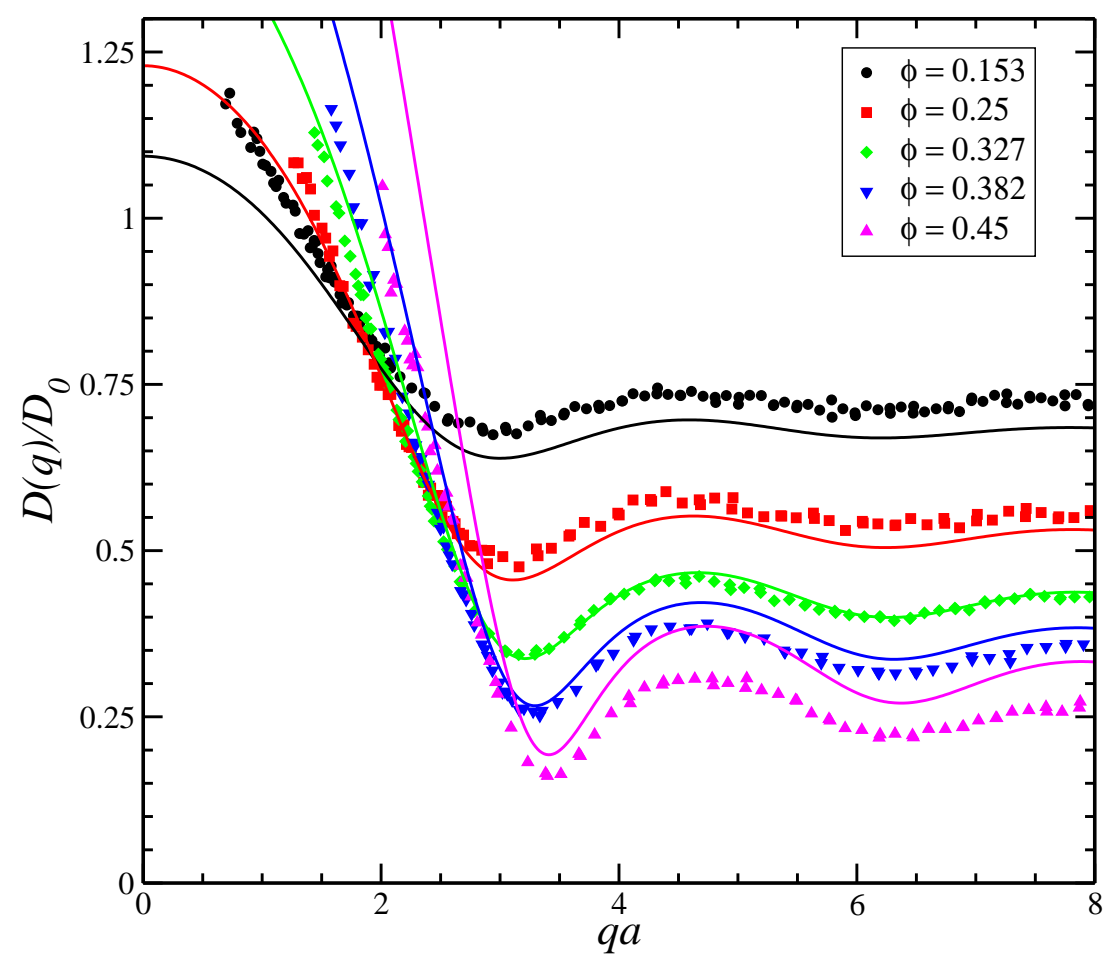

Figure 17: (Color online) ASD simulation predictions for the short-time diffusion function of hard spheres in comparison with the $\delta \gamma$ theory predictions.

height, $H\left(q_{m}\right)$, of hard spheres as a function of the concentration is displayed in Fig. 18. The ASD simulation data, which cover a the full liquid-state concentration range up to $\phi=0.5$, as well as the LB simulation and experimental data of Segrè et al. [5], are well represented by the linear form in Eq. (28). This expression for $H\left(q_{m}\right)$ conforms also to the exact numerical value in the dilute limit, which 
we have determined using the exact numerical form of the 2-body hydrodynamic mobilities. Segrè et al. [5] give a polynomial fitting formula, $D_{0} / D\left(q_{m}\right)_{\mathrm{LB}}=1-2 \phi+58 \phi^{2}-220 \phi^{3}+347 \phi^{4}$ that describes their LB data for $D\left(q_{m}\right)$ within $4 \%$ of accuracy for volume fractions $\phi>0.1$. Combining this with Eq. (30), a LB fitting formula for $H\left(q_{m}\right)$ is obtained in overall good agreement with Eq. (28) provided that $\phi>0.13$. The linear concentration-scaling of $H\left(q_{m}\right)$, valid in the whole fluid regime, is an empirical finding which we can not explain so far in terms of a simple physical picture. Like in sedimentation and collective diffusion, the $\delta \gamma$-theory underestimates $H\left(q_{m}\right)$ at smaller $\phi$, and overestimates it for $\phi>0.4$. The analytic result for the cage diffusion coefficient, $D\left(q_{m}\right)$, defined by the ratio of Eqs.

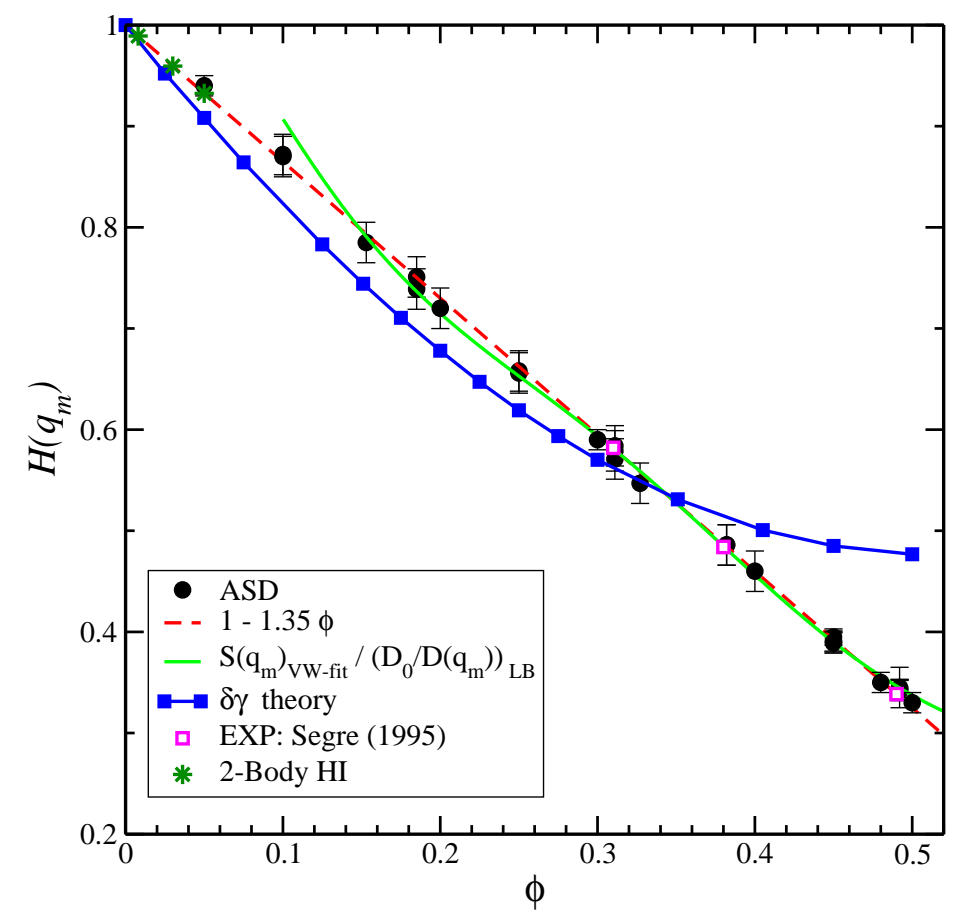

Figure 18: (Color online) Peak height, $H\left(q_{m}\right)$, of the hydrodynamic function of hard spheres. ASD simulation data are compared with the LB predictions of Behrend et al. [5], the $\delta \gamma$ theory with PY input, experimental data of Segrè et al [5], and the empirical expression $1-1.35 \phi$ in Eq. (28).

(28) and (30), is seen in Fig. 19 to be in excellent agreement with the experimental data of Segrè et al. [5], and our simulation data. This reflects again the accuracy of Eq. (28) in describing the peak height of the hydrodynamic function of hard spheres.

ASD simulation results for the $H(q)$ of a charge-stabilized suspension in dependence on the volume fraction, and the amount of added electrolyte, are included in Figs. 20 and 21, respectively.

According to Fig. 20, the $q$-dependence of $H(q)$ is well captured by the $\delta \gamma$ scheme, although 


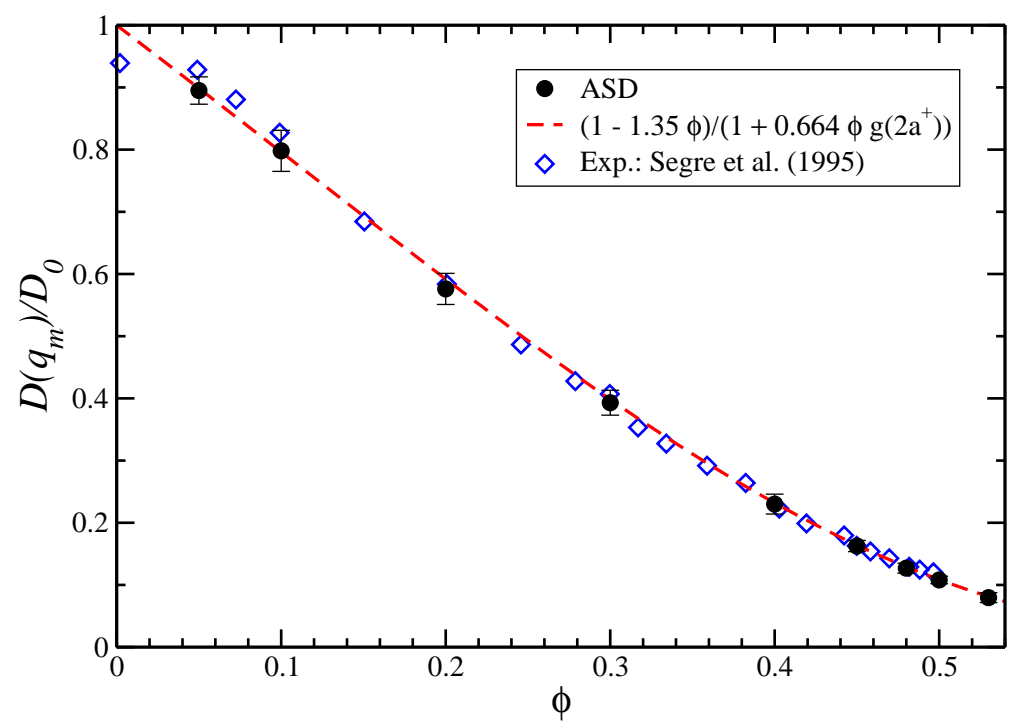

Figure 19: (Color online) Cage diffusion coefficient, $D\left(q_{m}\right)$, of colloidal hard spheres versus volume fraction. The analytic expression for $D\left(q_{m}\right)$, defined by the ratio of Eqs. (28) and (30), is tested against ASD simulation data, and the experimental data of Segrè et al. [5].

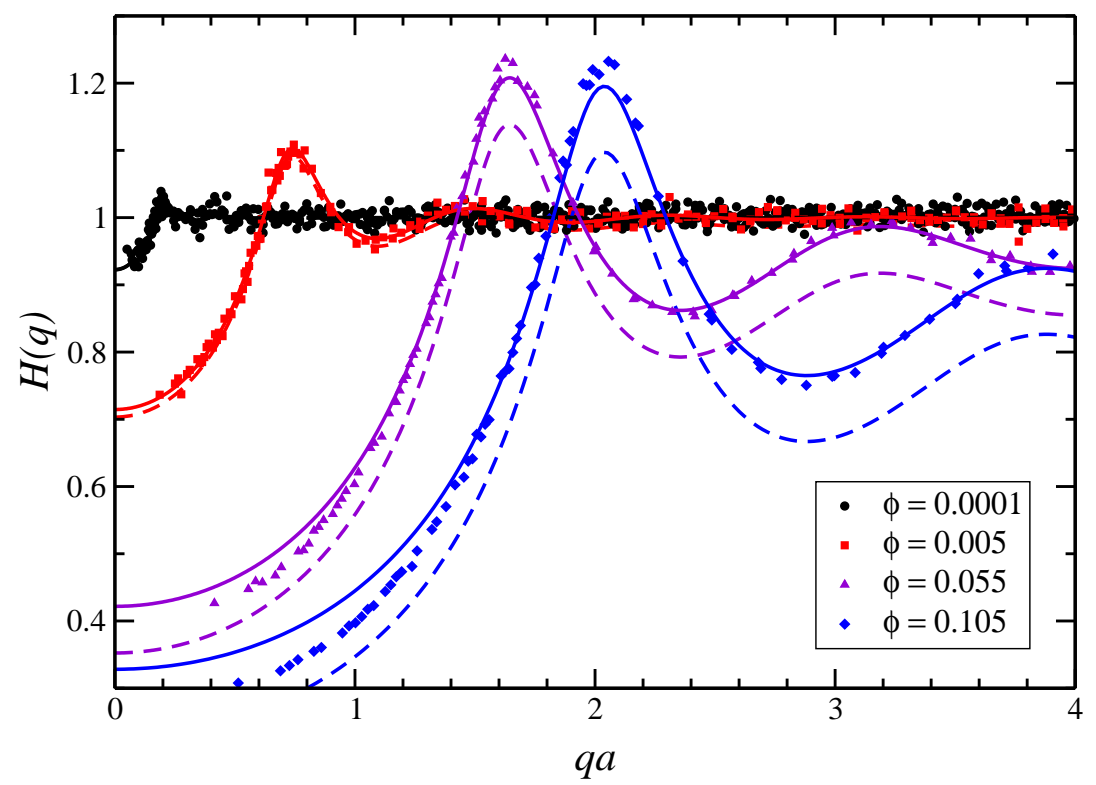

Figure 20: (Color online) ASD-simulated hydrodynamic function $H(q)$ of charged spheres at zero added salt content, in dependence on $\phi$. The $\delta \gamma$ theory result (dashed lines) and the self-part corrected $\delta \gamma$ theory result (solid lines) are included for comparison. 
its predictions are consistently lower than the simulation data. The differences between ASD and $\delta \gamma$ theory become larger with increasing $\phi$ and decreasing salt content. The underestimation of the true $H(q)$ by the zeroth-order $\delta \gamma$ scheme is mainly due to the hard-sphere approximation of $D_{s}$. It's accuracy is improved when, in place of the $\delta \gamma-D_{s}$, the simulated short-time self-diffusion coefficient is used, which is larger than the corresponding hard-sphere value. The resulting upward shift of the original $\delta \gamma$ scheme $H(q)$ leads to good overall agreement with the simulation data (see Fig. 20). The $\delta \gamma$ results for $H(q)$ shown in the figure have been obtained using an integral equation $S(q)$ whose principal peak height has been fitted to the ASD peak height for each system, by adjusting the value for the charge number $Z$. Note here that even the RY scheme underestimates to some extent the magnitude of $S\left(q_{m}\right)$ in the case of dense, deionized systems (see Fig. 4).

With increasing salt content, the undulations in $H(q)$ get smaller, reflecting a similar behavior in $S(q)$ and $g(r)$ (see Fig. 21). Both $H\left(q_{m}\right)$ and $H\left(q \gg q_{m}\right)=D_{s} / D_{0}$ decrease with increasing salinity towards the hard-sphere limiting values, and $q_{m}$ is shifted to larger values. The sedimentation coefficient $H(0)$, however, increases with increasing salt content.

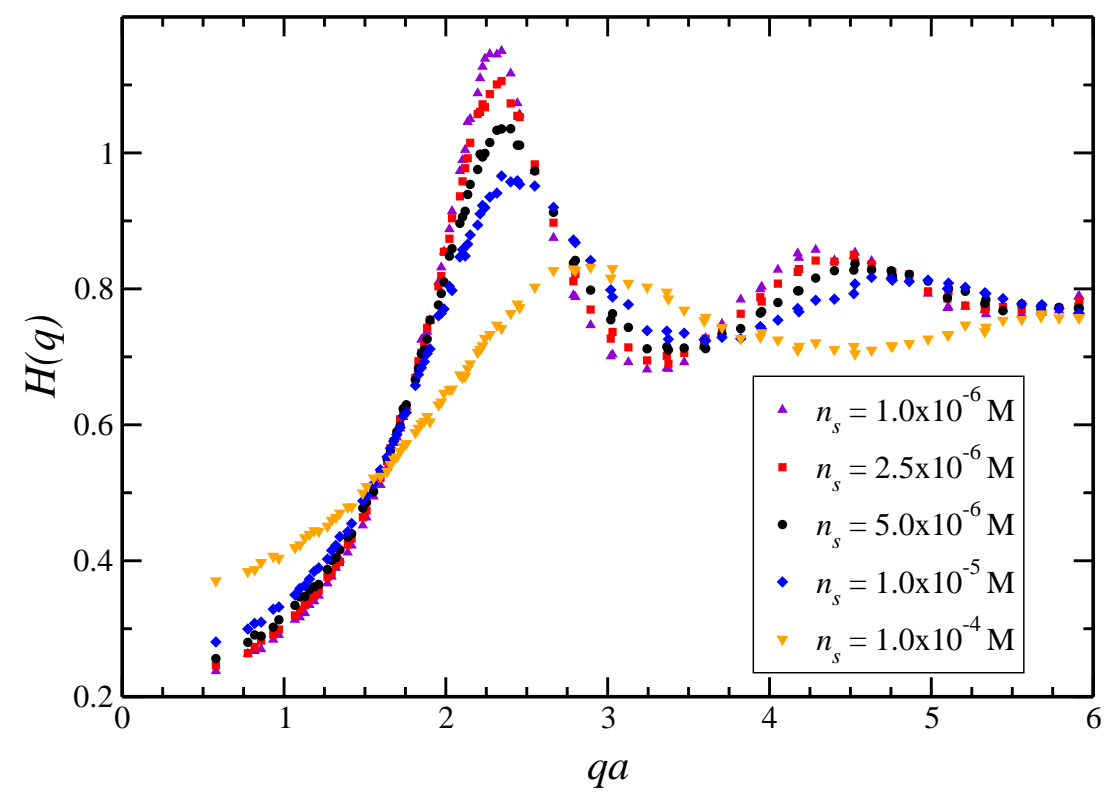

Figure 21: (Color online) ASD-simulated $H(q)$ of charged spheres at $\phi=0.15$, in dependence on the amount of added 1-1 electrolyte as indicated.

All our ASD simulation results, and analytic theory including the $\delta \gamma$ scheme, are in accord with 
the generic ordering relations, for a given $\phi$,

$$
\begin{aligned}
H^{\mathrm{CS}}\left(q_{m}\right) & >H^{\mathrm{HS}}\left(q_{m}\right), \\
D_{s}^{\mathrm{CS}} & >D_{s}^{\mathrm{HS}}, \\
U_{s}^{\mathrm{CS}} & <H^{\mathrm{HS}},
\end{aligned}
$$

valid for charge-stabilized systems that can be described by the OMF model. Here, CS and HS are the labels for charged and hard spheres, respectively. For systems with added salt, the values of these properties are always located in between the zero-salt and infinite-salt (zero-charge) limiting values. Recent short-time scattering experiments on charge-stabilized systems so different as apoferritin proteins in water [6], aqueous suspensions of fluorinated latex spheres [15], and silica spheres in DMF [96], confirm the validity of these ordering relations without any exceptions.

Depending on the system parameters under consideration, the concentration dependence of $H\left(q_{m}\right)$ can vary substantially. For weakly charged spheres, or charge-stabilized suspensions at high salinity, $H\left(q_{m}\right)$ decreases monotonically in $\phi$ similar to hard spheres. For strongly charged spheres at low salinity, on the other hand, $H\left(q_{m}\right)$ increases first sub-linearly in $\phi$ in accord with eq. (36). At some larger value of $\phi, H\left(q_{m}\right)$ passes through a maximum and declines subsequently when $\phi$ is further increased. A point in case is given by the salt-free system discussed in Fig. 20. For a thorough comparison of simulations, $\delta \gamma$ scheme calculations and experimental results on the concentration dependence of $H\left(q_{m}\right)$ we refer to [15].

The particle radius $a$ is the only static and dynamic length scale characterizing colloidal hardsphere suspensions. This leads to the approximate existence of an isobestic point, i.e., a wave number $q a \approx 4.02$ located to the right of the main structure factor peak, where both $S(q)$ and $H(q) \times D_{0} / D_{s}$, and hence also $D(q) / D_{s}$, attain the value one essentially independent of concentration. In fact, the corresponding $q$-value for $\phi=0.185$ is slightly smaller, and moves towards 4.02 with increasing $\phi$. The existence of such an isobestic point for hard spheres is shown in Fig. 22, which includes ASD simulation data for various values of $\phi$. The horizontal lines quantify the infinite- $q$ values of $S(q)$, $D(q) / D_{0}$ and $H(q)$, respectively, at the indicated volume fractions.

A suggestion due to Pusey [97] is that self-diffusion can be probed approximately at a wave number $q^{*}>q_{m}$, where $S\left(q^{*}\right)=1$. The underlying assumption is that at such a $q^{*}$ where the distinct structure factor $S_{d}(q)=S(q)-1$ is zero, also it's time-dependent generalization $S_{d}(q, t)=S(q, t)-G(q, t)$ 


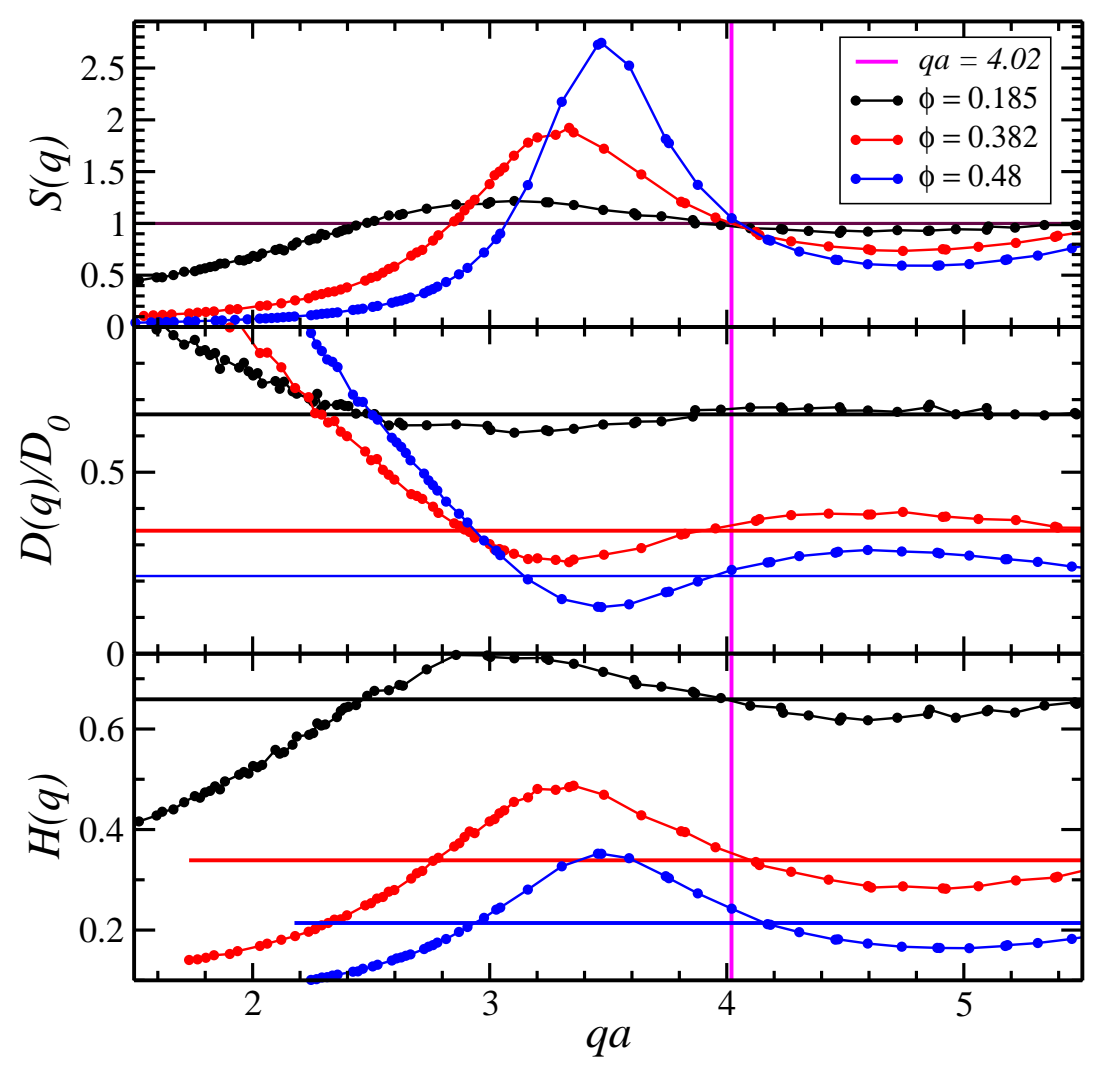

Figure 22: (Color online) The $S(q), D(q) / D_{0}$ and $H(q)$ of hard spheres suspensions at volume fractions as indicated. To a good approximation, there exists an isobestic point at $q a \approx 4.02$, where $S(q)$ and $H(q) \times$ $\left(D_{0} / D_{s}\right)$ attain the value one. Our ASD simulation data are denoted by symbols. The horizontal lines indicate the large- $q$ limiting value $D_{s} / D_{0}$ both of $D(q) / D_{0}$ and $H(q)$. 
is small, now in comparison to the self-intermediate scattering function $G(q, t)$ (with $G(q, 0)=1$ ), which describes self-diffusion [2,3]. If this assumption is valid approximately, then $D\left(q^{*}\right) / D_{0}=$ $H\left(q^{*}\right) \approx D_{s} / D_{0}$. For hard spheres, this assumption has been corroborated also by LB simulations and $\delta \gamma$ theory [5]. Our ASD simulation data on hard spheres depicted in the figure confirm this finding additionally. At all $\phi$ considered, the difference between $D_{s} / D_{0}$ and $D\left(q^{*}\right) / D_{0}=H\left(q^{*}\right)$ is less than five percent.

Unlike hard spheres, de-ionized suspensions of strongly charged spheres have at least two characteristic length scales, namely the geometric mean particle distance, $n^{-1 / 3}$, and the hydrodynamic radius $a$. Therefore, in these systems, there is no isobestic point in $S(q)$ even when it is plotted versus $q \times n^{-1 / 3}$. The non-existence of such a concentration-independent intersection point is illustrated in Fig. 23, which shows ASD results for de-ionized systems at several values of $\phi$. Even though there are no isobestic points, the ASD simulations show that the short-time self-diffusion coefficient $D_{s}$ of charged spheres can be estimated approximately, like in hard-sphere suspensions, by the $D(q)$ for a wave number $q^{*}>q_{m}$ where $S\left(q^{*}\right)=1$. For all the systems considered, and similar to the hard-sphere case, we find the difference between $D_{s} / D_{0}$ and $H\left(q^{*}\right)$ to be smaller than five percent.

\subsection{Short-time generalized Stokes-Einstein relations}

Having discussed the behavior of various short-time properties, we are in the position to examine the validity of the short-time GSE relations in Eqs. (14), (15) and (16). Fig. 24 provides a test of these relations for hard spheres. It shows our ASD simulation data for $D_{s}, D_{r}$ and $D\left(q_{m}\right)$, combined with the ASD data for $\eta_{\infty}$. The solid lines are analytic results using Eq. (27) for $\eta_{\infty}$, in combination with Eqs. (25), (21), (28), and (30) for $D_{s}$ (red line), $D_{r}$ (blue line), and $D\left(q_{m}\right) / D_{0}=H\left(q_{m}\right) / S\left(q_{m}\right)$ (black line), respectively. The orange curve is the result of combining the empirical Lionberger-Russel relations Eq. (26) for $\eta_{\infty}$ and Eq. (25) for $D_{s}$. The so-obtained analytic results are in rather good accord with the simulation data. For a GSE relation to be valid, the corresponding curve in Fig. 24 should be horizontal and equal to one. It is noted from the figure that the GSE relation involving $D_{r}$ is strongly violated for non-zero concentrations. The GSE relation for $D_{s}$, on the other hand, is less strongly violated, with $\eta_{\infty} / \eta_{0}$ lying above $D_{0} / D_{s}$ for all non-zero concentrations. The monotonic rise of $\left(\eta_{\infty} / \eta_{0}\right) \times\left(D_{s} / D_{0}\right)$ with increasing $\phi$ is overall well captured, up to $\phi=0.5$, by the first-order virial form $1+0.67 \phi$ obtained from Eqs. (20) and (23). According to second-order $\delta \gamma$ theory, the 


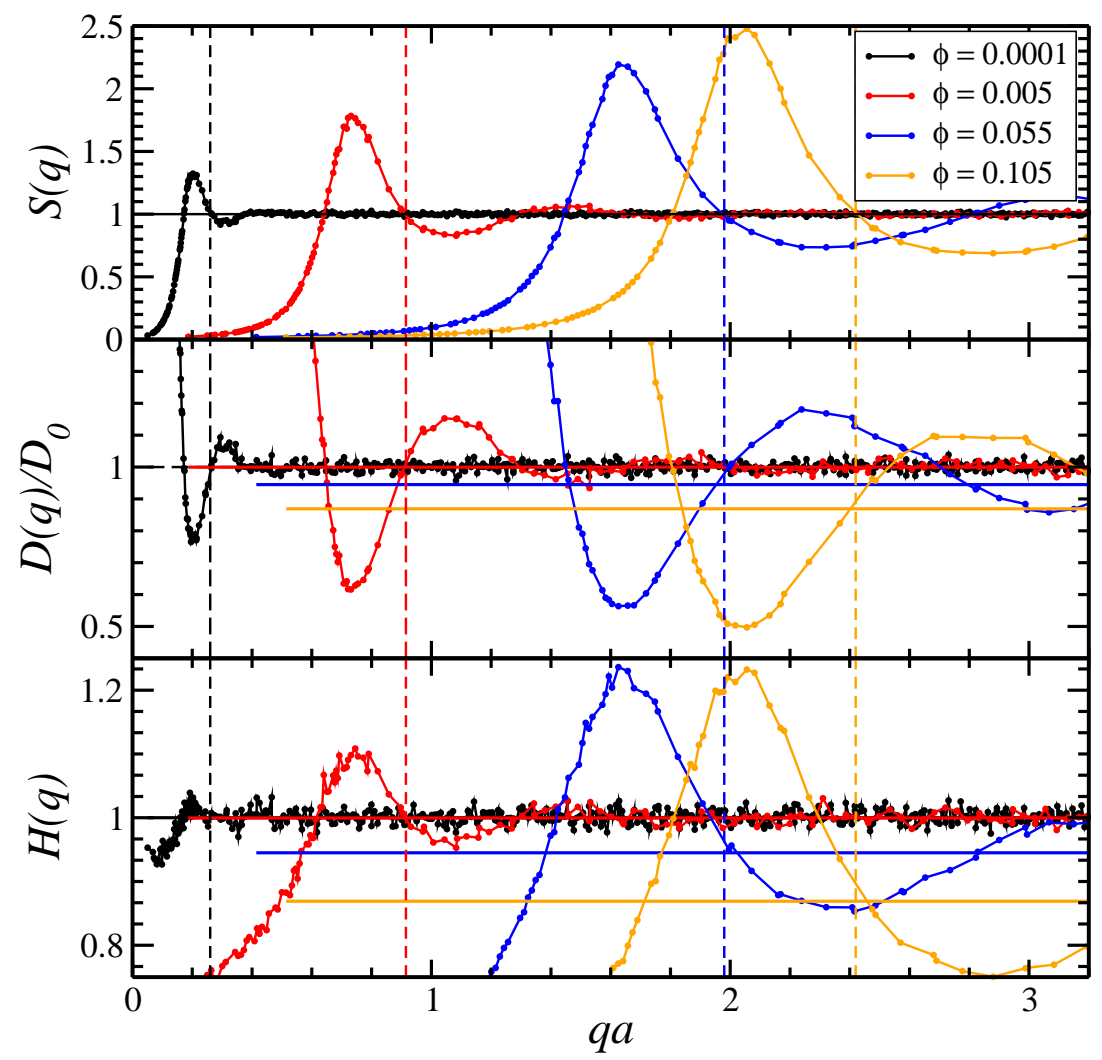

Figure 23: (Color online) The $S(q), D(q) / D_{0}$ and $H\left(q_{m}\right)$ of de-ionized suspensions of charged spheres at volume fractions as indicated. In these systems, there are no isobestic points. The ASD simulation data are denoted by the symbols. The horizontal lines are the values for $D_{s} / D_{0}$ at the indicated concentrations. 


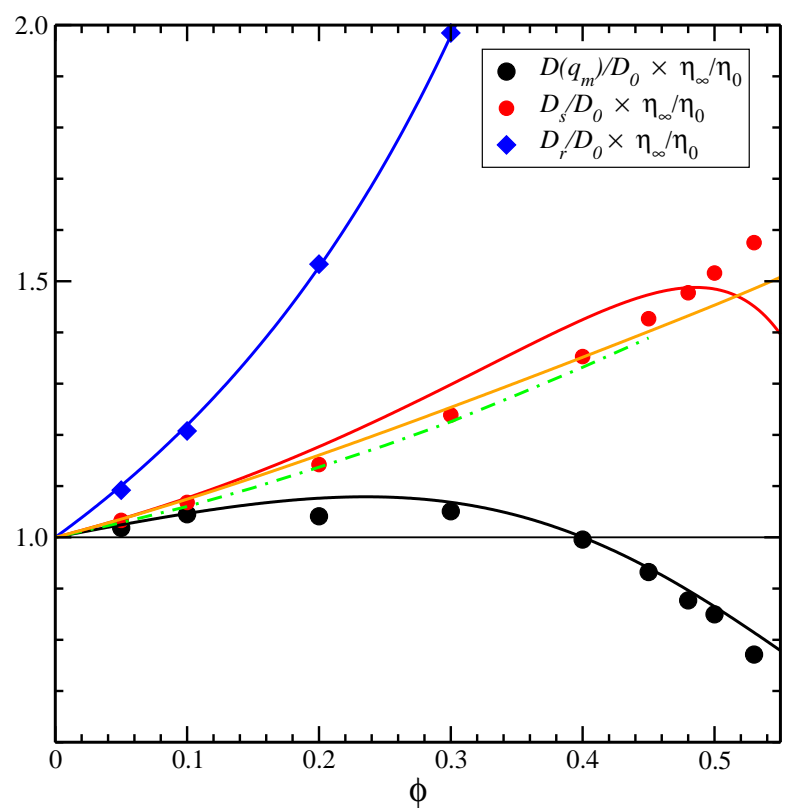

Figure 24: (Color online) ASD simulation test of the generalized SE relations in Eqs. (14), (15) and (16) for the hard-sphere case. Symbols: ASD simulation data. Solid lines: analytic results as explained in the text. Dashed-dotted line: 2nd-order $\delta \gamma$ theory prediction for the GSE relation invoking $D_{s}$ [43]. 
GSE relation for $D_{s}$ should be violated to some extent (see Fig. 4 in [43]), leading to the prediction that $D_{s} / D_{0}>\eta_{0} / \eta_{\infty}$, in qualitative accord with the simulation results. The red curve in Fig. 24 overestimates the simulation data for $D_{s} \times \eta_{\infty}$ at smaller $\phi$, and it shows a downswing for $\phi>0.5$. This is explained by the fact that, at smaller $\phi$, Eq. (25) overestimates the simulation data for $D_{s}$ to some extent, and it predicts a too strong decline of $D_{s}$ for $\phi>0.5$. The orange curve, on the other hand, is in better overall agreement with the simulation data, reflecting a fortuitous cancellation of deviations of the Lionberger-Russel expressions for $D_{s}$ and $\eta_{\infty}$ from the simulation data (see Figs. 7 and 10).

Using the SD and ASD methods, Brady et al. have computed the $\eta_{\infty}, D_{s}$ and $\eta$ of hard spheres even above the freezing point, on assuming that the dispersion can be maintained in a non-crystalline, disordered and metastable state up to random close packing. We have not include their data since, within the liquid phase regime considered in the present paper, they fully agree with our ASD data, verifying thus the correctness of the ASD scheme with Brownian forces included for the short-time dynamics of hard spheres. For concentrations $\phi>0.6$ where random closed packing is approached, and on ignoring higher-order moment contributions, Sierou and Brady [20] observe a linear scaling of $D_{s}(\phi)$ as function of the inverse high-frequency viscosity, which provides evidence that $D_{s} \propto 1 / \eta_{\infty}$ in the limit $\phi \rightarrow \phi_{\text {rcp }}$. These simulations suggest that $D_{s}$ vanishes at $\phi_{r c p}$ in a manner inversely proportional to $\eta_{\infty}$. For volume fractions below freezing, however, the physical mechanisms of selfdiffusion and viscosity are clearly distinguishable from each other, and the GSE relation for $D_{s}$ is violated to some extent, as quantified roughly by $D_{s}(\phi) / D_{0} \approx(1+0.67 \phi) \times \eta_{0} / \eta_{\infty}(\phi)$ (see also [18] and [52]).

The GSE relation for the cage diffusion coefficient, $D\left(q_{m}\right)$, of hard spheres is obeyed to a better degree of accuracy than the one for $D_{s}$. Interestingly enough, the opposite trend is found for deionized suspensions of strongly charged spheres (see Fig. 25). Here, the GSE relation for $D\left(q_{m}\right)$ is most strongly violated, for all values of $\phi$ considered, whereas the GSE for $D_{s}$ works decently well. This reflects that, different from $\eta_{\infty}, D\left(q_{m}\right)$ is highly sensitive to system parameters such as the particle charge and ionic strength, in particular through its explicit dependence on $S\left(q_{m}\right)$. The black diamonds are the ASD results for $D\left(q_{m}\right) \times \eta_{\infty}$, obtained for a less strongly correlated deionized system with system parameters identical to the ones used for the inset of Fig. 11, that characterize a system which remains fluid up to $\phi \approx 0.3$. The counterion-dominated electrostatic screening in deionized 
systems of strongly charged spheres is weak at very low $\phi$, reflected by a sharply increasing peak height $S\left(q_{m} ; \phi\right)$. This behavior of $S\left(q_{m}\right)$ taken together with the less pronounced small- $\phi$ dependence of $H\left(q_{m}\right)$, causes a steep initial decline, not resolved in the figure, of $D\left(q_{m}\right)$ and thus of $D\left(q_{m}\right) \times \eta_{\infty}$, well below it's zero-density value $D_{0}$. At larger $\phi$, however, the concentration-dependence of $D\left(q_{m}\right)$ becomes comparatively weak.

The GSE relation for $D_{r}$ in the charge-stabilized system is violated to a somewhat smaller extent than in the neutral-sphere case. We argue that the GSE relation involving $D_{r}$ is poorer than the one for $D_{s}$, owing to the shorter range of the hydrodynamic mobility associated with rotational self-diffusion. The lines in Fig. 25 are analytic results obtained from Eq. (33) for $D_{s}$ with $a_{t}=2.5$, Eq. (34) for $D_{r}$ with $a_{r}=1.3$, and Eq. (44) for $\eta_{\infty}$. The decay in the analytic curve for the GSE relation involving $D_{s}$ for $\phi>0.15$ reflects that Eq. (33) ceases to be valid at higher concentrations. There is no simple analytic expression available, to the best of our knowledge, giving the peak height $S\left(q_{m}\right)$ of charged spheres. Furthermore, and as noted earlier, the parameter $p_{m}$ in Eq. (36) is sensitive to the values of $Z$ and $\kappa a$. Therefore, no analytic result can be shown in the figure describing the concentration dependence of $D\left(q_{m}\right) \times \eta_{\infty}$.

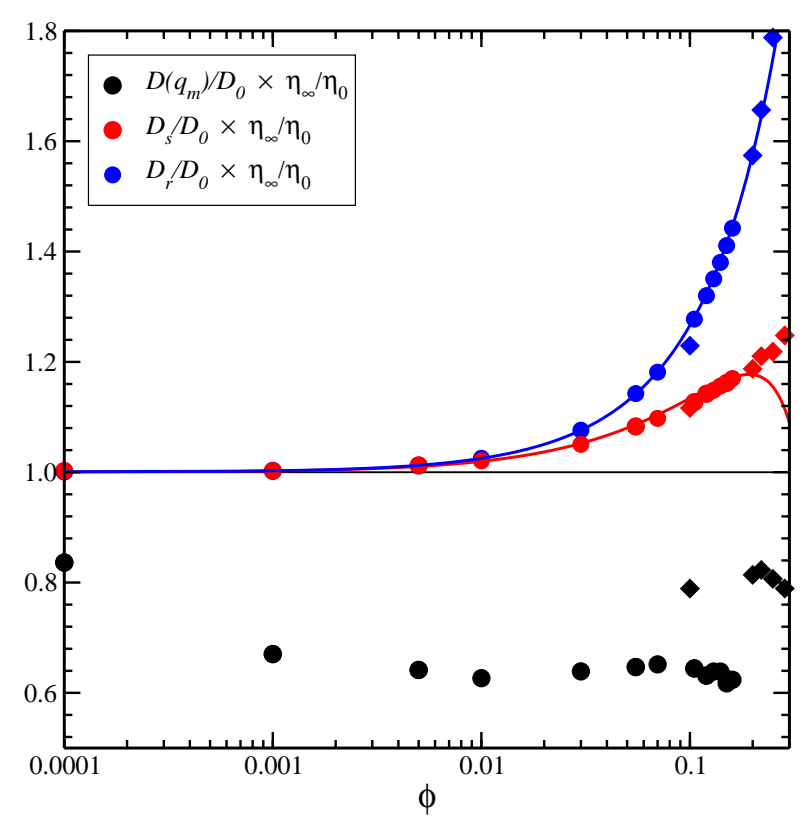

Figure 25: (Color online) ASD simulation test of the GSE relations for de-ionized charge-stabilized suspensions. The solid lines are based on theoretical expressionns for $D_{s}, D_{r}$ and $\eta_{\infty}$ as explained in the text. The GSE relation between $D_{s}$ and $\eta_{\infty}$ is decently well obeyed, similar to the HS case. In contrast to hard spheres, the GSE relation for $D\left(q_{m}\right)$ is strongly violated. 
We conclude our discussion of short-time GSE relations by noting that the ordering relation $D_{s}>$ $k_{B} T /\left(6 \pi \eta_{\infty} a\right)$ is valid both for de-ionized suspensions and neutral hard spheres, in agreement with experiments of Bergenholtz et al., where $\eta_{\infty}$ and $D_{s}$ have been measured for various ionic strengths and concentrations [76].

\section{CONCLUDING REMARKS}

This work includes the first comprehensive (accelerated) SD computer simulation study on the shorttime dynamic properties of charge-stabilized suspensions. Using an extension of the ASD simulation method to the OMF model of charged colloidal spheres, numerous short-time quantities have been calculated including collective and self-diffusion coefficients, sedimentation coefficient, high-frequency limiting viscosity, and hydrodynamic functions. The short-time dynamics of colloidal hard spheres has been part of this study in order to highlight the qualitative differences between low-salinity chargestabilized suspensions, and hard-sphere-like systems which have a high salt content. The range of validity, and the degree of accuracy, of various analytic short-time expressions have been explored through comparison with our ASD simulation data in the case of charged spheres and, for neutral hard spheres, also with earlier SD and ASD simulation results of Brady and coworkers, and LB simulation data of Ladd and Behrend et al. The assessment of the quality of these handy-to-use analytic expressions is a necessary prerequisite for the experimentalist who wishes to use these expressions for data interpretation, and in any theory on colloidal long-time dynamics where short-time properties are the basic input.

For hard spheres, in particular, we have assessed the concentration range where the currently available truncated virial expansion results by Cichocki and coworkers are valid. Moreover, we have shown that the expressions in Eqs. (28), (30) and (27) provide excellent descriptions of $H\left(q_{m}\right), D\left(q_{m}\right)$ and $\eta_{\infty}$ in the whole fluid regime.

For de-ionized suspensions characterized by long-range repulsive forces, simple physical arguments based on the dominance of far-field HI in these systems, and the $\phi^{-1 / 3}$ concentration scaling of the next-neighbor shell diameter, have led to short-time expressions showing a non-linear or even fractal concentration dependence. The present ASD simulation study has allowed us to quantify the accuracy of these expressions. We have found that most of them apply quantitatively in a restricted $\phi$-range only, as one can expect from the assumptions made in their derivation. Eqs. (34) and (44) 
for $D_{r}$ and $\eta_{\infty}$, however, are valid to good accuracy even for fluid systems with very strong particle correlations. For low-salt systems, the simulations confirm the values of the exponents in the Eqs. (33)-(36) which are independent of the details of the interaction parameters.

The ASD simulation study of isobestic points and, more specifically, of wave numbers where $S(q)=1$, has allowed us to quantify the experimental error made in determining $D_{s}$ through dynamic light scattering measurements at such points. This is of relevance for numerous colloidal systems where the large- $q$ regime is not accessible experimentally, and where partial index matching techniques are not applicable.

Various short-time GSE relations have been examined. We find that rotational self-diffusion and, to a lesser extent, translational self-diffusion is larger than predicted by the corresponding GSE relations. The amount by which a GSE relation is violated depends sensitively on the range of the electrostatic interaction. For example, the GSE relation for the cage diffusion coefficient is valid in the case of hard spheres to decently good accuracy, but is strongly violated in de-ionized suspensions. The general trends in the behavior of $D_{s}$ and $\eta_{\infty}$, as predicted by the ASD simulations, are in agreement with the experimental findings on charge-stabilized dispersions.

Furthermore, our simulations show that the $\delta \gamma$ theory of Beenakker and Mazur, initially applied to hard spheres only, remains useful in the case of charged spheres, where it can be used for short-time calculations on a semi-quantitative level of accuracy. In calculating $H(q)$, the accuracy of the zerothorder $\delta \gamma$ theory is improved when combined with a more accurate calculation of the self-part $D_{s}$ based, e.g., on a rooted cluster approximation for charged spheres. The $\delta \gamma$ theory is particularly suited for systems of intermediate salt content, where no simple short-time analytic expressions are available, and where a high data throughput is required which makes simulation calculations prohibitively expensive.

The basis of our ASD simulation study has been the OMF model describing dressed spherical macroions. To date this is the most widely used model for charge-stabilized suspensions of particles with negligibly small dispersion attractions. The OMF model has been extended in various ways to account for macroion charge-renormalization effects arising from the quasi-condensation of counterions near strongly charged colloid surfaces, and for electrokinetic effects caused by the non-instantaneous dynamic response of the electrolyte atmosphere formed around each colloidal particle. Recent calculations based on a mode coupling scheme applied to the primitive model that includes hydrodynamic interactions between all ionic species show that the electrokinetic influence on the colloidal self-diffusion 
is quite small, and ceases with increasing volume fraction [11]. The effect of the electrolyte dynamics is somewhat stronger when collective diffusion is considered, where it can cause a reduction in $D_{c}$ by several percent [6]. This reduction is most pronounced at low salinity, but is becomes weaker with increasing colloid-microion size ratio. However, the very small values of $H(q)$ purportedly measured in recent experiments on certain de-ionized suspensions by Grübel, Robert and coworkers [34,48,98], can not be explained by this secondary electrokinetic effect which diminishes at large macroion-microion size ratios. These authors find values of $H\left(q_{m}\right)$ that are roughly by a factor of two smaller than the peak heights obtained from our simulation calculations $[6,15]$, with the latter being consistent with experimental data for $H(q), D_{c}, U_{s}$ and $D_{s}$ obtained by other groups [49, 50,71,75,76,99].

The present paper was concerned with monodisperse suspensions of charged colloidal spheres with negligible attractive forces. In future extensions of this simulation work, we will address the short-time diffusion and rheology of particles with a non-negligible attractive interaction part such as globular lysozyme proteins, and we will study the dynamics in binary colloidal mixtures.

\section{Acknowledgments}

This work has been supported by the Deutsche Forschungsgemeinschaft (SFB-TR6), and by CONICET, FONCYT (PICT 2005-33691) and SECYT-UNC, Argentina. A.J. Banchio expresses his deep gratitude to Prof. John Brady, who is co-developer of the ASD code for Brownian spheres. Without his contribution, the present simulation work would not have been possible. We gratefully acknowledge discussions with J.K.G. Dhont, J. Gapinski, Ch. Gögelein, M. McPhie, and A. Patkowski.

\section{References}

[1] W.B. Russel, D.A. Saville and W.R. Schowalter, Colloidal Dispersions (Cambridge University Press, Cambridge, 1989).

[2] P.N. Pusey, in Liquids, Freezing and the Glass Transition, edited by J.-P. Hansen, D. Levesque and J. Zinn-Justin (Elsevier, Amsterdam, 1991) pp. 763-942.

[3] G. Nägele, Phys. Rep. 272, 215 (1996).

[4] J.K.G. Dhont, An Introduction to Dynamics of Colloids (Elsevier, Amsterdam, 1996). 
[5] P. N. Segrè, O. P. Behrend and P. N. Pusey, Phys. Rev. E 52, 5070 (1995).

[6] J. Gapinski, A. Wilk, A. Patkowski, W. Häussler, A.J. Banchio, R. Pecora and G. Nägele, J. Chem. Phys. 123, 1054708 (2005).

[7] A. Evilevitch, V. Lobaskin, U. Olsson, P. Linse and P. Schurtenberger, Langmuir 17, 1043 (2001).

[8] G. Nägele and P. Baur, Europhys. Lett. 38, 557 (1997).

[9] K. Zahn, J.M. Méndez-Alcaraz and G. Maret, Phys. Rev. Lett. 79, 175 (1997).

[10] W. Härtl, J. Wagner, Ch. Beck, F. Gierschner and R. Hempelmann, J. Phys.: Condens. Matter 12, A287 (2000).

[11] M.G. McPhie and G. Nägele, J. Chem. Phys. 127, 034906 (2007).

[12] M.G. McPhie and G. Nägele, J. Phys.: Condens. Matter 16, S4021 (2004).

[13] A.J. Banchio and J.F. Brady, J. Chem. Phys. 118, 10323 (2003)

[14] A.J. Banchio, J. Gapinski, A. Patkowski, W. Häussler, A. Fluerasu, S. Saccana, P. Holmqvist, G. Meier, M.P. Lettinga and G. Nägele, Phys. Rev. Lett. 96, 138303 (2006).

[15] J. Gapinski, A. Patkowski, A.J. Banchio, P. Holmqvist, G. Meier, M.P. Lettinga and G. Nägele, J. Chem. Phys. 126, 104905 (2007).

[16] R.J. Philips, J.F. Brady and G. Bossis, Phys. Fluids 31, 3462 (1988).

[17] J.F. Brady, R.J. Phillips, J.C. Lester and G. Bossis, J. Fluid Mech. 195, 257 (1988).

[18] J.F. Brady, Curr. Opin. Colloid Interface Sci. 1, 472 (1996).

[19] D.R. Ross and J.F. Brady, J. Fluid Mech. 407, 167 (2000).

[20] A. Sierou and J.F. Brady, J. Fluid Mech. 448, 115 (2001).

[21] A.J.C. Ladd, J. Chem. Phys. 93, 3484 (1990).

[22] A.J.C. Ladd, H. Gang, J.X. Zhu and D.A. Weitz, Phys. Rev. E 52, 6550 (1995).

[23] O.P. Behrend, Phys. Rev. E 52, 1164 (1995). 
[24] O.P. Behrend, Ph.D. thesis, University of Edinburgh (1995).

[25] M.H.J. Hagen, D. Frenkel and C.P. Lowe, Physica A 272376 (1999).

[26] M.W. Heemels, M.H.J. Hagen and C.P. Lowe, J. Comput. Phys. 16448 (2000).

[27] A.J.C. Ladd, J. Fluid Mech. 271, 285 (1994), 271, 311 (1994).

[28] I. Snook, W. van Megen and R.J.A. Tough, J. Chem. Phys. 78, 5825 (1983).

[29] W. van Megen and I. Snook, J. Chem. Soc., Faraday Trans. 2 80, 383 (1984).

[30] W. van Megen and I. Snook, J. Chem. Phys. 88, 1185 (1987).

[31] S. Walrand, L. Belloni and M. Drifford, J. Physique 47, 1565 (1986).

[32] C.W.J. Beenakker and P. Mazur, Physica 126 A, 349 (1984).

[33] J.F. Brady and L.J. Durlofsky, Phys. Fluids 31, 717 (1988).

[34] D.O. Riese, G.H. Wegdam, W.L. Vos, R. Sprik, D. Fenistein, J.H.H. Bongaerts and G. Grübel, Phys. Rev. Lett. 85, 5460 (2000).

[35] B. Cichocki, M.L. Ekiel-Jezewska and E. Wajnryb, J. Chem. Phys. 111, 3265 (1999).

[36] B. Cichocki, M.L. Ekiel-Jezewska, P. Szymczak and E. Wajnryb, J. Chem. Phys. 117, 1231 (2002).

[37] B. Cichocki, M.L. Ekiel-Jezewska and E. Wajnryb, J. Chem. Phys. 119, 606 (2003).

[38] M. Watzlawek and G. Nägele, Phys. Rev. E 56, 1258 (1997).

[39] H. Zhang and G. Nägele, J. Chem. Phys. 117, 5908 (2002).

[40] G.H. Koenderink, H. Zhang, D.G.A.L. Aarts, M.P. Lettinga, A.P. Philipse and G. Nägele, Faraday Discuss. 123335 (2002).

[41] C.W.J. Beenakker and P. Mazur, Phys. Lett. A 98, 22 (1983).

[42] C.W.J. Beenakker, Ph.D. thesis, University of Leiden (1984).

[43] C.W.J. Beenakker, Physica A 128, 48 (1984). 
[44] R.L. Treloar and A.J. Masters, Mol. Phys. 67, 1273 (1989).

[45] U. Genz and R. Klein, Physica A 171, 26 (1991).

[46] G. Nägele, B. Steininger, U. Genz and R. Klein, Physica Scripta T 55, 119 (1994).

[47] G. Nägele, B. Mandl and R. Klein, Progr Colloid Polym Sci 98, 117 (1995).

[48] A. Robert, Ph.D. thesis, Université Joseph Fourier, Grenoble, France (2001).

[49] L.F. Rojas, R. Vavrin, C. Urban, J. Kohlbrecher, A. Stradner, F. Scheffold and P. Schurtenberger, Faraday Discuss 123, 385 (2003).

[50] L.F. Rojas, Ph.D. thesis, University of Fribourg, Switzerland (2004).

[51] E.G.D. Cohen, R. Verberg and I.M. de Schepper, Physica A 251, 251 (1998).

[52] A.J. Banchio, G. Nägele and J. Bergenholtz, J. Chem. Phys. 111, 8721 (1999).

[53] A.J. Banchio, J. Bergenholtz and G. Nägele, Phys. Rev. Lett. 82, 1792 (1999).

[54] M. Fuchs and M.R. Mayr, Phys. Rev. E 60, 5742 (1999).

[55] A.R. Denton, Phys. Rev. E 62, 3855 (2000).

[56] J.W. Verwey and J.T.G. Overbeek, Theory of the Stability of Lyotropic Colloids (Elsevier. Amsterdam, 1948).

[57] L. Belloni, J. Chem. Phys. 85, 519 (1986).

[58] W.B. Russel and D.W. Benzing, J. Colloid Interface Sci. 83, 163 (1981).

[59] E. Trizac, L. Bocquet, M. Aubouy and H.H. von Grünberg, Langmuir 19, 4027 (2003).

[60] B. Beresford-Smith, D.Y. Chan abd D.J. Mitchell, J. Colloid Interface Sci. 105, 216 (1984).

[61] A. Diehl and Y. Levin, J. Chem. Phys. 121, 12100 (2004).

[62] W.L. Hsin, T.Y. Wang, Y.J. Sheng and H.K. Tsao, J. Chem. Phys. 121, 5494 (2004).

[63] S. Pianegonda, E. Trizac and Y. Levin, J. Chem. Phys. 126, 014702 (2007). 
[64] A.P. Hynninen and M. Dijkstra, J. Chem. Phys. 123, 244902 (2005).

[65] T. Kreer, J. Horbach and A. Chatterji, Phys. Rev. E 74, 021401 (2006).

[66] J.P. Hansen and I.R. McDonald, Theory of Simple Liquids, (2nd Edition, Academic Press, London, 1986).

[67] F.J. Rogers and D.A. Young, Phys. Rev. A 30, 999 (1984).

[68] J.P. Hansen and J.B. Hayter, Mol. Phys. 46651 (1982).

[69] G. Nägele, The Physics of Colloidal Soft Matter, Lecture Notes 14, Institute of Fundamental Technological Research (Polish Academy of Sciences publication, Warsaw, 2004).

[70] R.B. Jones and P.N. Pusey, Annu. Rev. Phys. Chem. 42, 137 (1991).

[71] J.K. Phalakornkul, A.P. Gast, R. Pecora, G. Nägele, A. Ferrante, B. Mandl-Steininger, and R. Klein, Phys. Rev. E 54, 661 (1996).

[72] D.M.E. Thies-Weesie, A.P. Philipse, G. Nägele, B. Steininger and R. Klein, J. Colloid Interface Sci. 176, 43 (1995).

[73] E. Wajnryb, P. Szymczak and B. Cichocki, Physica A 335, 339 (2004).

[74] V. Degiorgio, R. Piazza and R.B. Jones, Phys. Rev. E 52, 2707 (1995).

[75] E. Overbeck, C. Sinn and M. Watzlawek, Phys. Rev. E 60, 1936 (1999).

[76] J. Bergenholtz, F.M. Horn, W. Richtering, N. Willenbacher and N.J. Wagner, Phys. Rev. E 58, R4088 (1998).

[77] G. Nägele, J. Phys.: Condens. Matter 15, S407 (2003).

[78] J. Zhang and X.Y. Liu, J. Chem. Phys. 119, 10972 (2003).

[79] B. Cichocki and B.U. Felderhof, J. Chem. Phys. 93, 4427 (1990).

[80] K.F. Seefeldt and M.J. Solomon, Phys. Rev. E 67, 050402(R) (2003).

[81] A.T.J.M. Woutersen, J. Mellema, C. Blom and C.G. de Kruif, J. Chem. Phys. 101, 542 (1994). 
[82] R.A. Lionberger and W.B. Russel, J. Rheology 38, 1885 (1994).

[83] H.J.H. Clercx and P.P.J.M. Schram, Phys. Rev. A 45, 860 (1992).

[84] A.J. Banchio, G. Nägele and J. Bergenholtz, J. Chem. Phys. 113, 3381 (2000).

[85] P.N. Segrè, S.P. Meeker, P.N. Pusey and W.C.K. Poon, Phys. Rev. Lett. 74, 1250 (1995).

[86] F.-U. Tao, Y. Song and E.A. Mason, Phys. Rev. A 46, 8007 (1992).

[87] M. Watzlawek and G. Nägele, J. Colloid Interface Sci. 214, 170 (1999).

[88] G. Nägele, O. Kellerbauer, R. Krause and R. Klein, Phys. Rev. E 47, 2562 (1993).

[89] W.B. Russel, J. Fluid Mech. 85, 209 (1978).

[90] W. Härtl, Ch. Beck and R. Hempelmann, J. Chem. Phys. 110, 7070 (1999).

[91] M.S. Wertheim, J. Math. Phys. 5, 643 (1964).

[92] F.M. Horn, W. Richtering, J. Bergenholtz, N. Willenbacher and N.J. Wagner, J. Colloid Interface Sci. 225, 166 (2000).

[93] D.M. Heyes and H. Sigurgeirsson, J. Rheol. 48, 223 (2004).

[94] H. Hasimoto, J. Fluid Mech. 5, 317 (1959).

[95] P.G. Saffman, Stud. Appl. Math. 52, 115 (1973).

[96] J. Gapinski, A. Patkowski, A.J. Banchio, G. Nägele et al., in preparation.

[97] P.N. Pusey, J. Phys. A 11, 119 (1978).

[98] T. Autenrieth, A. Robert, J. Wagner and G. Grübel, J. Appl. Cryst. 40, s250 (2007).

[99] A.P. Philipse and A. Vrij, J. Chem. Phys. 88, 6459 (1988) 


\section{Figure Captions}

Fig. 1 (Color online) Static structure factor of a hard-sphere suspension at various volume fractions $\phi$ as indicated. Comparison between MD simulation data and Rogers-Young (RY), Percus-Yevick (PY) and Verlet-Weiss corrected Percus-Yevick (PY-VW) integral equation schemes.

Fig. 2 (Color online) Peak value, $S\left(q_{m}\right)$, of the hard-sphere static structure factor. MD simulation data (symbols) are compared with PY calculations and the PY-VW fitting formula in Eq. (30).

Fig. 3 (Color online) Hard-sphere radial distribution function corresponding to Fig. 1. The curves for $\phi=0.40$ and 0.20 are shifted to the right by $a$ and $2 a$, respectively.

Fig. 4 (Color online) Static structure factor of a de-ionized charge-stabilized suspension at volume fractions as indicated. The MC simulation data (symbols) are compared with Rogers-Young calculations (solid lines) using identical system parameters, i.e., $Z=100, L_{B}=5.62 \mathrm{~nm}$ and $n_{s}=0$.

Fig. 5 (Color online) Concentration dependence of the peak position, $q_{m}$, of the static structure factor of de-ionized suspension in units of the particle radius. The green diamonds are the RMSA result for $n_{s}=0$. Blue circles: MC data for $n_{s}=0$; Black circles: MC result for a collection of systems with varying amount of added salt. Red circles: MD data for a system at $\phi=0.15$ with $n_{s}$ varying from $1 \times 10^{-6}$ to $1 \times 10^{-4} \mathrm{M}$. Additionally shown is the peak-position wave number of hard spheres, as predicted by the Verlet-Weiss corrected PY scheme.

Fig. 6 (Color online) Translational short-time self-diffusion coefficient of hard spheres versus volume fraction $\phi$. Comparison of the ASD and force multipole simulation data with the zeroth order and second order $\delta \gamma$ theory prediction, the 2nd order virial expansion result of Cichocki et al., the semiempirical expression of Lionberger and Russel in Eq. (25), and the experimental data of Segrè et al. [5].

Fig. 7 (Color online) Translational short-time self-diffusion coefficient of charged spheres in a saltfree suspension (labelled by CS) versus the hard-sphere result (HS). The symbols are our ASD simulation results. Green diamonds: hard spheres; blue circles: de-ionized suspension; red circles: transition 
from a de-ionized system, at $\phi=0.15$, to a hard-sphere-like system on increasing $n_{s}$ from $1 \times 10^{-6}$ to $1 \times 10^{-4} \mathrm{M}$; black circles: collection of systems with varying amount of added salt.

Fig. 8 (Color online) Short-time rotational self-diffusion coefficient of hard spheres versus volume fraction. The symbols are our ASD results and correspond to the ones in Figs. 6 and 7. The ASD data are compared with Lattice-Boltzmann simulation data of Hagen et al. [25], the truncated virial expansion (in Eq. (21)), and the experimental data of Degiorgio et al. [74]. The 2nd-order virial expression remains valid up to $\phi=0.45$.

Fig. 9 (Color online) Short-time rotational self-diffusion coefficient of a de-ionized suspension of charged spheres (CS), in comparison to the $D_{r}$ of neutral spheres (HS). The symbols are our ASD simulation results, with same symbols and color coding as in Fig. 7. The quadratic scaling form in Eq. (34), which accounts for far-field 3-body HI corrections, remains valid up to remarkably large $\phi$. The $D_{r}$ for systems with added salt is bounded from above and below, respectively, by the two limiting curves describing de-ionized charged-sphere and neutral hard-sphere systems.

Fig. 10 (Color online) High-frequency limiting viscosity, $\eta_{\infty}$, of colloidal hard spheres versus $\phi$. Displayed are our ASD simulation data in comparison with the force multipole simulation data of Ladd [21], the third-order truncated virial expansion result in Eq. (23), the semi-empirical LionbergerRussel expression in Eq. (26), the simulation fitting formula of Ladd in Eq. (27), the modified Saito expression of Cichocki et al. in Eq. (45), and the 2nd order $\delta \gamma$-PY result taken from [43].

Fig. 11 (Color online) High-frequency limiting viscosity of two de-ionized suspensions of charged spheres (Circles: $Z=100, a=100 \mathrm{~nm}, L_{B}=5.62 \mathrm{~nm}, n_{s}=0$; diamonds: $Z=70, a=25 \mathrm{~nm}$, $\left.L_{B}=0.71 \mathrm{~nm}, n_{s}=0\right)$. The ASD simulation data are overall well described by Eq. (44) which derives from a schematic model for $g(r)$ using the leading-order far-field HI contribution. For an expanded view of the differences, the inset shows the excess short-time viscosity.

Fig. 12 (Color online) Short-time sedimentation coefficient, $U_{s} / U_{0}$, of a homogeneous hard-sphere suspension. The 2nd-order virial and Rotne-Prager approximation results, and the zeroth-order $\delta \gamma$ 
scheme prediction are compared with the accurate computer simulation results of Ladd [21], and LB simulations of Segrè et al. [5]. Note here the strong difference between $\eta_{0} / \eta_{\infty}(\phi)$ and $\eta_{0} / \eta(\phi)$.

Fig. 13 (Color online) Sedimentation coefficient, $U_{s} / U_{0}$, of a de-ionized charge-stabilized suspension versus $\phi$. Experimental data are taken from [50], and compared with the scaling form in Eq. (35) using $a_{s}=1.8$. The sedimentation coefficient of hard spheres as described by Eq. (22) is shown for comparison.

Fig. 14 (Color online) Short-time collective diffusion coefficient, $D_{c}=D(q \rightarrow 0)$, of hard spheres. Comparison between the simulation data of Ladd [21], obtained from dividing the simulated $U_{s} / U_{0}$ by $S_{\mathrm{CS}}(0), \mathrm{LB}$ simulation data and dynamic light scattering data of Segrè et al. [5], zeroth-order $\delta \gamma$ theory prediction, and the 2nd-order virial result in Eq. (24).

Fig. 15 (Color online) Uncorrected ASD-simulated hydrodynamic function, $H_{N}(q)$, of a chargestabilized suspension (lines) with $\phi=0.123, Z=1400, a=82.5 \mathrm{~nm}$, and $L_{B}=0.71 \mathrm{~nm}$, for various numbers $N$ of simulated spheres as indicated. The finite-size corrected functions (symbols), obtained using Eq. (17), collapse on a single curve that is identified as $H(q)$.

Fig. 16 (Color online) ASD simulation results for the hydrodynamic function of hard spheres in comparison with the $\delta \gamma$ theory predictions.

Fig. 17 (Color online) ASD simulation predictions for the short-time diffusion function of hard spheres in comparison with the $\delta \gamma$ theory predictions.

Fig. 18 (Color online) Peak height, $H\left(q_{m}\right)$, of the hydrodynamic function of hard spheres. ASD simulation data are compared with the LB predictions of Behrend et al. [5], the $\delta \gamma$ theory with PY input, experimental data of Segrè et al [5], and the empirical expression $1-1.35 \phi$ in Eq. (28).

Fig. 19 (Color online) Cage diffusion coefficient, $D\left(q_{m}\right)$, of colloidal hard spheres versus volume fraction. The analytic expression for $D\left(q_{m}\right)$, defined by the ratio of Eqs. (28) and (30), is tested against 
ASD simulation data, and the experimental data of Segrè et al. [5].

Fig. 20 (Color online) ASD-simulated hydrodynamic function $H(q)$ of charged spheres at zero added salt content, in dependence on $\phi$. The $\delta \gamma$ theory result (dashed lines) and the self-part corrected $\delta \gamma$ theory result (solid lines) are included for comparison.

Fig. 21 (Color online) ASD-simulated $H(q)$ of charged spheres at $\phi=0.15$, in dependence on the amount of added 1-1 electrolyte as indicated.

Fig. 22 (Color online) The $S(q), D(q) / D_{0}$ and $H(q)$ of hard spheres suspensions at volume fractions as indicated. To a good approximation, there exists an isobestic point at $q a \approx 4.02$, where $S(q)$ and $H(q) \times\left(D_{0} / D_{s}\right)$ attain the value one. Our ASD simulation data are denoted by symbols. The horizontal lines indicate the large- $q$ limiting value $D_{s} / D_{0}$ both of $D(q) / D_{0}$ and $H(q)$.

Fig. 23 (Color online) The $S(q), D(q) / D_{0}$ and $H\left(q_{m}\right)$ of de-ionized suspensions of charged spheres at volume fractions as indicated. In these systems, there are no isobestic points. The ASD simulation data are denoted by the symbols. The horizontal lines are the values for $D_{s} / D_{0}$ at the indicated concentrations.

Fig. 24 (Color online) ASD simulation test of the generalized SE relations in Eqs. (14), (15) and (16) for the hard-sphere case. Symbols: ASD simulation data. Solid lines: analytic results as explained in the text. Dashed-dotted line: 2nd-order $\delta \gamma$ theory prediction for the GSE relation invoking $D_{s}$ [43].

Fig. 25 (Color online) ASD simulation test of the GSE relations for de-ionized charge-stabilized suspensions. The solid lines are based on theoretical expressionns for $D_{s}, D_{r}$ and $\eta_{\infty}$ as explained in the text. The GSE relation between $D_{s}$ and $\eta_{\infty}$ is decently well obeyed, similar to the HS case. In contrast to hard spheres, the GSE relation for $D\left(q_{m}\right)$ is strongly violated. 


\section{Figures}

Banchio and Nägele - Fig. 1

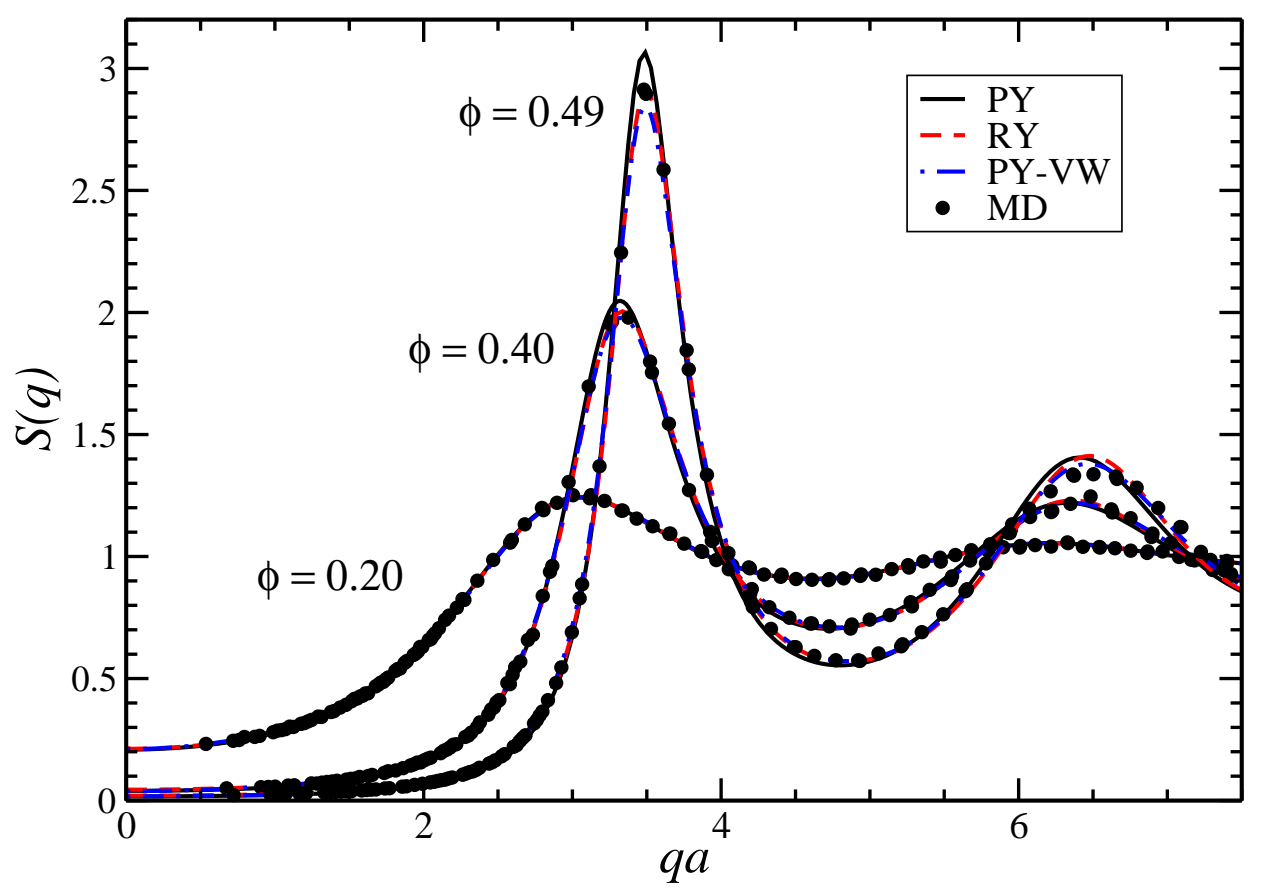


Banchio and Nägele - Fig. 2

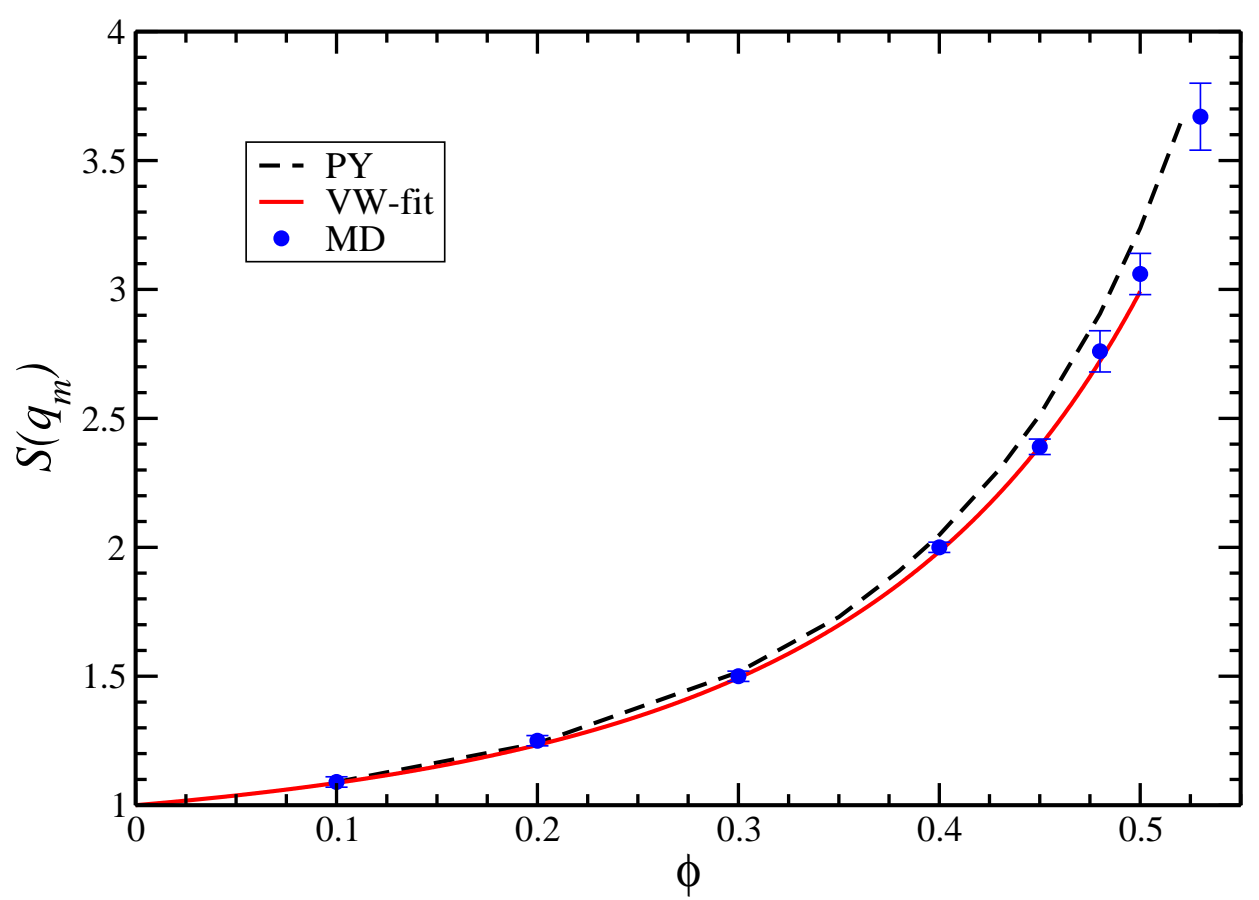


Banchio and Nägele - Fig. 3

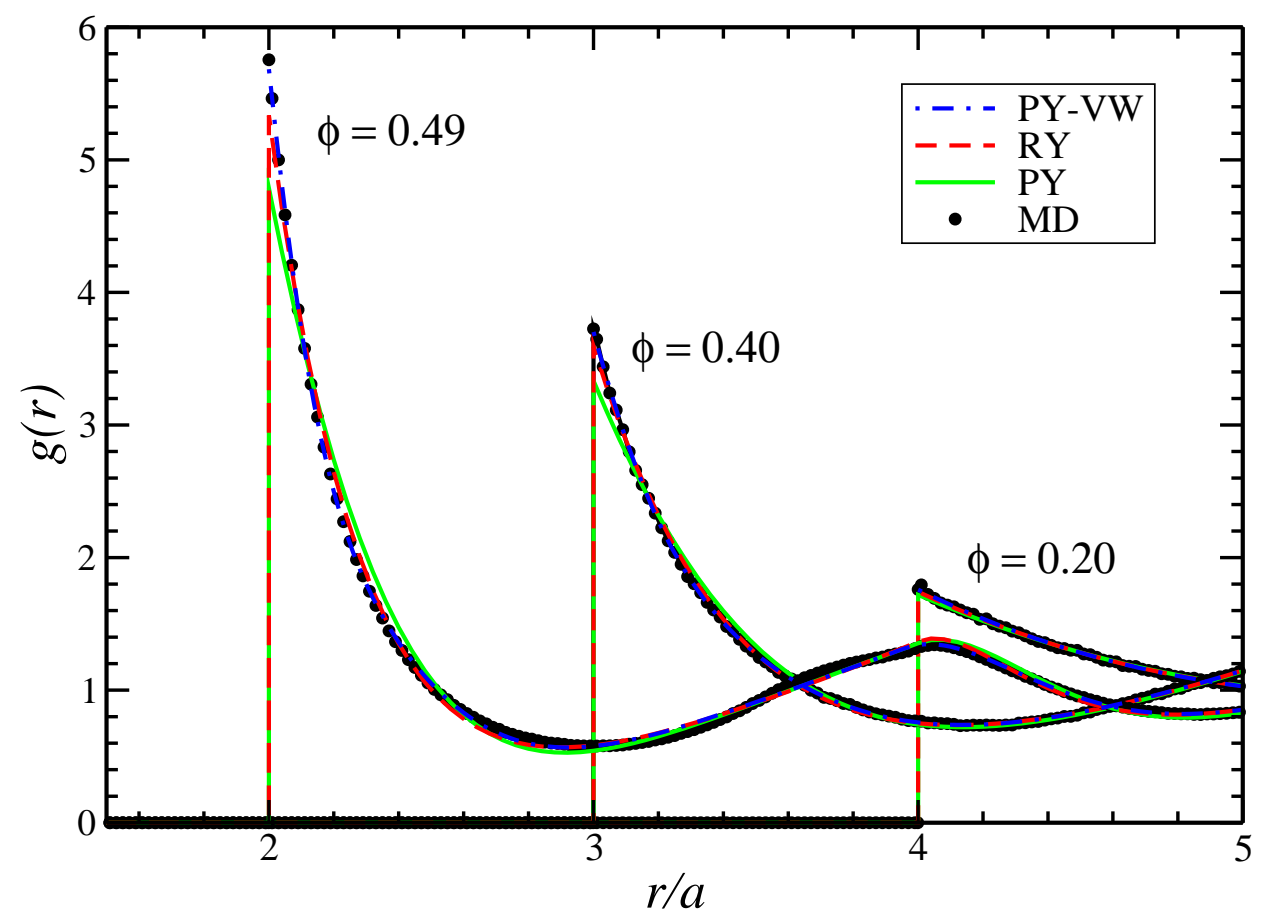


Banchio and Nägele - Fig. 4

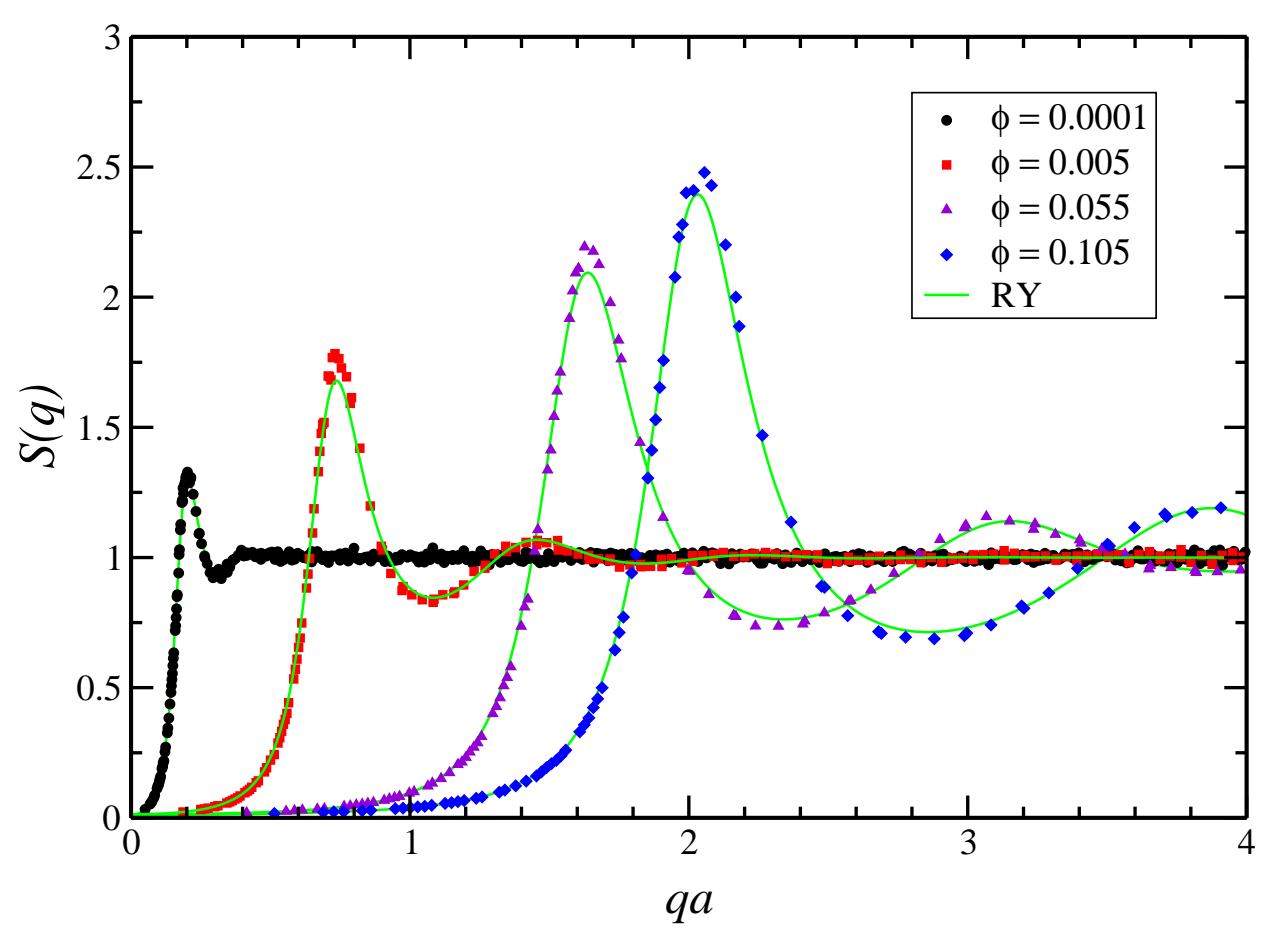


Banchio and Nägele - Fig. 5

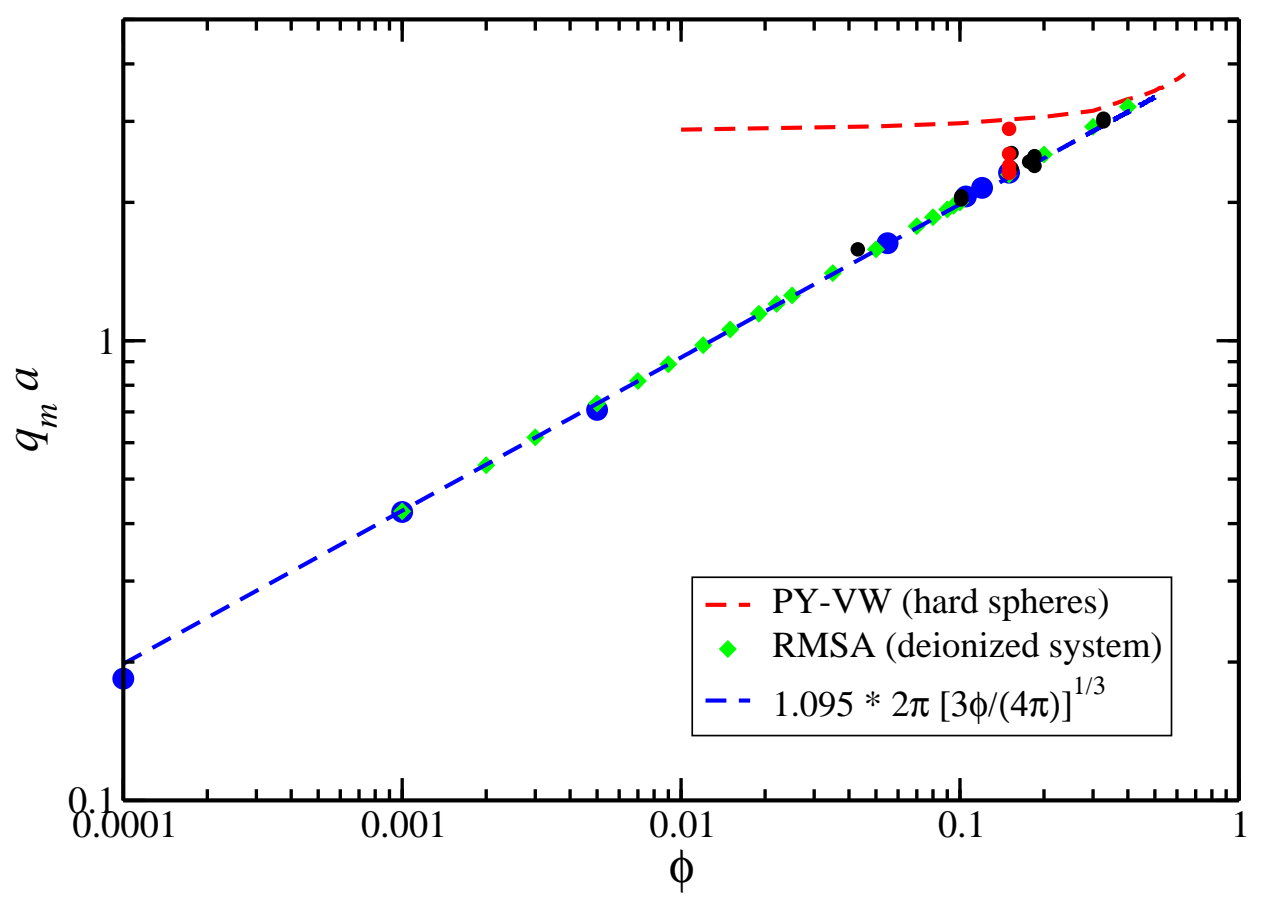


Banchio and Nägele - Fig. 6

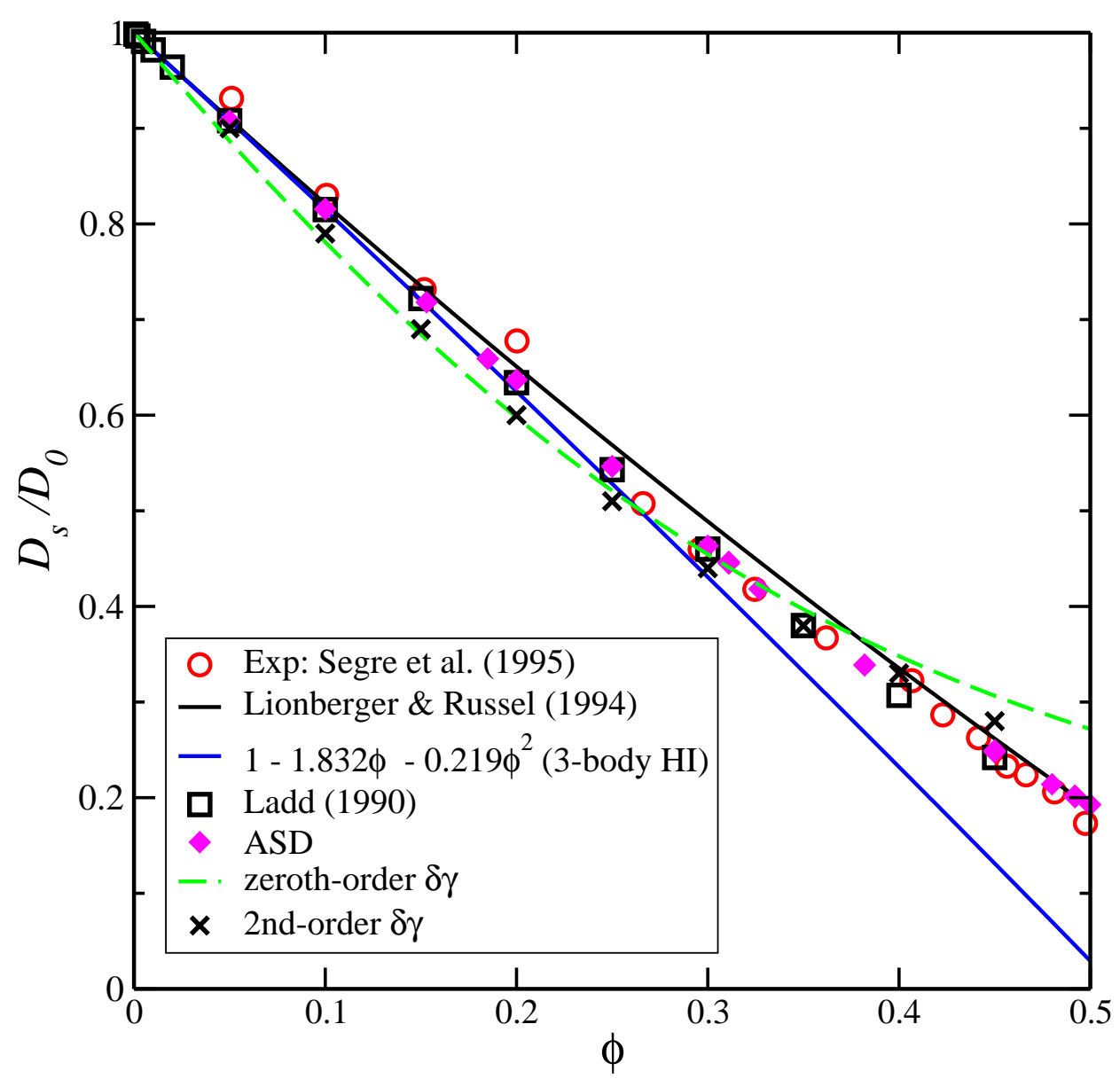


Banchio and Nägele - Fig. 7

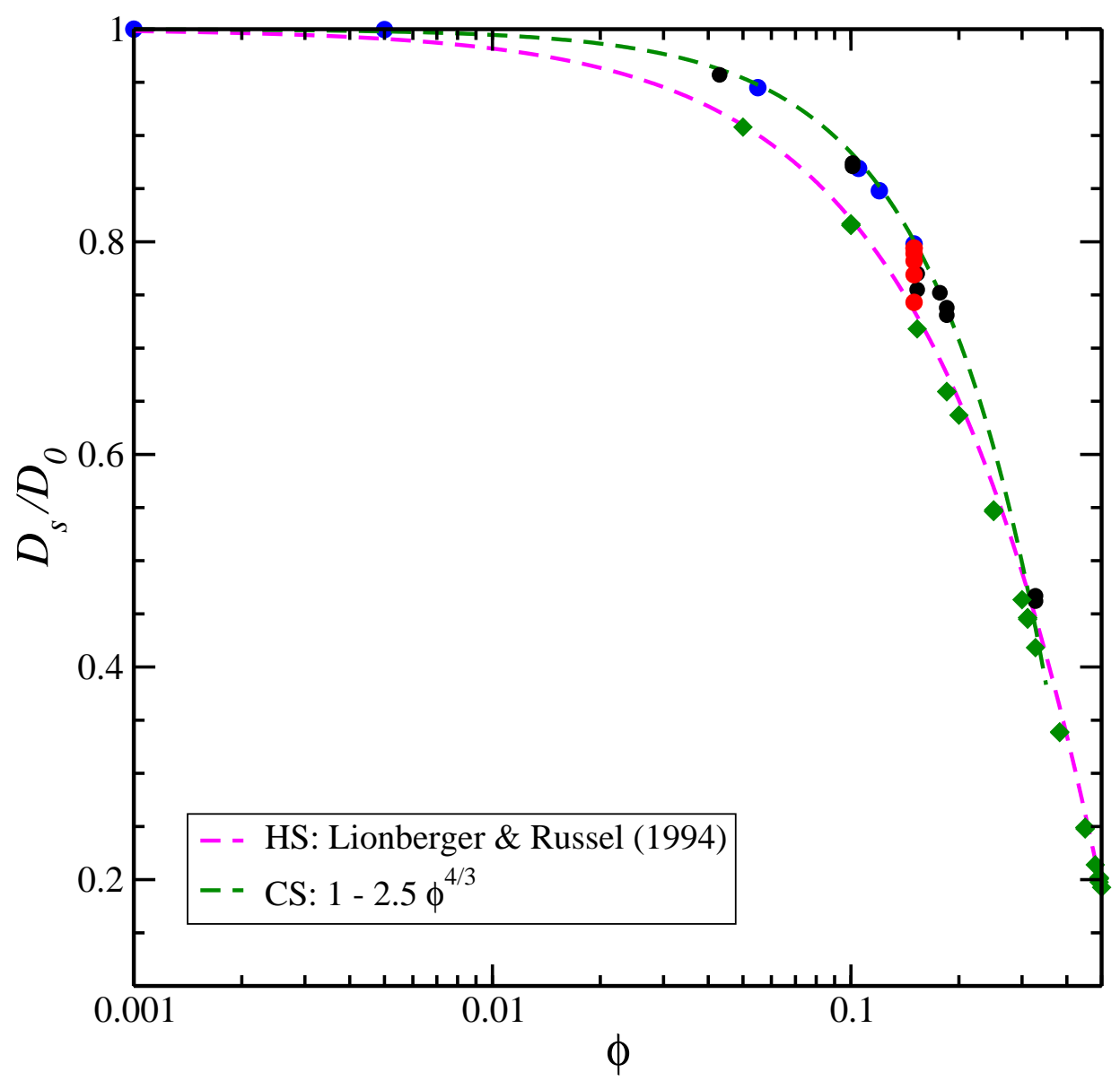


Banchio and Nägele - Fig. 8

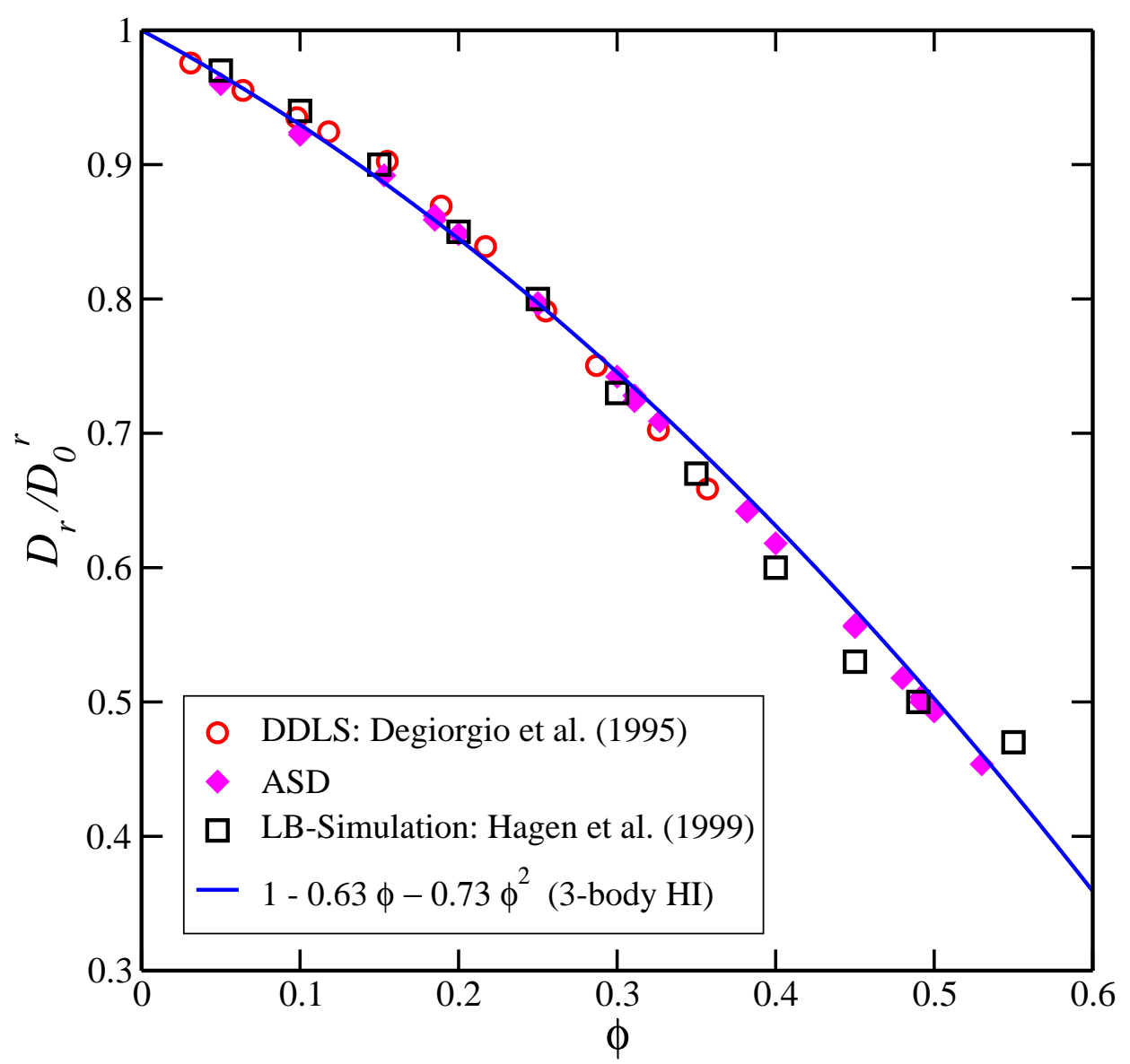


Banchio and Nägele - Fig. 9

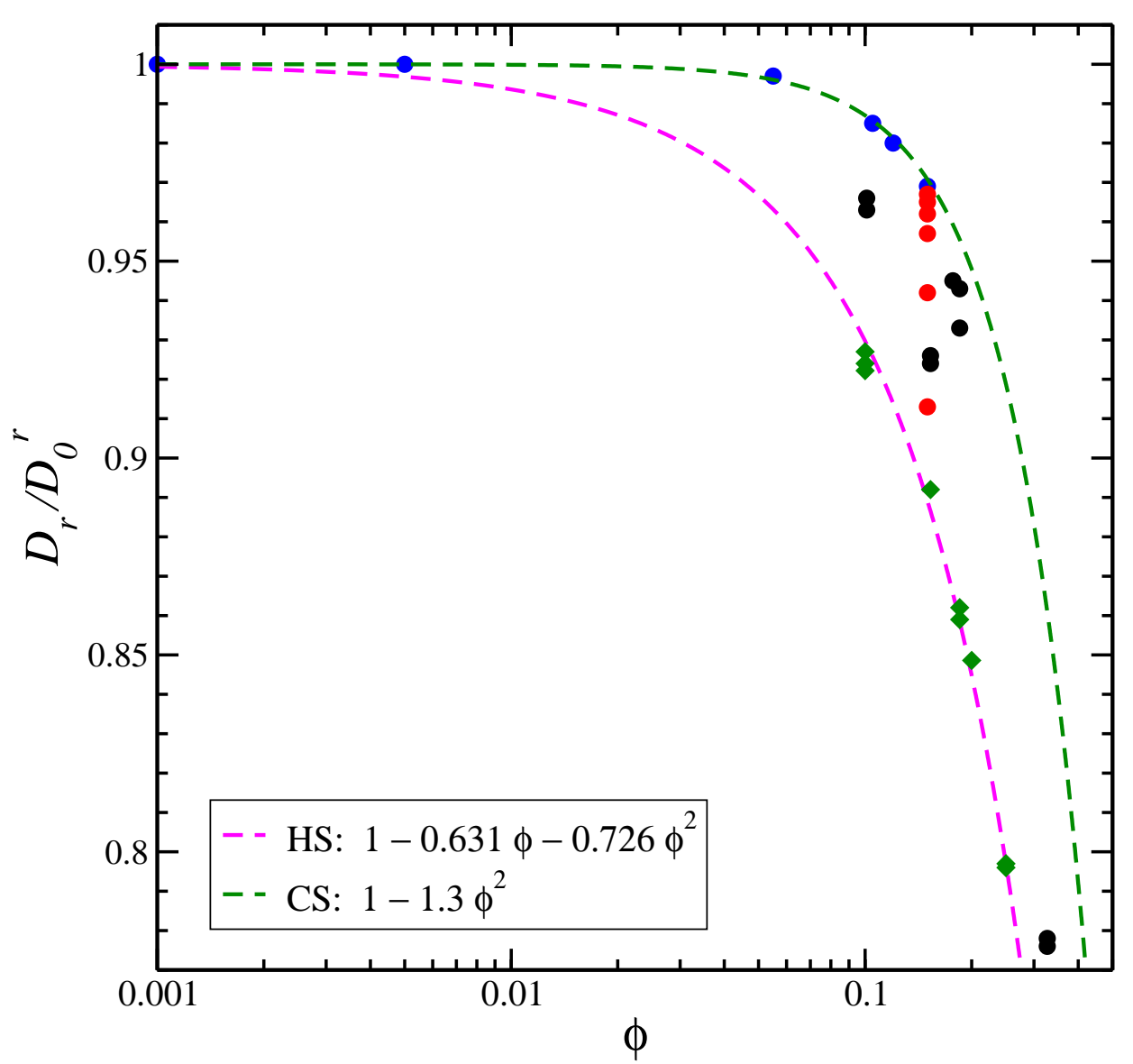


Banchio and Nägele - Fig. 10

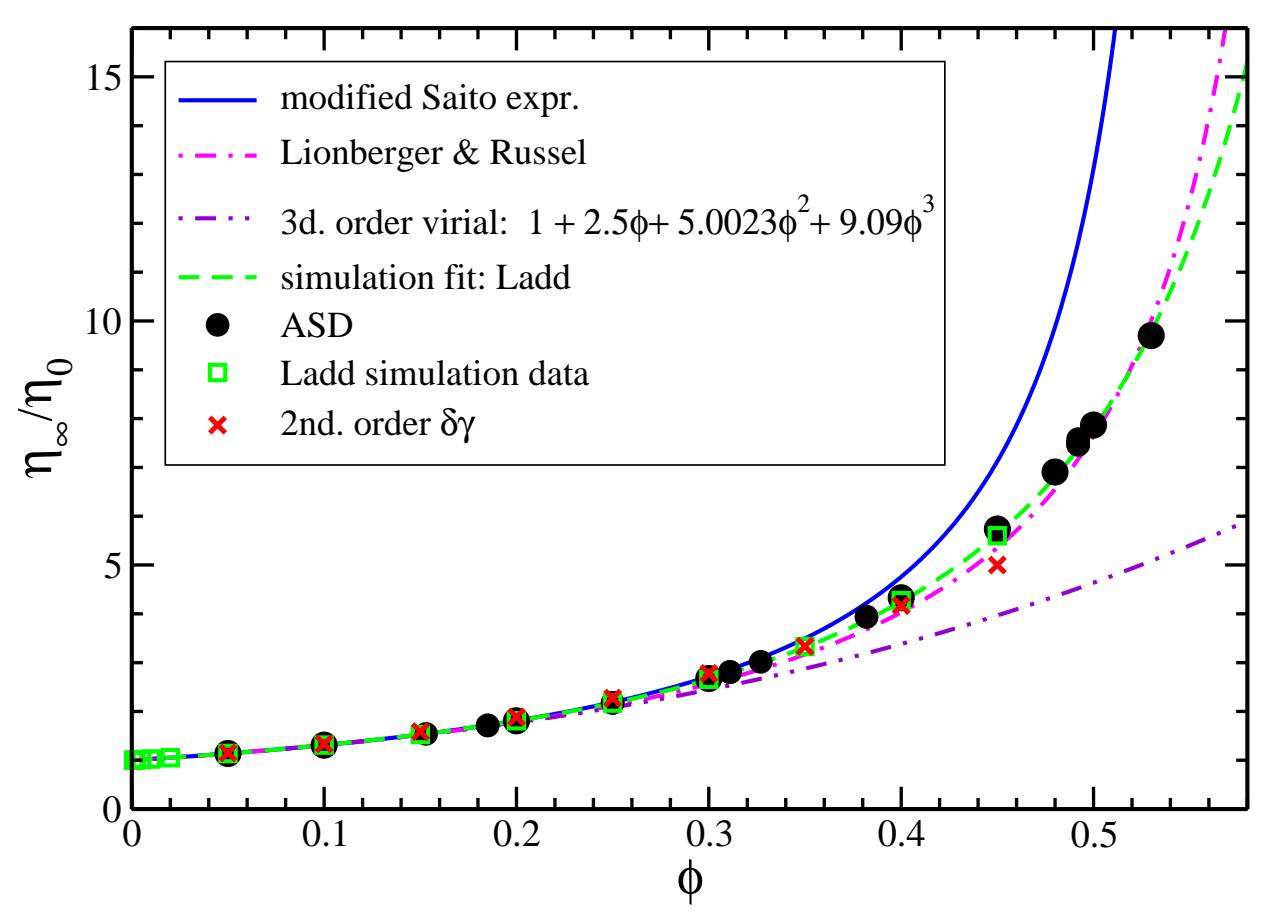


Banchio and Nägele - Fig. 11

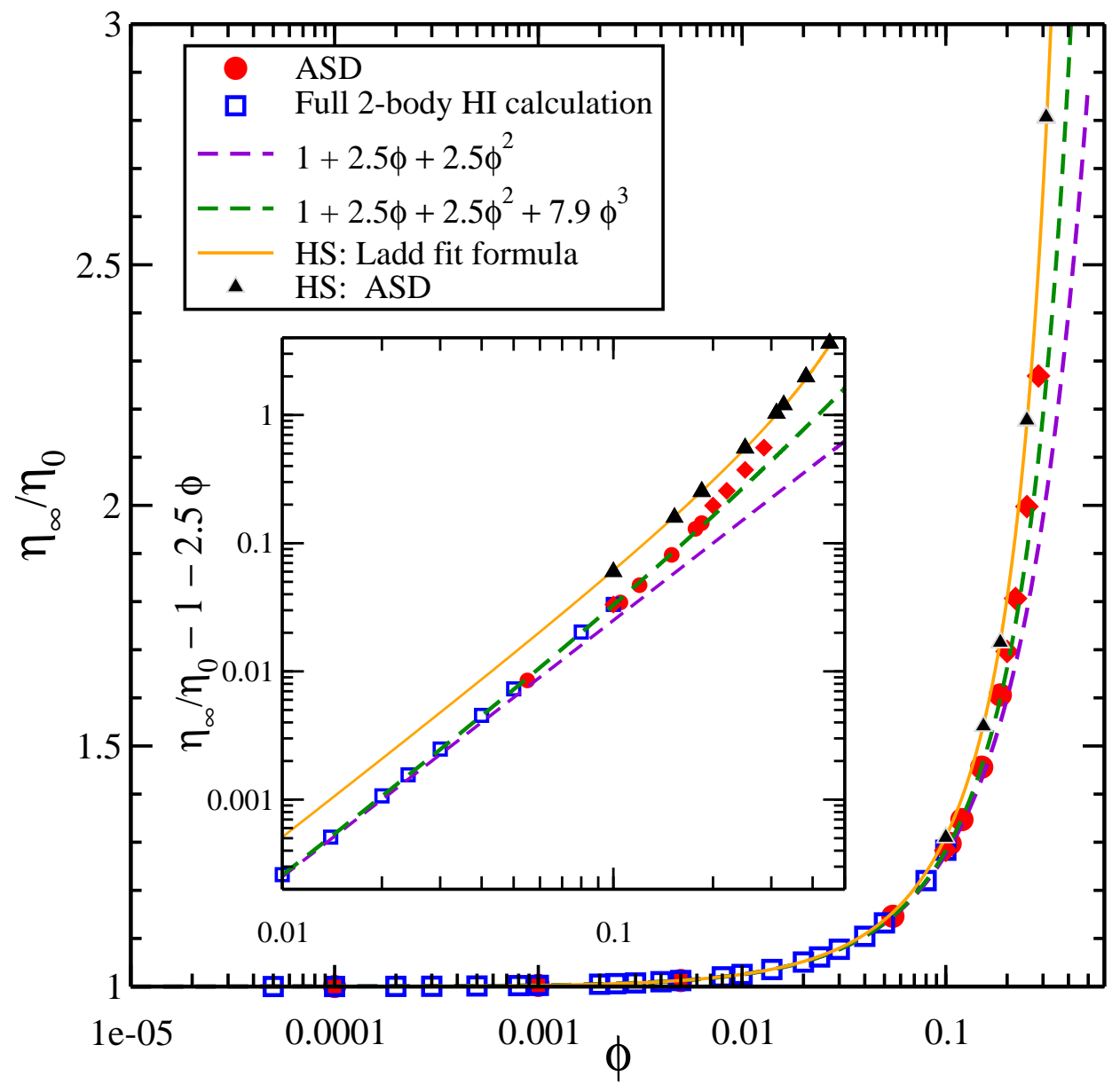




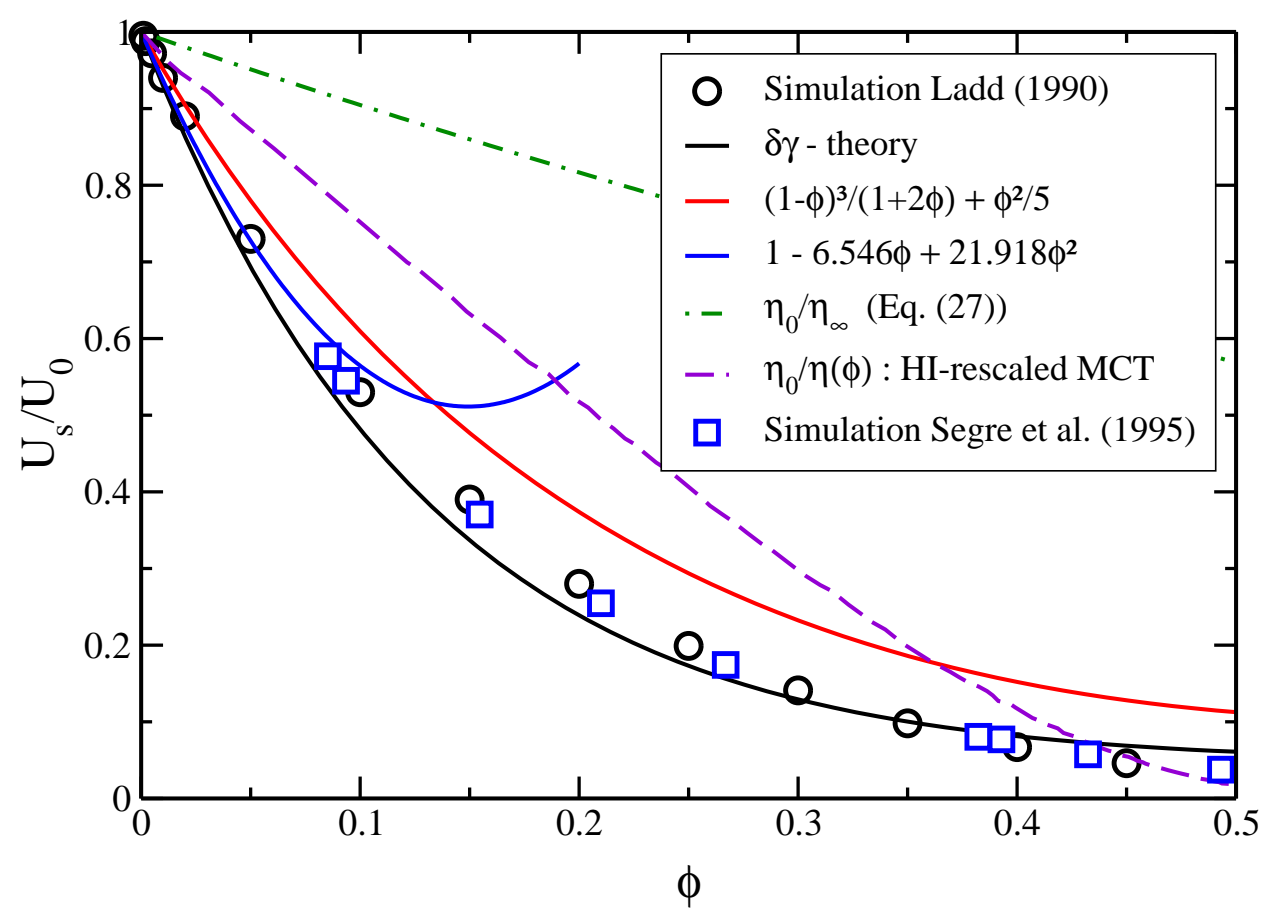


Banchio and Nägele - Fig. 13

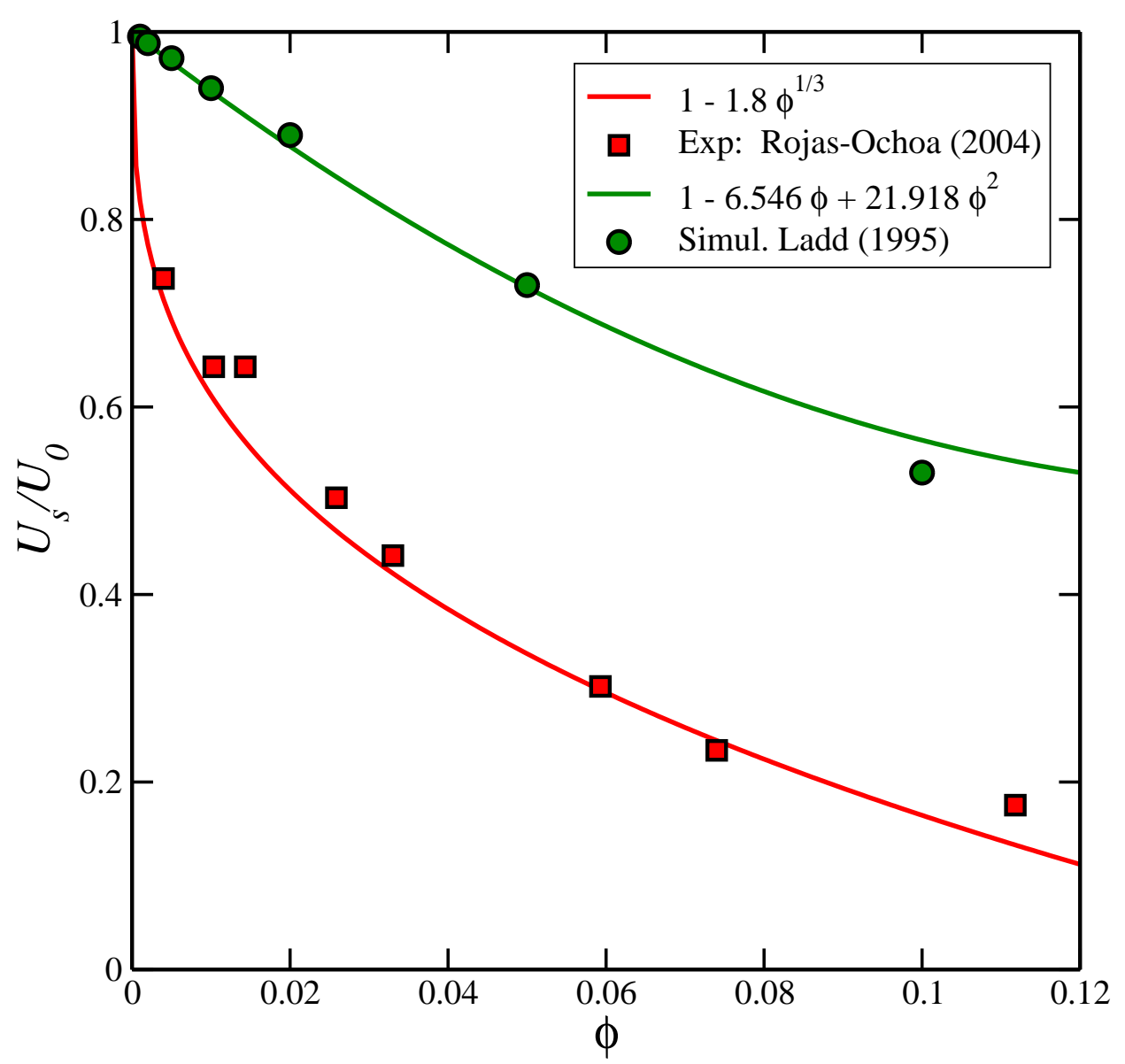


Banchio and Nägele - Fig. 14

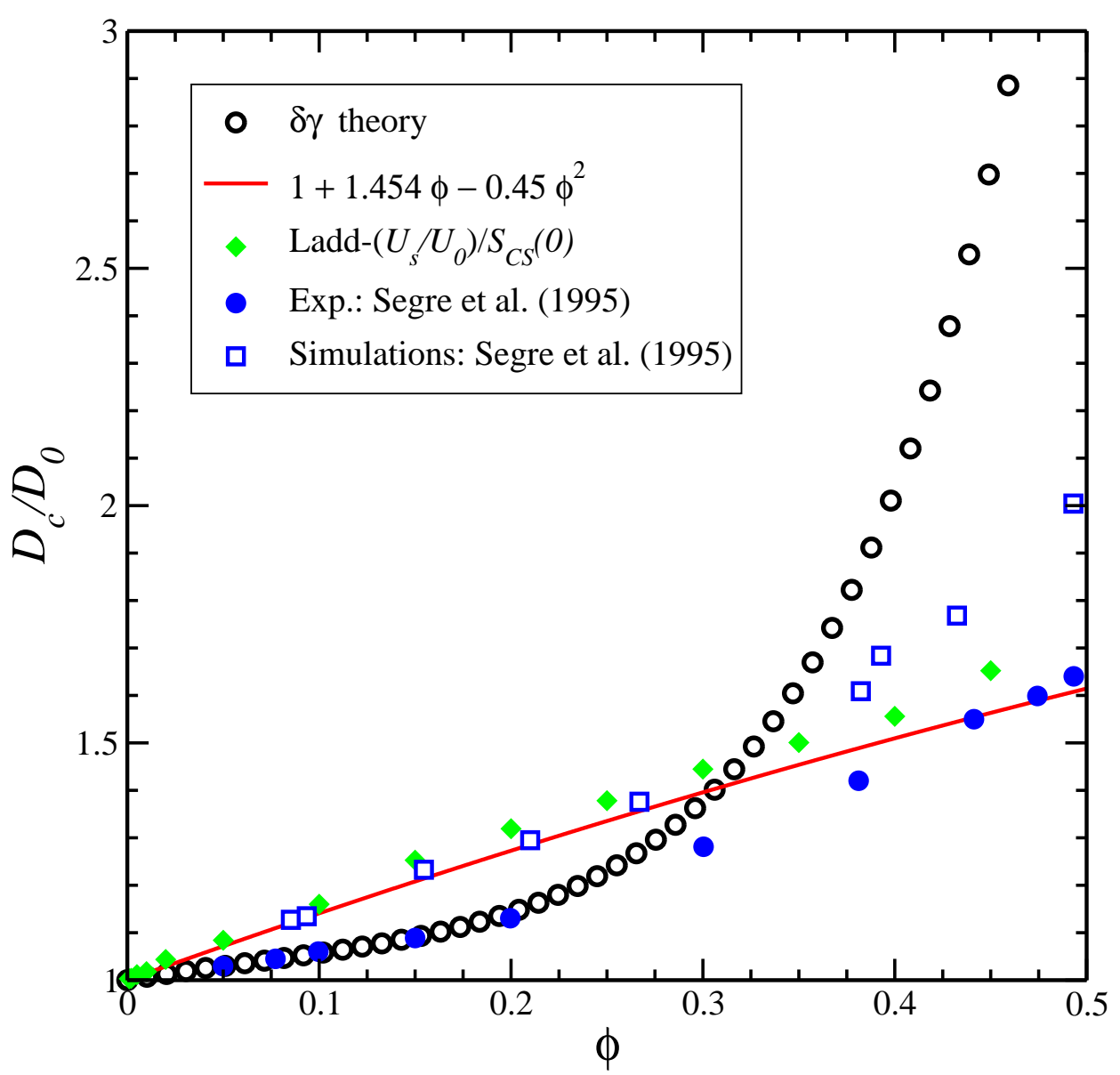


Banchio and Nägele - Fig. 15

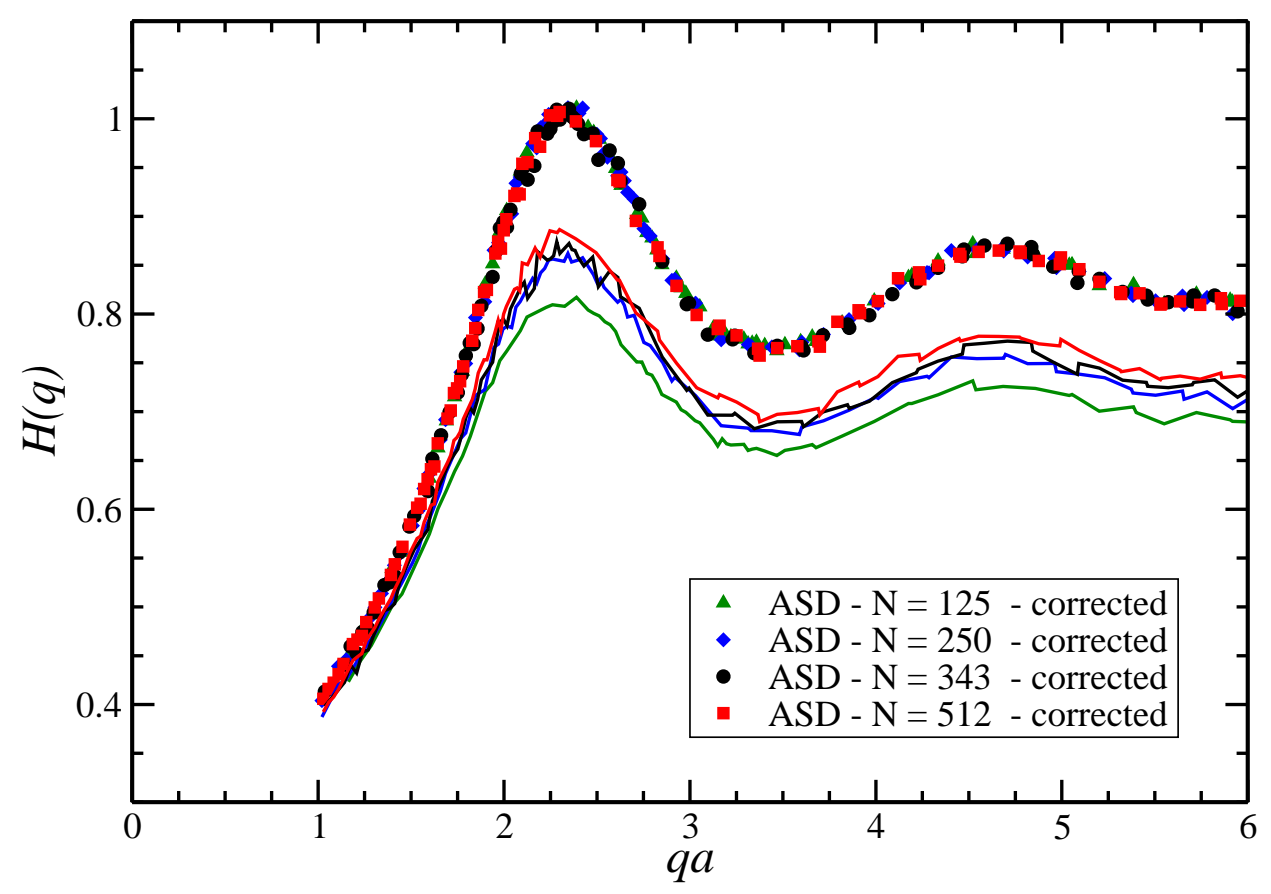


Banchio and Nägele - Fig. 16

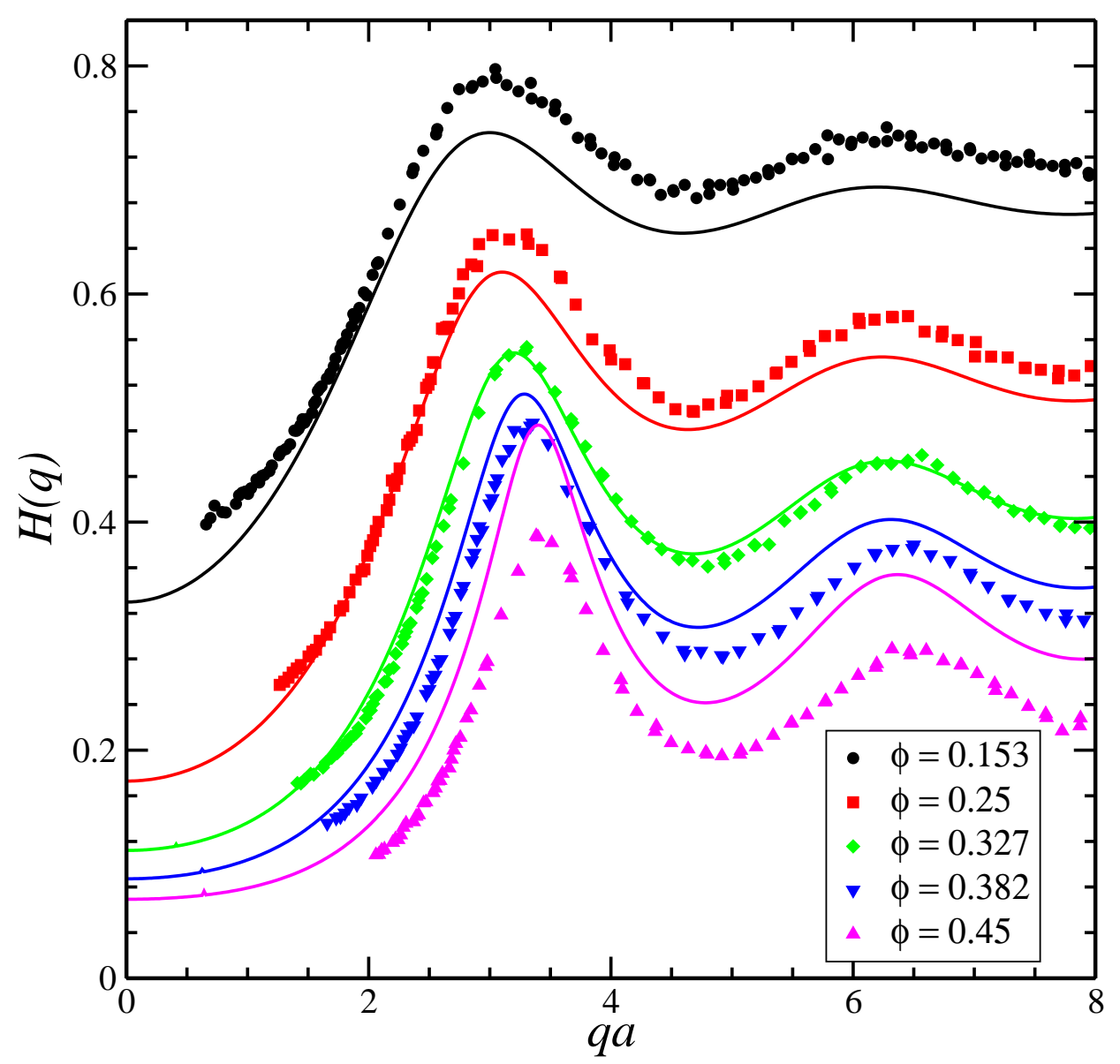


Banchio and Nägele - Fig. 17

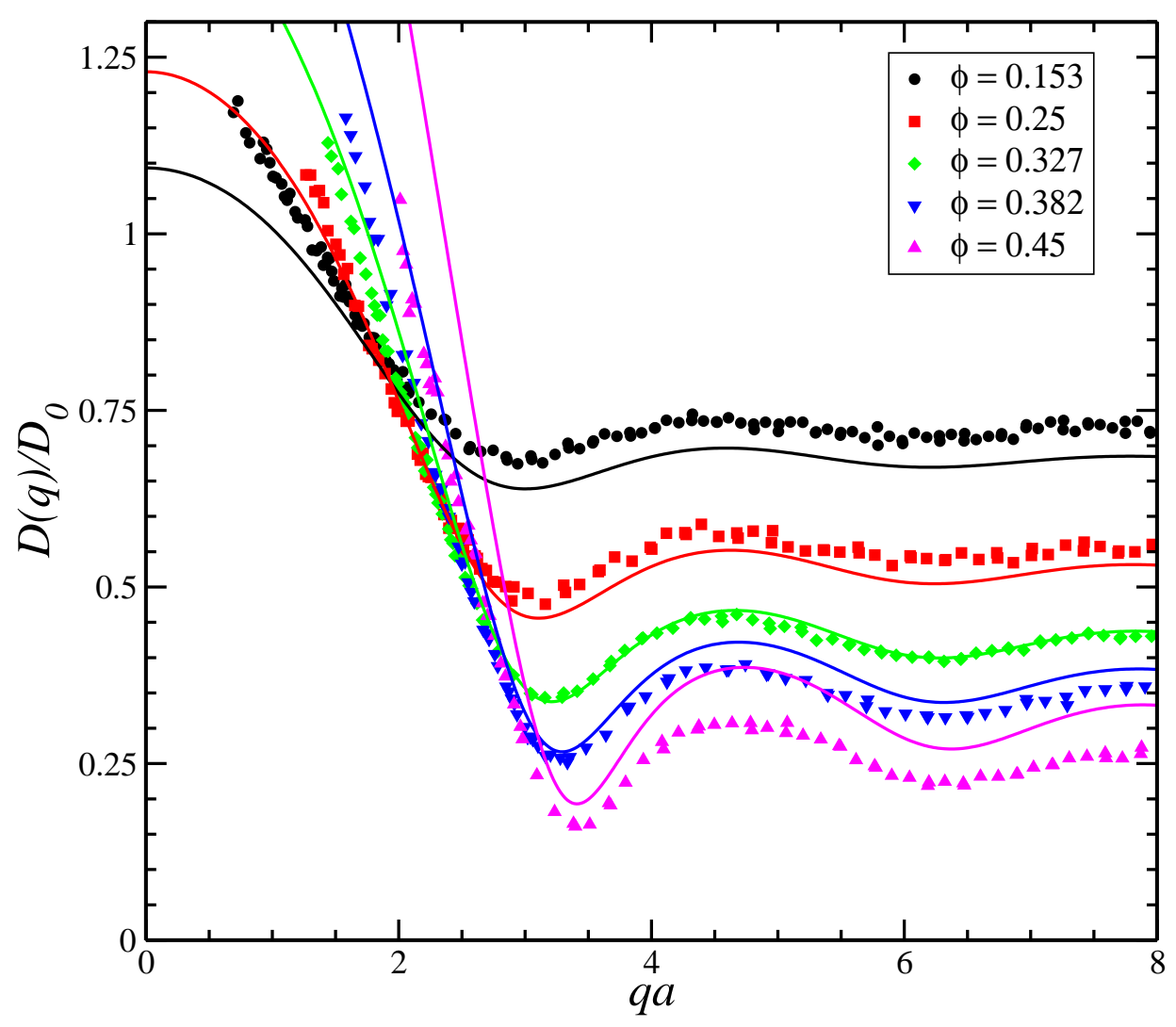


Banchio and Nägele - Fig. 18

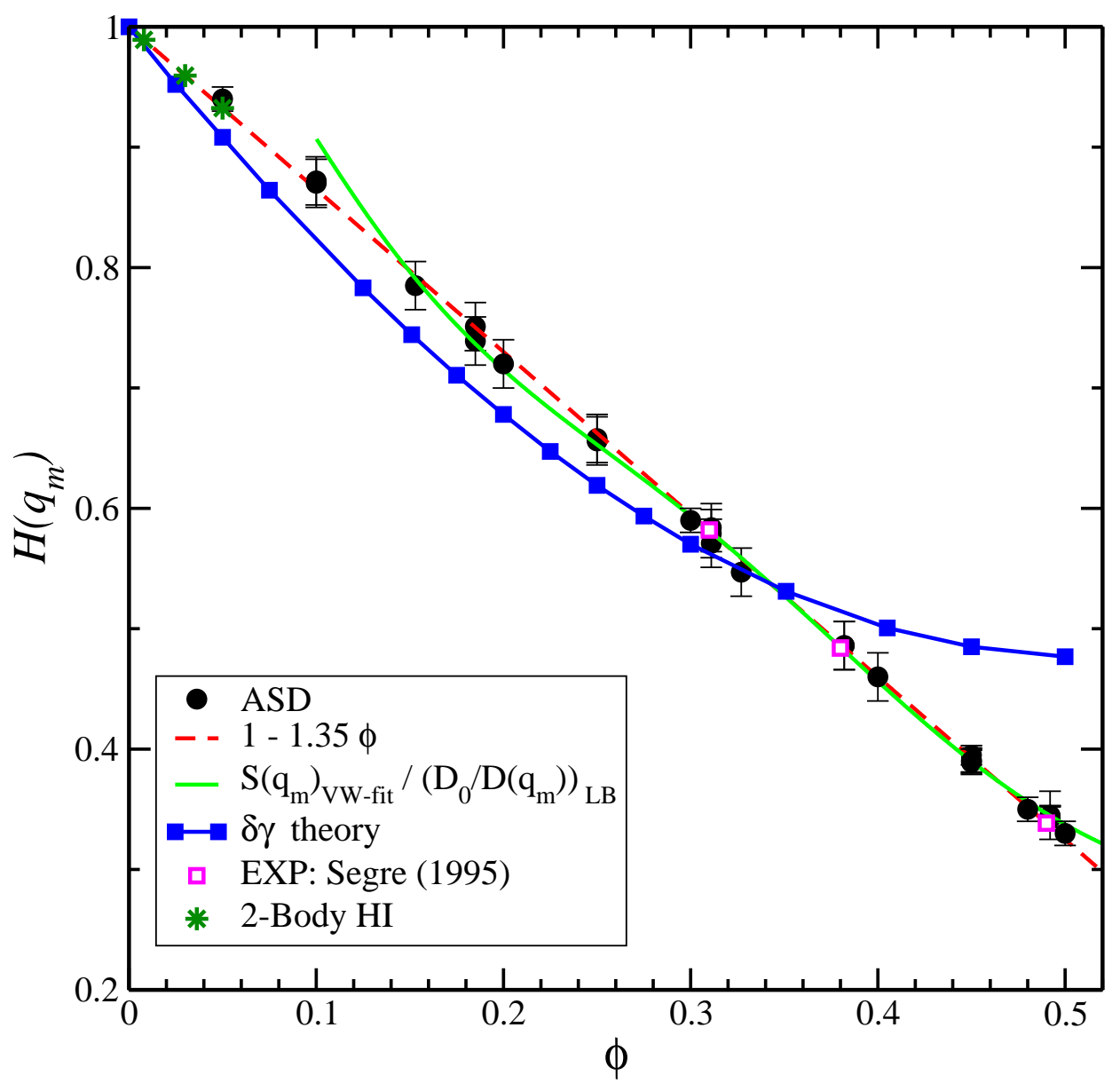


Banchio and Nägele - Fig. 19

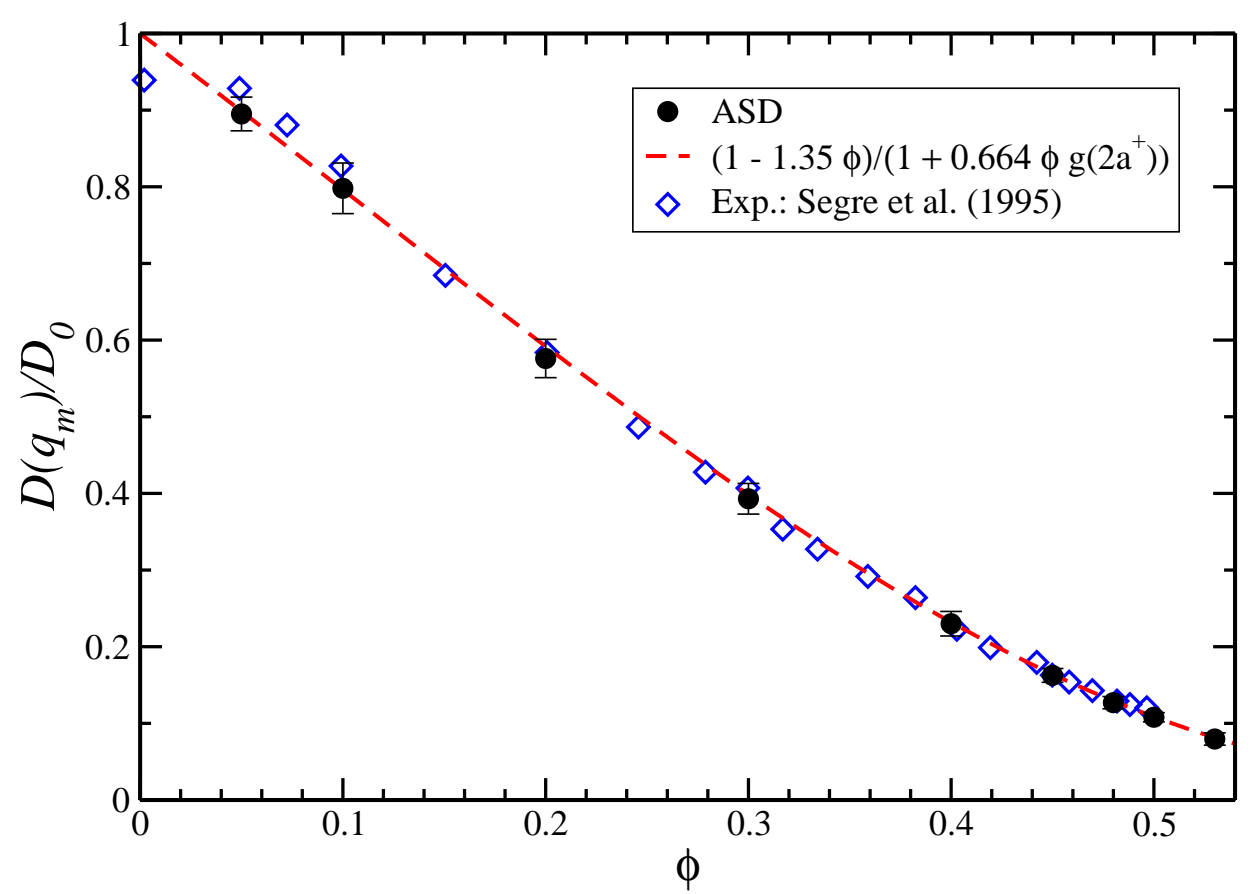


Banchio and Nägele - Fig. 20

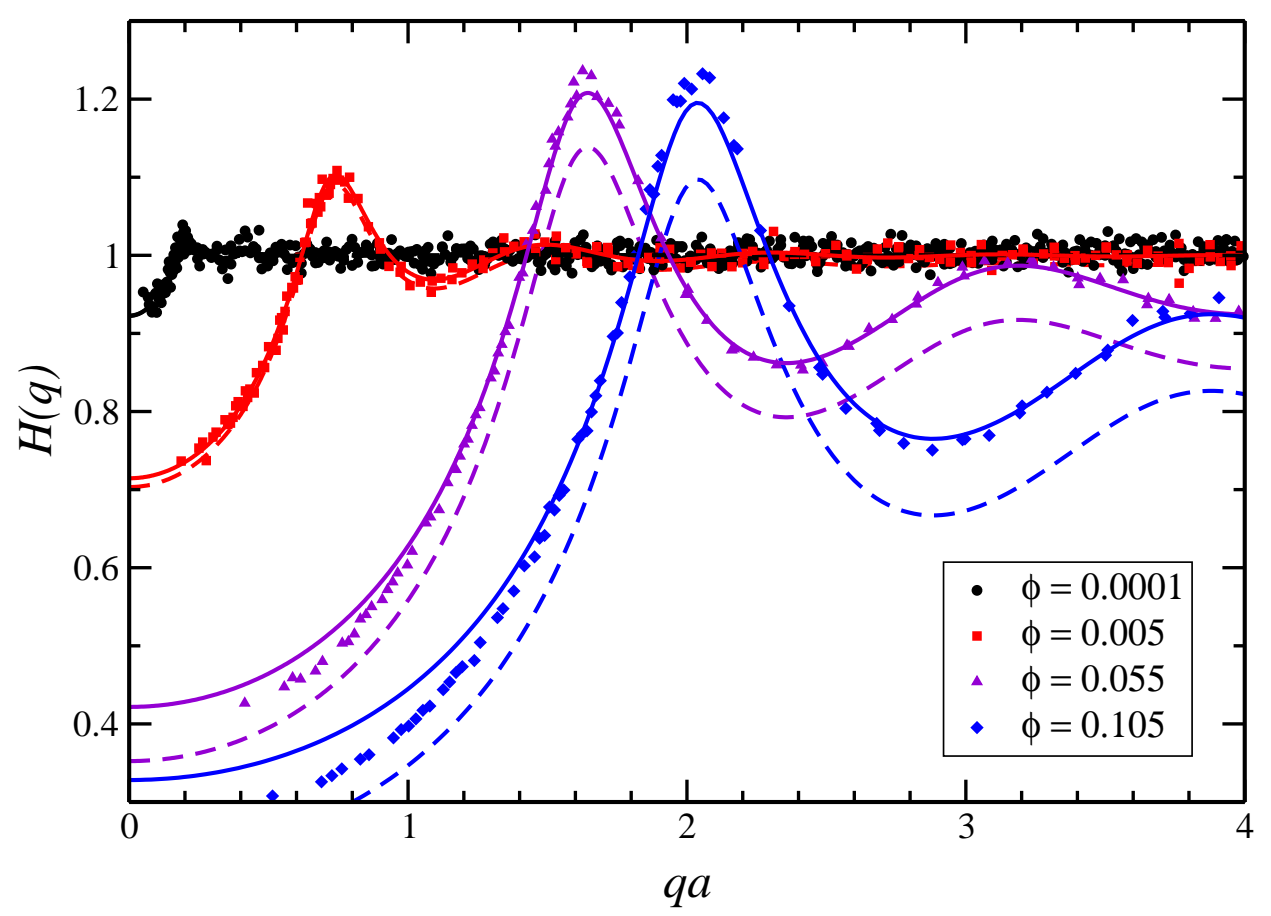




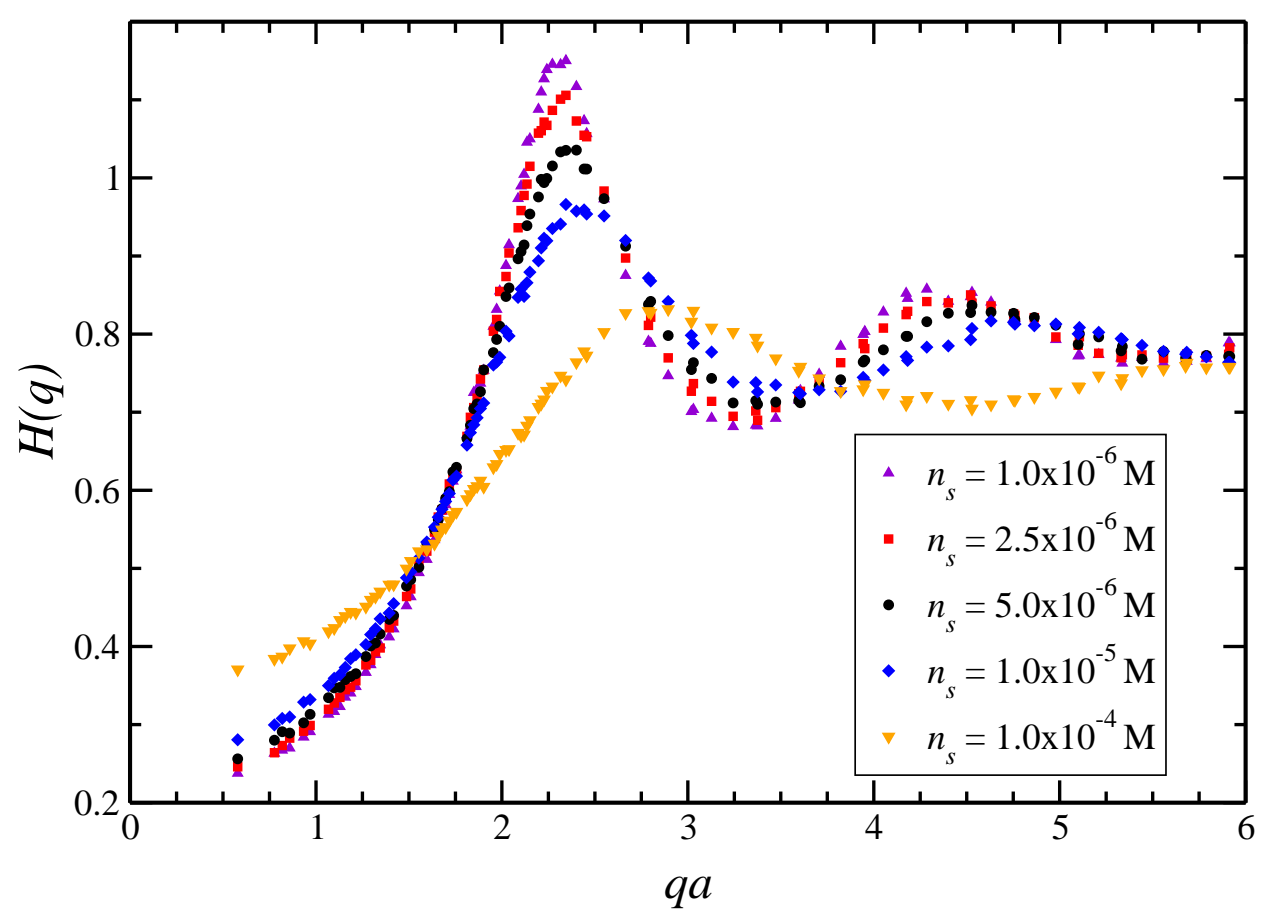


Banchio and Nägele - Fig. 22

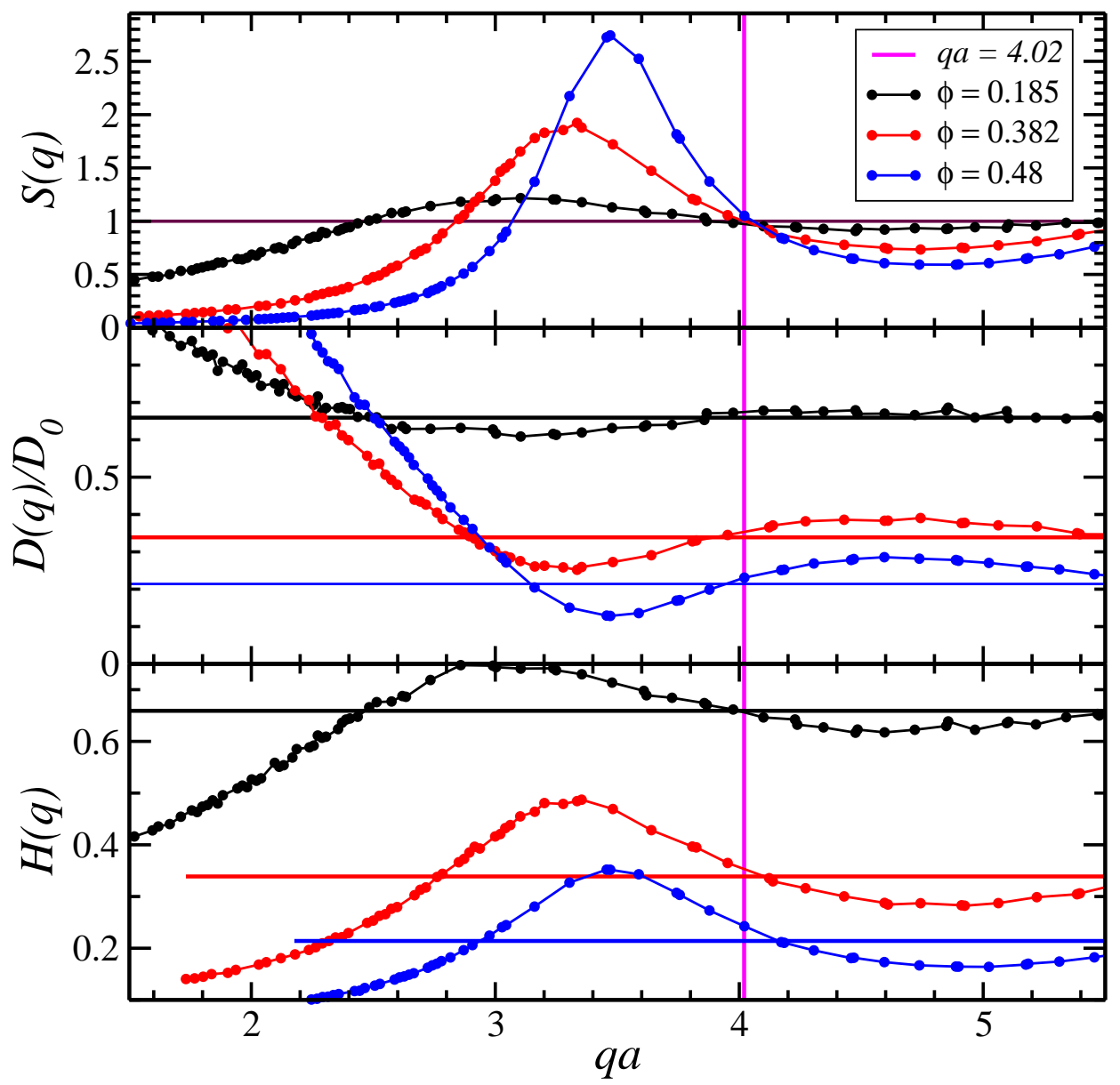


Banchio and Nägele - Fig. 23

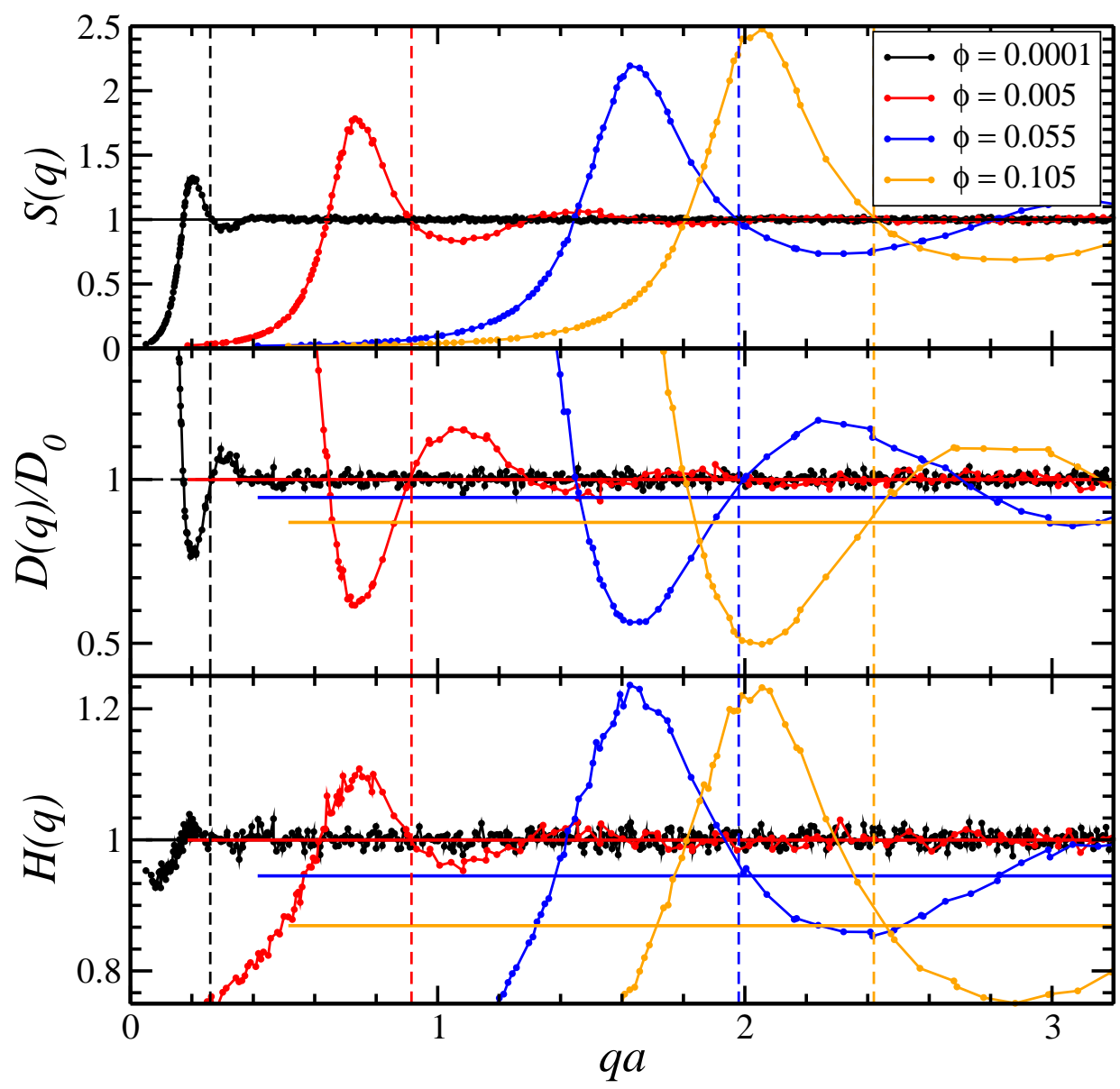


Banchio and Nägele - Fig. 24

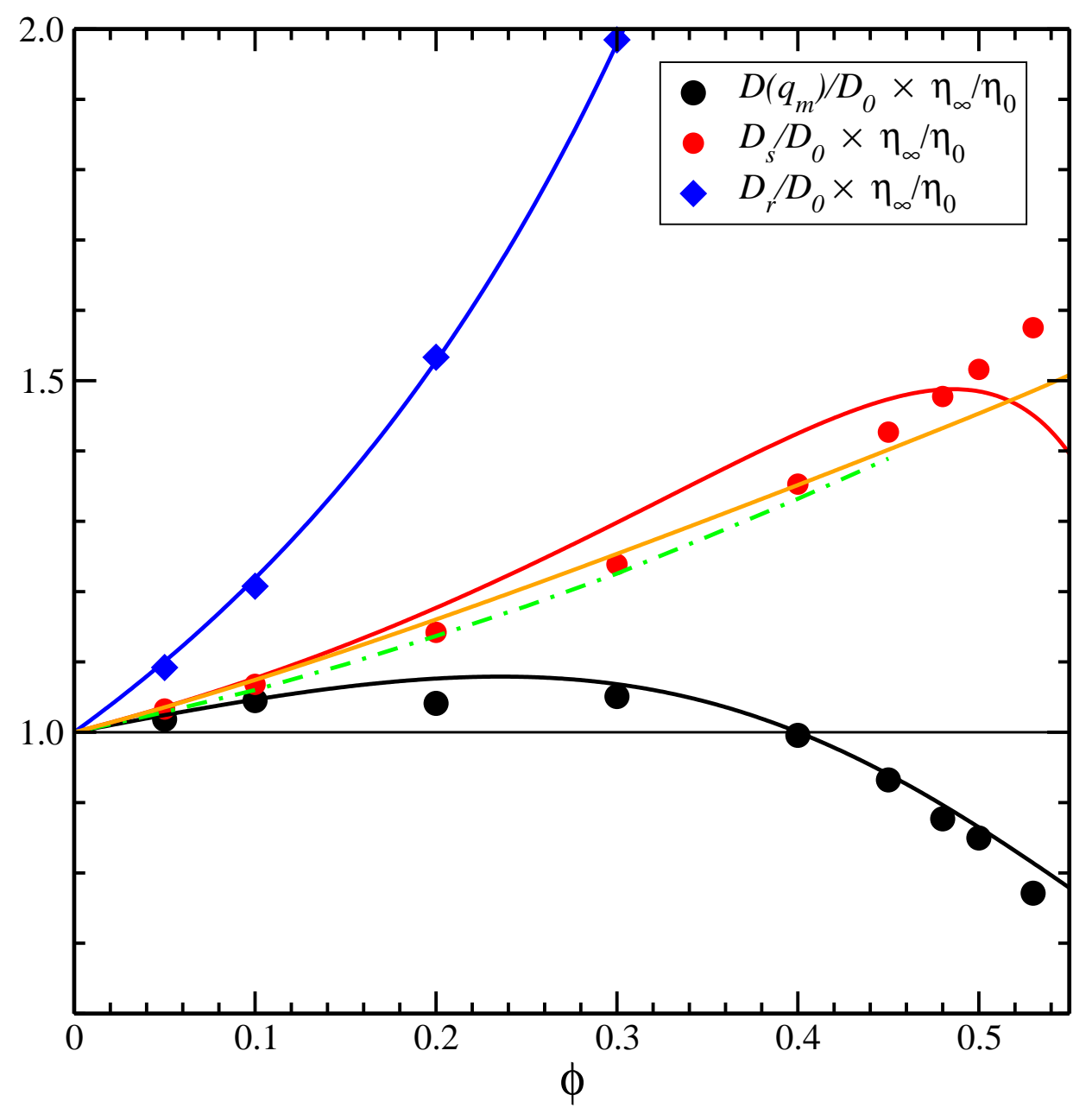


Banchio and Nägele - Fig. 25

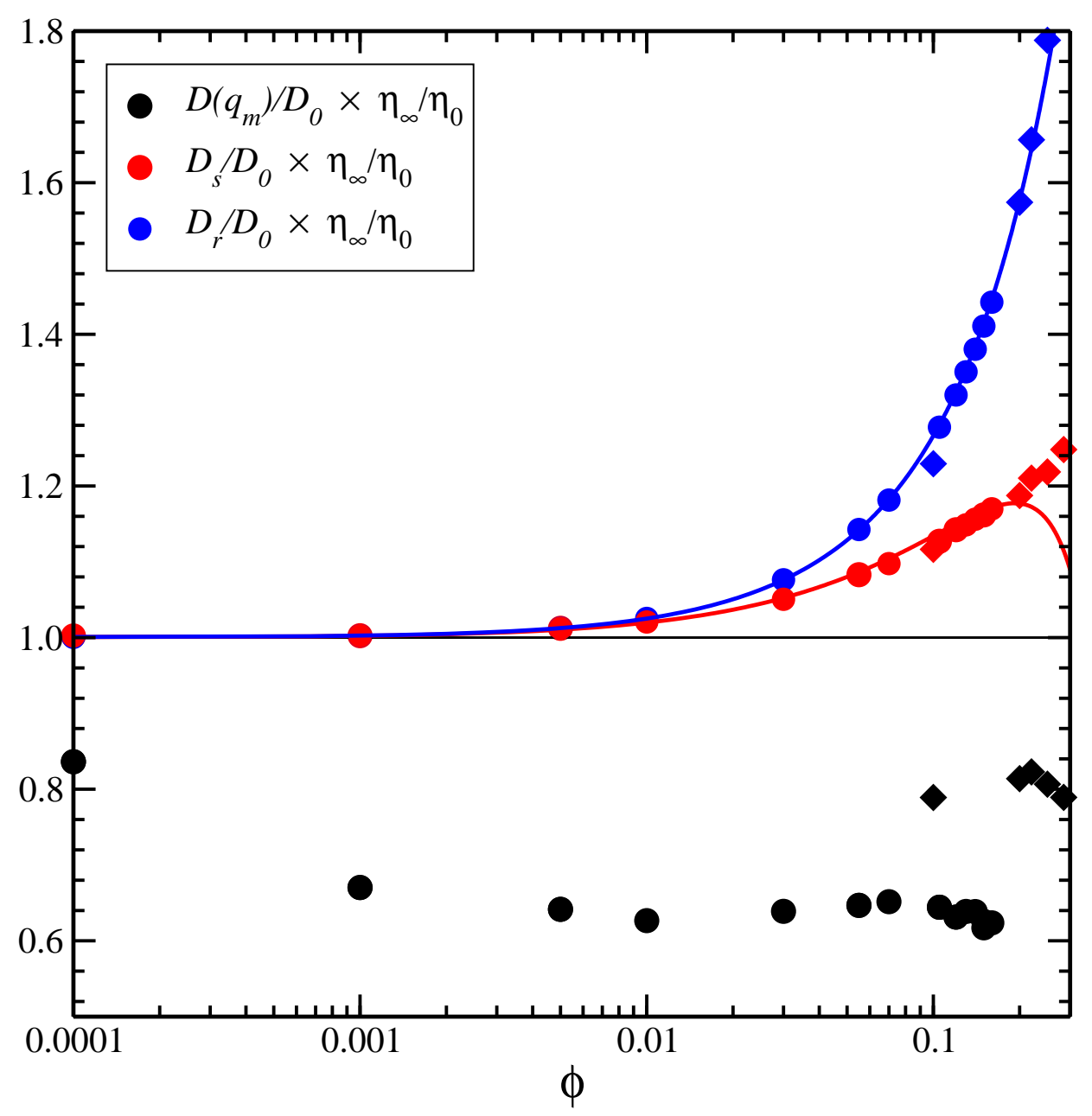

